Universidade de São Paulo

Instituto de Física

\title{
Correções da Massa do Alvo nas Funções de Estrutura
}

\author{
Samuel Mendes Sanches Júnior
}

Orientador: Prof. Dr. Fernando Silveira Navarra

\begin{abstract}
Dissertação de mestrado apresentada ao Instituto de Física para a obtenção do título de Mestre em Ciências.
\end{abstract}

Comissão Examinadora:

Prof. Dr. Fernando Silveira Navarra (orientador) (IFUSP)

Prof. Dr. Gastão Inácio Krein (IFTUNESP)

Prof. Dr. Renato Higa (IFUSP)

São Paulo 
FICHA CATALOGRÁFICA

Preparada pelo Serviço de Biblioteca e Informação do Instituto de Física da Universidade de São Paulo

Sanches Junior, Samuel Mendes

Correções da Massa do Alvo nas Funções de Estrutura. São Paulo, 2013.

Dissertação (Mestrado) - Universidade de São Paulo.

Instituto de Física. Depto. Física Experimental

Orientador: Prof. Dr. Fernando Silveira Navarra

Área de Concentração: Física

Unitermos: 1. Física de Partículas; 2. Fenomenologia;

3. Estrutura Nucleon.

USP/IF/SBI-088/2013 
Esta dissertação é dedicada ao meu amigo Tobby. Você foi e sempre será meu melhor amigo, muito obrigado por tudo. Durante 13 anos e 6 meses foi meu companheiro e me fez sentir que nunca estava sozinho. Mesmo sem dizer nenhuma palavra tornou minha vida muito mais feliz e alegre, sinto sua falta todos os dias. Descanse em paz.

À Tobby (in memoriam). 


\section{Agradecimentos}

A melhor parte de se terminar uma jornada é dar o respectivo agradecimento para quem nos ajudou. Assim, o primeiro e maior agradecimento são para meus pais, sempre me incentivando a seguir meus sonhos e apoiando emocionalmente e financeiramente, Samuel e Cleuri obrigado por simplesmente serem quem são. Ao meu irmão Alexandre e sua família, por sempre estarem do nosso lado e nos extensos almoços de domingo.

Em especial a minha querida namorada Suzi Diniz, pelo amor, amizade, risadas, dedicação e por ainda continuar de mãos dadas comigo nesse caminho tortuoso e difícil que chamamos de vida.

A professora Fernanda Steffens pelo tempo e orientação.

Ao professor Fernando Navarra, que sem sua ajuda não teríamos começado este trabalho.

Aos amigos que sempre estiveram comigo, em particular ao Alexis, Caio, Pedro e Vinicius, por mais de 10 anos de amizade e todas as cervejas.

Aos integrantes do GRHAFITE, ressaltando os amigos André, Bruno, David e Jorgivan, por fazer o dia a dia no instituto muito mais engraçado e divertido.

Ao café que certamente foi muito estimulante e presente durante este trabalho.

Ao bom e velho rock 'n' roll que é tema musical de minha vida e aos ilustres vídeos do Canal Away e Porta dos Fundos que em momentos difíceis e estressantes me fizeram gargalhar e conseguir continuar o trabalho.

Por fim, agradeço a CAPES pelo apoio financeiro. 


\section{Resumo}

Neste trabalho estudamos a estrutura do nucleon para grande $x$ de Bjorken e pequeno $Q^{2}$. Nesta região é preciso considerar correções nas funções de estrutura, de origem cinemática e dinâmica. Nos concentramos nas correções de origem cinemática, para as funções de estrutura não polarizadas, que surgem devido a massa do nucleon $\left(m_{p}\right)$ ser finita, essa correção é comumente chamada de Correções da Massa do Alvo (TMC). Revisamos a abordagem tradicionalmente utilizada e fizemos uma análise do problema inerente que se tem ao utilizar este tratamento (inclusão de regiões não físicas $x>1$ ), verificando a existência de uma inconsistência nesse método. Nossa solução para evitarmos este problema foi realizar uma expansão nas funções de estrutura com TMC ordem por ordem em $m_{p}^{2} / Q^{2}$, estudando numericamente a convergência da série obtida. Desta forma evitamos o problema da abordagem tradicional. 


\section{Abstract}

In this work we study the nucleon structure for large Bjorken $x$ and low $Q^{2}$. In such region is necessary to consider corrections on the structure functions, with by using kinematics and dynamics origins. We focus our study in the corrections of kinematic origin, for non-polarized structure functions, which cames from the finite nucleon mass $\left(m_{p}\right)$ and it is generally known by Target Mass Corrections (TMC). We review the traditional approach and perform an analysis of the inherent problem that has been in this treatment (inclusion of non-physical regions $x>1$ ), which provides the inconsistency of this method. Our solution to avoid this problem was to perform an expansion order by order in $m_{p}^{2} / Q^{2}$ on the structure functions with TMC and perform the numerical study of the convergence of the series obtained. By this way we avoid the problem of the traditional approach. 


\section{Conteúdo}

Introdução 1

1 Estrutura Interna do Nucleon 5

1.1 Espalhamento elétron-múon . . . . . . . . . . . . . . . 5

1.2 Espalhamento elétron-próton . . . . . . . . . . . . . . . . . . . 8

1.2.1 Espalhamento elástico . . . . . . . . . . . . . . . . 8

1.2.2 Espalhamento inelástico . . . . . . . . . . . . . . . 12

1.3 Modelo de pártons e o scaling de Bjorken . . . . . . . . . . . . . 15

2 Expansão do Produto de Operadores 23

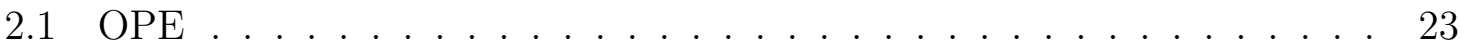

2.2 Momento das funções de estrutura . . . . . . . . . . . . . . . . . . . 27

3 Correções da Massa do Alvo pela OPE 36

3.1 Abordagem utilizada por Georgi e Politzer . . . . . . . . . . . . . 37

3.1.1 Momento de Cornwall-Norton com as TMC . . . . . . . . . . . 38

3.1.2 Funções de estrutura com as TMC . . . . . . . . . . . . . . . . 43

3.2 Momento de Nachtmann . . . . . . . . . . . . . . . . . . . . . . . 48

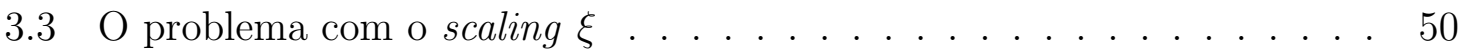

3.3.1 Consistência na TMC com o scaling $\xi \ldots . . . . . . . . ~ 53$

4 Correções da Massa do Alvo como Expansão em Série $\quad 57$

4.1 TMC como inversão termo por termo . . . . . . . . . . . . . 57 
4.2 Convergência das séries . . . . . . . . . . . . . . . . . . 62

5 Conclusões $\quad 69$

A Funções de Estrutura com Abordagem de Georgi e Politzer $\quad 72$

A.1 $F_{1}^{T M C}\left(x, Q^{2}\right)$, Georgi e Politzer . . . . . . . . . . . . 72

A.2 $F_{L}^{T M C}\left(x, Q^{2}\right)$, Georgi e Politzer . . . . . . . . . . . . 76

A.3 $F_{3}^{T M C}\left(x, Q^{2}\right)$, Georgi e Politzer . . . . . . . . . . . . . 77

A.4 $F_{4}^{T M C}\left(x, Q^{2}\right)$, Georgi e Politzer . . . . . . . . . . . . . 80

A.5 $F_{5}^{T M C}\left(x, Q^{2}\right)$, Georgi e Politzer ................ 84

B Funções de Estrutura com Inversão Termo a Termo 88

B.1 $F_{1}^{T M C}\left(x, Q^{2}\right)$, série em $\mu \ldots \ldots \ldots \ldots$

B.1.1 Convergência da série para $F_{1}\left(x, Q^{2}\right) \ldots \ldots . \ldots 90$

B.2 $F_{L}^{T M C}\left(x, Q^{2}\right)$, série em $\mu \ldots \ldots \ldots \ldots$

B.3 $F_{3}^{T M C}\left(x, Q^{2}\right)$, série em $\mu \ldots \ldots \ldots \ldots \ldots \ldots \ldots$

B.3.1 Convergência da série para $F_{3}\left(x, Q^{2}\right) \ldots \ldots$. . . . . . . 94

B.4 $F_{4}^{T M C}\left(x, Q^{2}\right)$, série em $\mu \ldots \ldots \ldots \ldots$. . . . . . . . . . 97

B.5 $F_{5}^{T M C}\left(x, Q^{2}\right)$, série em $\mu \ldots \ldots \ldots \ldots . \ldots \ldots$

$\begin{array}{ll}\text { Referências Bibliográficas } & 100\end{array}$ 


\section{Lista de Figuras}

1.1 Diagrama de Feynman em ordem mais baixa para o espalhamento $e+$ $\mu \rightarrow e^{\prime}+\mu^{\prime} \ldots \ldots \ldots \ldots \ldots \ldots \ldots \ldots \ldots \ldots \ldots \ldots \ldots \ldots \ldots$

1.2 Diagrama de Feynman em ordem mais baixa para o espalhamento $e+$ $p \rightarrow e^{\prime}+p^{\prime}$, onde a bola no vértice $\gamma-p$ esquematiza os fatores de forma. 9

1.3 Fatores de forma de Sachs para o próton em função de $q^{2}$. Extraído de

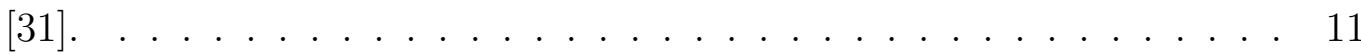

1.4 Seção de choque para $e+p \rightarrow e^{\prime}+X$ em função da massa do sistema hadrônico $X$ produzido, $W^{2}=(p+q)^{2}$. O pico elástico em $W=M$ foi reduzido por um fator de 8.5. Extraído de [31]. . . . . . . . . . . . 12

1.5 Diagrama de Feynman em ordem dominante do espalhamento $e+p \rightarrow$

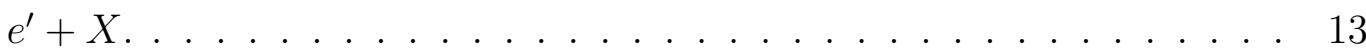

1.6 Diagrama de Feynman para o espalhamento Compton virtual. . . . . . 15

1.7 Comportamento de $2 m_{p} W_{1}$, não se nota variação significativa com $Q^{2}$. Extraído de [36]. . . . . . . . . . . . . . . . . . . . . 16

1.8 Comportamento de $\nu W_{2}$ para vários $\omega$, não se nota variação significativa com $Q^{2}$. Extraído de [36] . . . . . . . . . . . . . . . . 16

1.9 Evidência da quebra de scaling, os dados estão normalizados pelo valor em parêntesis. Extraído de [10]. . . . . . . . . . . . . . . 17

1.10 Interpretação do modelo de pártons para $e+p \rightarrow e^{\prime}+X, q_{i}$ representa o párton do tipo $i \ldots \ldots \ldots \ldots \ldots \ldots \ldots \ldots$

1.11 Teste da relação (1.33). Extraído de [43] . . . . . . . . . . . . . . 20 
1.12 Estrutura das PDFs com relação a $x$, geradas para $Q^{2}=100 \mathrm{GeV}^{2}$ com as parametrizações dada pela MSTW 2008 [16], na legenda $q_{i}=u_{i}+d_{i}+s_{i} .22$

2.1 Caminho $\mathcal{C}$ de integração usado em (2.17) . . . . . . . . . . . . . . 30

3.1 Variável de Nachtmann, equação (3.1), em função de $x$ para vários valores de $Q^{2}$ e o caso limite $\xi=x \ldots \ldots 37$

$3.2 \quad F_{2}$ com (linhas contínuas) e sem TMC (linhas tracejadas) para vários $Q^{2}$, sendo a PDF dada pela MSTW $2008[16]$. . . . . . . . . . . . . 46

3.3 Comparação da função de estrutura $F_{2}$ com (linhas contínuas) e sem TMC (linhas tracejadas), junto com dados do NuTeV [79], sendo a PDF dada pela CTEQ6HQ [80]. Extraído de [27]. . . . . . . . . . . . . 47

3.4 Comparação do momento de Cornwall-Norton (linha contínua) $\operatorname{com} n=$ 2 e sem TMC, com o momento de Nachtmann (linha tracejada) (3.31b) com $n=2$, usando a TMC (3.29) e com a mesma distribuição utilizada no momento de Cornwall-Norton. Extraído de [27]. . . . . . . . . . . . 50

$3.5 M_{2}^{(n=2)}\left(Q^{2}\right)$ (linhas coloridas), equação (3.17), para $j=0$ (linha pontilhada), $j<2$ (linha ponto-traço-traço), $j<3$ (linha ponto-traço) e $j<4$ (linha tracejada), comparada com a inversão (3.39), sendo o limite superior de integração $\xi_{\max }=\xi_{0}$ (linha vermelha ponto-ponto-traço) e $\xi_{\max }=1$ (linha vermelha contínua). Extraído de [78] . . . . . . . . 56

4.1 TMC para $F_{2}$ em $Q^{2}=1 G e V^{2}$ pela equação (4.8), mostrando a convergência com o aumento de $j$, comparada com o resultado da equação (3.29), em função de $x$. . . . . . . . . . . . . . . . . .

4.2 O mesmo que a Figura (4.1), mas em função de $W$ (massa do estado hadrônico final). . . . . . . . . . . . . . . . . . . .

4.3 Razão entre a TMC $F_{2} \operatorname{com} Q^{2}=1 \mathrm{GeV}^{2}$ dada por (4.8) para vários $j$ $(j=0$ até $j<5)$ com a TMC (3.29), em função de $x$ (acima) e de $W$ (abaixo). . . . . . . . . . . . . . . . . 65 
4.4 Razão entre a TMC $F_{2} \operatorname{com} Q^{2}=5 G e V^{2}$ dada por (4.8) para vários $j$ $(j=0$ até $j<5)$ com a TMC (3.29), em função de $x$ (acima) e de $W$

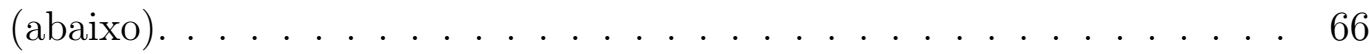

4.5 TMC para $F_{2}$ em $Q^{2}=2 G e V^{2}$ pela equação (4.8), mostrando a convergência para grandes valores de $j<101,102,201,202,301$ e 302, em

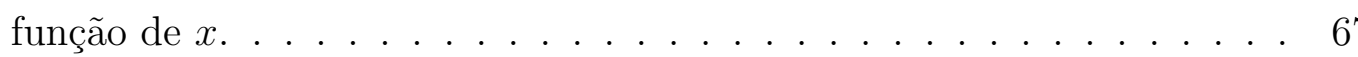

4.6 Razão entre a TMC $F_{2} \operatorname{com} Q^{2}=2 G e V^{2}$ dada por (4.8) para vários $j$ com a TMC (3.29), em função de $x$ (esquerda) e de $W$ (direita). . . . . 67

4.7 TMC para $F_{2}$ em $Q^{2}=2 \mathrm{GeV}^{2}$ (acima) e $Q^{2}=5 \mathrm{GeV}^{2}$ (abaixo) pela equação (4.8), mostrando a convergência com o aumento de $j$, comparada com o resultado da equação (3.29), em função de $x$ (esquerda) e $W$ (direita). . . . . . . . . . . . . . . . . 68

B.1 TMC para $F_{1}$ em $Q^{2}=1 G e V^{2}$ (acima), $Q^{2}=2 G e V^{2}$ (meio) e $Q^{2}=$ $5 \mathrm{GeV}^{2}$ (abaixo) pela equação (B.3), mostrando a convergência com o aumento de $j$, comparada com o resultado da equação (A.9), em função de $x$ (esquerda) e $W$ (direita) . . . . . . . . . . . . . . . . . 90

B.2 Razão entre a TMC $F_{1}$, equação (B.3), em $Q^{2}=1 \mathrm{GeV}^{2}$ (acima), $Q^{2}=2 \operatorname{GeV}^{2}$ (meio) e $Q^{2}=5 G_{e V}^{2}$ (abaixo) pela TMC dada pela equação (A.9) em função de $x$ (esquerda) e $W$ (direita), mostrando a convergência com o aumento de $j$. . . . . . . . . . . . . . . . . 91

B.3 TMC para $F_{3}$ em $Q^{2}=1 G e V^{2}$ (acima), $Q^{2}=2 G e V^{2}$ (meio) e $Q^{2}=$ $5 \mathrm{GeV}^{2}$ (abaixo) pela equação (B.8), mostrando a convergência com o aumento de $j$, comparada com o resultado da equação (A.21), em função de $x$ (esquerda) e $W$ (direita) . . . . . . . . . . . . . . . 95

B.4 Razão entre a TMC $F_{3}$, equação (B.8), em $Q^{2}=1 \mathrm{GeV}^{2}$ (acima), $Q^{2}=2 \operatorname{GeV}^{2}$ (meio) e $Q^{2}=5 G_{e V}^{2}$ (abaixo) pela TMC dada pela equação (A.21) em função de $x$ (esquerda) e $W$ (direita), mostrando a convergência com o aumento de $j$. . . . . . . . . . . . . . . . . . 96 


\section{Introdução}

O estudo da estrutura interna do nucleon é realizado por intermédio de espalhamentos, utilizando para isto os aceleradores de partículas. Estas experiências podem ser feitas, por exemplo, com a colisão de léptons com o nucleon $\left(l+n \rightarrow l^{\prime}+X\right.$, onde $X$ pode representar o próprio nucleon, no espalhamento elástico, ou qualquer estado final permitido pelas leis de conservação, no espalhamento inelástico).

Bjorken em 1969, verificou que se o nucleon fosse composto de férmions puntiformes eles carregariam certa fração $x$ de momento do nucleon no referencial de Breit [1], e no chamado limite de Bjorken $Q^{2} \rightarrow \infty$ e $\nu \rightarrow \infty$ (virtualidade do fóton incidente e a energia transferida para o nucleon, respectivamente), a função de estrutura passava a ser somente dependente da variável $x$, que ficou conhecido como scaling de Bjorken. Estas predições foram confirmadas pelo Stanford Linear Accelerator Center (SLAC) [2] no mesmo ano. Ainda em 1969, Feynman motivado por este experimento, propôs o modelo de pártons [3, 4], que considera o nucleon como sendo composto por partículas puntiformes, o qual ele denominou de pártons, confinadas, não interagentes e carregadas eletricamente, que foram mais tarde relacionadas com os quarks não interagentes ${ }^{1}$ de Gell-Mann [5] e Zweig [6].

Com os dados do experimento realizado pela colaboração SLAC-MIT em 1972 [7], verificou-se que os pártons carregam aproximadamente metade do momento total do nucleon e a outra metade deve ser carregada por partículas que seriam eletricamente

\footnotetext{
${ }^{1}$ Feynman argumentou que no espalhamento lépton-nucleon os pártons não deveriam interagir, já que no limite do nucleon com momento infinito, as interações entre pártons são muito mais lentas do que a interação com o lépton incidente [4].
} 
neutras, que posteriormente receberam o nome glúons, os mediadores da interação forte que é descrita pela Cromodinâmica Quântica (Quantum Chromodynamics - QCD) [8]. Descoberto experimentalmente em 1978 no estudo do decaimento da ressonância $\Upsilon(9460)$ que é mediado por 3 glúons [9]. O scaling de Bjorken no âmbito da QCD deixa de ser verdadeiro, principalmente na região de pequeno $x$, devido à radiação dos glúons com bastante momento, que levam a violações logarítmicas do scaling [10]. Essa radiação produz uma evolução nas funções de estrutura, conforme $Q^{2}$ aumenta, mais e mais glúons são irradiados [10]. A confirmação experimental foi obtida com base nos dados do HERA [11, 12].

Sendo o nucleon composto por diversos pártons, o seu estudo é feito utilizando a função de distribuição de pártons (PDF) que representa a densidade de probabilidade de encontrar um párton carregando uma fração $x$ do momento do nucleon em uma dada energia $Q^{2}$. A sua evolução com respeito a $Q^{2}$ é descrita pelas equações DGLAP [13]. Atualmente existem diversos grupos que estudam esta área, por exemplo, CTEQ [14], GRV/GJR [15], MSTW [16], ABKM [17] e CJ12 [18] e suas diferenças são devido à escolha dos dados utilizados, uma escala de energia em $Q^{2}$ inicial, a parametrização das distribuições de pártons, tratamento dos erros dos dados, suposições teóricas, entre outros [19].

A altas energias a evolução em $Q^{2}$ leva, para pequeno $x$, a um crescimento exagerado do número de glúons. A solução deste problema é a recombinação destes glúons, ou seja, existe um limite de saturação do número destes pártons [20]. Embora este problema esteja na região de pequeno $x$, incertezas na região de grande $x$ são levadas para pequeno $x$ quando se evolui as equações DGLAP $[21,22]$.

Os experimentos recentes envolvendo lépton e nucleons se tornaram bastante precisos. Assim, é importante considerar todas as fontes de correções que podem contribuir na análise dos dados, como as correções radiativas eletrofracas [23], as massas dos quarks [24] e as correções da massa do alvo (TMC) [25, 26]. Neste trabalho iremos focar nas TMC, que são correções, do tipo $1 / Q^{2}$, de origem puramente cinemática nas funções de estrutura. 
O entendimento das TMC é importante por diversas razões. Seus efeitos são notáveis em grande $x$ de Bjorken e $Q^{2}$ pequeno, região que as PDFs não são muito bem definidas. Logo, um estudo confiável das PDFs deve incluir uma descrição precisa das TMC, além da interpretação dos dados com correlações de multiparton com pequena transferência de momento [27].

As TMC nas funções de estrutura foram determinadas primeiro por Georgi e Politzer em 1976 [26] com o uso da expansão do produto de operadores (OPE) [28] em ordem dominante da QCD. Nesta abordagem o resultado final passa a depender de uma nova variável de scaling, a variável de Nachtmann $\xi$ [25] (que é a fração do momento do nucleon no cone de luz que o párton carrega), ao invés de $x$. Isto leva ao chamado threshold problem, que estende as funções de estrutura para regiões fisicamente proibidas e que foram percebidos nos primórdios da QCD [29, 30].

No primeiro capítulo, apresenta-se a evolução da estrutura interna do nucleon que é provada quando ocorre uma transferência muito grande de momento do projétil para o alvo. Em uma primeira aproximação, no modelo de quarks, este espalhamento pode ser interpretado a partir do espalhamento entre um lépton e um único quark, $l+q \rightarrow l^{\prime}+q^{\prime}$, ou seja, um espalhamento eletromagnético entre duas partículas puntuais de spin $\frac{1}{2}$, também é mostrado como essas idéias trouxeram informações a respeito da composição do nucleon.

O segundo capítulo foi dedicado a mostrar a ferramenta matemática chamada Expansão do Produto de Operadores (Operator Product Expansion - OPE) proposta originalmente por Wilson em 1969 [28] que permite separar a seção de choque, e consequentemente as funções de estrutura, em uma região onde a liberdade assintótica é válida (QCD perturbativa, curtas distâncias) e onde não é (QCD não perturbativa, longas distâncias) e então obtemos os momentos das funções de estrutura para o próton.

Quando a virtualidade do fóton incidente $\left(Q^{2}\right)$ no espalhamento profundamente inelástico (Deep Inelastic Scattering - DIS) é pequena, da ordem da massa do alvo (como o próton, por exemplo), é preciso considerar efeitos puramente cinemáticos 
devido a massa do alvo ser comparável à escala de energia do fóton incidente, esta correção é chamada de correções da massa do alvo (Target Mass Corrections - TMC). Assim, o terceiro capítulo apresenta o surgimento desta correção e possíveis soluções, além de mostrar as falhas nas tentativas de solucionar este problema.

No quarto capítulo propomos uma solução para o problema que se encontra ao incluir as TMC nos cálculos das funções de estrutura, que consiste em não utilizar a variável de scaling $\xi$ que aparece naturalmente quando se considera as TMC, então obtemos para as funções de estrutura uma série de potências e realizamos um estudo numérico por intermédio de vários gráficos que são apresentados no final deste capítulo.

Por fim a conclusão está no quinto capítulo. Segue-se também dois apêndices em que é feito detalhadamente a dedução das funções de estrutura $\left(F_{1}, F_{L}, F_{3}, F_{4}, F_{5}\right)$ com as TMC, sendo o primeiro com a variável de scaling $\xi$ e o segundo sem esta variável (chegando na série de potências). 


\section{Capítulo 1}

\section{Estrutura Interna do Nucleon}

Este capítulo trata de como foi descoberta a estrutura interna do nucleon, utilizando para isto espalhamentos (elásticos e inelásticos). Para tanto se fez uma revisão do espalhamento elétron-múon. Em seguida estudou-se o espalhamento elétron-próton elástico, onde foi observada a estrutura extensa do próton, e o espalhamento elétronpróton inelástico que foi, em uma primeira análise, interpretado como uma soma de espalhamentos elásticos entre o elétron e uma partícula puntual. Estas partículas puntuais carregam uma certa fração do momento do nucleon e possuem spin $\frac{1}{2}$ (modelo de pártons) que foram, mais tarde, relacionadas com os quarks e glúons da QCD e comentamos sobre a existência da assimetria no mar de quarks e o problema do spin do nucleon.

\subsection{Espalhamento elétron-múon}

O espalhamento eletromagnético entre duas partículas puntuais de spin $\frac{1}{2}$ mais simples é:

$$
e+\mu \rightarrow e^{\prime}+\mu^{\prime}
$$

Em ordem mais baixa na teoria de perturbação da Eletrodinâmica Quântica ( Quantum Electrodynamics - QED) o espalhamento é descrito pela troca de um fóton, esquema- 
tizado na Figura (1.1).

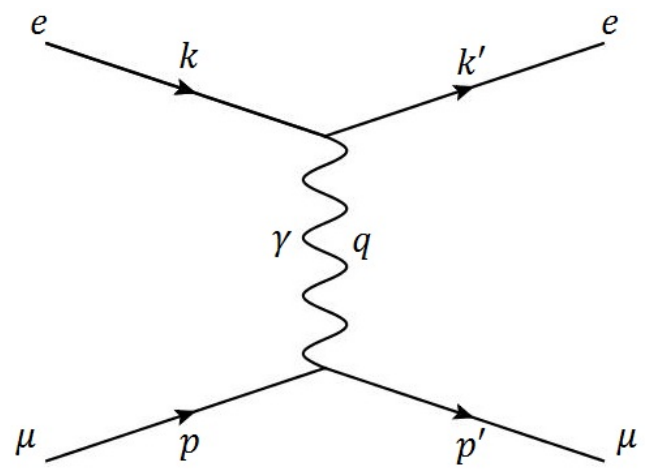

Figura 1.1: Diagrama de Feynman em ordem mais baixa para o espalhamento $e+\mu \rightarrow e^{\prime}+\mu^{\prime}$.

Utilizando as regras de Feynman, a amplitude invariante do espalhamento é:

$$
\mathcal{M}=-\frac{4 \pi \alpha}{q^{2}}\left[\bar{u}_{e}\left(k^{\prime}\right) \gamma_{\alpha} u_{e}(k)\right]\left[\bar{u}_{\mu}\left(p^{\prime}\right) \gamma^{\alpha} u_{\mu}(p)\right]
$$

onde $q=k-k^{\prime}$ e $\alpha$ a constante de estrutura fina.

A seção de choque diferencial para um elétron espalhado dentro de um ângulo sólido $d \Omega$ e com energia final $E^{\prime} \rightarrow E^{\prime}+d E^{\prime}$ no sistema de laboratório $\left(p=\left(m_{\mu}, 0\right), k=\right.$ $\left.(E, \mathbf{k}), k^{\prime}=\left(E^{\prime}, \mathbf{k}^{\prime}\right)\right)$ pode ser escrita como [31]:

$$
\frac{d^{2} \sigma}{d \Omega d E^{\prime}}=\frac{E^{\prime}}{2 m_{\mu} E 16 \pi^{2}}|\overline{\mathcal{M}}|^{2} \frac{\delta\left(p_{0}^{\prime}-p_{0}-q_{0}\right)}{2 p_{0}^{\prime}},
$$

onde o quadrado da média da amplitude é:

$$
\begin{aligned}
|\overline{\mathcal{M}}|^{2}= & \frac{16 \pi^{2} \alpha^{2}}{q^{4}}\left(\frac{1}{2} \sum_{\text {spins }}\left[\bar{u}_{e}\left(k^{\prime}\right) \gamma_{\alpha} u_{e}(k)\right]^{*}\left[\bar{u}_{e}\left(k^{\prime}\right) \gamma_{\beta} u_{e}(k)\right]\right) \times \\
& \times\left(\frac{1}{2} \sum_{\text {spins }}\left[\bar{u}_{\mu}\left(p^{\prime}\right) \gamma^{\alpha} u_{\mu}(p)\right]^{*}\left[\bar{u}_{\mu}\left(p^{\prime}\right) \gamma^{\beta} u_{\mu}(p)\right]\right) \\
= & \frac{16 \pi^{2} \alpha^{2}}{q^{4}} L_{\alpha \beta}(e, e) W^{\alpha \beta}(\mu, \mu),
\end{aligned}
$$

sendo $L_{\alpha \beta}(e, e)$ e $W^{\alpha \beta}(\mu, \mu)$ os tensores leptônicos do elétron e múon, respectivamente. 
Para o elétron:

$$
\begin{aligned}
L_{\alpha \beta}(e, e) & =\frac{1}{2} \sum_{\text {spins }}\left[\bar{u}_{e}\left(k^{\prime}\right) \gamma_{\alpha} u_{e}(k)\right]^{*}\left[\bar{u}_{e}\left(k^{\prime}\right) \gamma_{\beta} u_{e}(k)\right] \\
& =\frac{1}{2} \operatorname{Tr}\left[\left(\not k^{\prime}+m_{e}\right) \gamma_{\alpha}\left(\not k+m_{e}\right) \gamma_{\beta}\right] \\
& =2\left[k_{\alpha} k_{\beta}^{\prime}+k_{\alpha}^{\prime} k_{\beta}-g_{\alpha \beta}\left(k \cdot k^{\prime}-m_{e}^{2}\right)\right] .
\end{aligned}
$$

Analogamente para o múon:

$$
W^{\alpha \beta}(\mu, \mu)=2\left[p^{\alpha} p^{\beta^{\prime}}+p^{\alpha^{\prime}} p^{\beta}-g^{\alpha \beta}\left(p \cdot p^{\prime}-m_{\mu}^{2}\right)\right]
$$

No regime de altas energias, pode-se desprezar a massa do elétron incidente e temos para a transferência de momento:

$$
Q^{2}=-q^{2}=-\left(k-k^{\prime}\right)^{2} \simeq 2 k \cdot k^{\prime}=4 E^{\prime} \operatorname{sen}^{2}\left(\frac{\theta}{2}\right)
$$

onde $\theta$ é o ângulo de espalhamento do elétron incidente. Introduz-se uma nova variável, $Q^{2}=-q^{2}$, chamada de virtualidade do fóton, tal que $Q^{2} \geq 0$.

Substituindo as equações (1.4)-(1.7) em (1.3), e com um pouco de álgebra, obtemos a seção de choque no sistema do laboratório:

$$
\frac{d^{2} \sigma}{d \Omega d E^{\prime}}=\frac{\alpha^{2}}{4 E^{2} \operatorname{sen}^{4}(\theta / 2)}\left[\cos ^{2}\left(\frac{\theta}{2}\right)+\frac{Q^{2}}{2 m_{\mu}^{2}} \operatorname{sen}^{2}\left(\frac{\theta}{2}\right)\right] \delta\left(\nu-\frac{Q^{2}}{2 m_{\mu}}\right)
$$

$\mathrm{Ou}$

$$
\frac{d \sigma}{d \Omega}=\left(\frac{d \sigma}{d \Omega}\right)_{M o t t}\left[1+\frac{Q^{2}}{2 m_{\mu}^{2}} \tan ^{2}\left(\frac{\theta}{2}\right)\right]\left[1+\frac{2 E}{m_{\mu}} \operatorname{sen}^{2}\left(\frac{\theta}{2}\right)\right]^{-1}
$$


onde a seção de choque de Mott [32], em altas energias, é

$$
\left(\frac{d \sigma}{d \Omega}\right)_{M o t t}=\frac{\alpha^{2} \cos ^{2}\left(\frac{\theta}{2}\right)}{4 E^{2} \operatorname{sen}^{4}\left(\frac{\theta}{2}\right)}
$$

A seção de choque dada pela equação (1.10) é o espalhamento de uma partícula de

spin $\frac{1}{2}$ pelo campo coulombiano de um alvo massivo e sem spin. Os fatores extras em (1.9) são devidos ao fato que o alvo possui spin $\frac{1}{2}$, assim há uma interação magnética entre as partículas, e ao fato que o alvo possui massa finita recuando durante o espalhamento.

\subsection{Espalhamento elétron-próton}

\subsubsection{Espalhamento elástico}

Se o próton fosse uma partícula puntual, a seção de choque poderia ser obtida fazendo a mudança $m_{\mu} \rightarrow m_{p}$ na equação (1.9). Porém, espalhamentos elásticos com feixes de elétrons mostraram que o nucleon é extenso [33], possuindo os chamados fatores de forma.

Utilizando as regras de Feynman para o processo esquematizado na Figura (1.2), obtêm-se a amplitude invariante:

$$
\mathcal{M}=-\frac{4 \pi \alpha}{q^{2}}\left[\bar{u}_{e}\left(k^{\prime}\right) \gamma_{\alpha} u_{e}(k)\right]\left[\bar{u}_{p}\left(p^{\prime}\right) \Gamma^{\alpha} u_{p}(p)\right]
$$

Notemos que $\gamma^{\alpha}$ foi substituído por $\Gamma^{\alpha}$ porque não temos uma teoria quântica de campos para um objeto extenso como o próton, logo não sabemos qual a expressão para o vértice $\gamma-p$.

No entanto, $\Gamma^{\alpha}$ pode ser parametrizado usando uma combinação entre as matrizes de Dirac, permitida pela conservação de paridade e invariância sobre reversão tempo- 


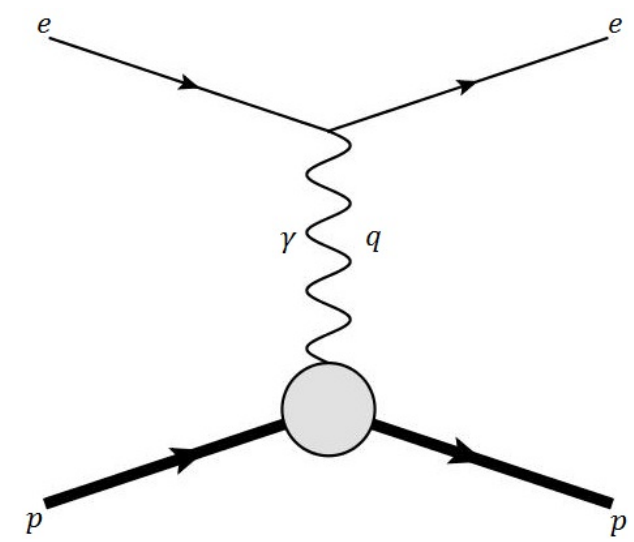

Figura 1.2: Diagrama de Feynman em ordem mais baixa para o espalhamento $e+p \rightarrow e^{\prime}+p^{\prime}$, onde a bola no vértice $\gamma-p$ esquematiza os fatores de forma.

ral, simetrias que devem ser respeitadas na interação eletromagnética:

$$
\Gamma^{\alpha}=F_{1}\left(q^{2}\right) \gamma^{\alpha}+F_{2}\left(q^{2}\right) \frac{\kappa}{2 m_{p}} i \sigma^{\alpha \beta} q_{\beta}+F_{3}\left(q^{2}\right) q^{\alpha}
$$

onde $2 \sigma^{\alpha \beta}=i\left[\gamma^{\alpha}, \gamma^{\beta}\right]$ e $\kappa$ é o momento magnético anômalo do próton ${ }^{1}(\kappa \simeq 1.79$ em magnetons de Bohr).

Os $F_{i}\left(q^{2}\right)$ são os chamados fatores de forma eletromagnéticos elásticos ${ }^{2}$ que só dependem do momento transferido $q$ e que descrevem quão diferente o próton é de uma partícula puntual. No caso do espalhamento ser puramente eletromagnético a corrente é conservada, assim $F_{3}\left(q^{2}\right) \equiv 0$. Os fatores de forma parametrizam nossa ignorância da estrutura detalhada do próton.

No caso em que o fóton tenha pouco momento (grande comprimento de onda) ${ }^{3}$ não importa se o próton possui estrutura menor que 1 fermi [31]. Neste limite são

\footnotetext{
${ }^{1}$ É chamado de anômalo pois medidas do momento magnético do próton realizadas por Stern em 1933 [34] revelaram que seu valor era aproximadamente 2.8 vezes maior do que o esperado para uma partícula de spin $\frac{1}{2}$ e puntual.

${ }^{2} F_{1}\left(q^{2}\right)$ é usualmente chamado de fator de forma de Dirac, que está associado com o desvio de uma partícula puntual carregada com spin $\frac{1}{2}$ e $F_{2}\left(q^{2}\right)$ de fator de forma de Pauli, que seria o desvio de um momento magnético anômalo puntual.

${ }^{3}$ Este limite corresponde com o próton interagindo com um campo eletromagnético estático.
} 
escolhidos (para o próton) $)^{4}$

$$
F_{1}(0)=F_{2}(0)=1 .
$$

O procedimento para o cálculo da seção de choque para o processo $e+p \rightarrow e^{\prime}+p^{\prime}$ é o mesmo feito na Seção 1.1 com a mudança de $\gamma^{\alpha}$ no vértice do múon para $\Gamma^{\alpha}$ no vértice do próton:

$$
\frac{d^{2} \sigma}{d \Omega d E^{\prime}}=\frac{\alpha^{2} E^{\prime}}{2 m_{p} q^{4} E} L_{\alpha \beta}(e, e) W^{\alpha \beta}(p, p) \frac{\delta\left(p_{0}^{\prime}-p_{0}-q_{0}\right)}{2 p_{0}^{\prime}}
$$

onde $W^{\alpha \beta}(p, p)=\frac{1}{2} \operatorname{Tr}\left[\left(\not p^{\prime}+m_{p}\right) \Gamma^{\alpha}\left(\not p+m_{p}\right) \Gamma^{\beta}\right]$.

Substituindo as equações (1.5) e (1.7) em (1.14), chega-se (no sistema de laboratório) à fórmula de Rosenbluth [35]:

$$
\begin{aligned}
\frac{d^{2} \sigma}{d \Omega d E^{\prime}} & =\frac{\alpha^{2}}{4 E^{2} \operatorname{sen}^{4}(\theta / 2)}\left[\left(F_{1}^{2}\left(q^{2}\right)+\frac{\kappa Q^{2}}{4 m_{p}^{2}} F_{2}^{2}\left(q^{2}\right)\right) \cos ^{2}\left(\frac{\theta}{2}\right)+\right. \\
& \left.+\frac{Q^{2}}{2 m_{p}^{2}}\left(F_{1}^{2}\left(q^{2}\right)+\kappa F_{2}^{2}\left(q^{2}\right)\right)^{2} \operatorname{sen}^{2}\left(\frac{\theta}{2}\right)\right] \delta\left(\nu-\frac{Q^{2}}{2 m_{p}}\right)
\end{aligned}
$$

ou:

$$
\begin{aligned}
\frac{d \sigma}{d \Omega} & =\left(\frac{d \sigma}{d \Omega}\right)_{\text {Mott }}\left[\left(F_{1}^{2}\left(q^{2}\right)+\frac{\kappa Q^{2}}{4 m_{p}^{2}} F_{2}^{2}\left(q^{2}\right)\right)+\right. \\
& \left.+\frac{Q^{2}}{2 m_{p}^{2}}\left(F_{1}^{2}\left(q^{2}\right)+\kappa F_{2}^{2}\left(q^{2}\right)\right)^{2} \tan ^{2}\left(\frac{\theta}{2}\right)\right]\left[1+\frac{2 E}{m_{p}} \operatorname{sen}^{2}\left(\frac{\theta}{2}\right)\right]^{-1}
\end{aligned}
$$

Fazendo $F_{1}=1, F_{2}=0$ e $m_{p}=m_{\mu}$, em (1.15) ou (1.16), obtemos a seção de choque para $e+\mu \rightarrow e^{\prime}+\mu^{\prime}$, assim pode-se dizer que o fator de forma elástico para uma partícula puntual, como o $\mu$, é uma constante independente de $Q^{2}$.

Usualmente, utiliza-se uma combinação linear dos fatores de forma, os chamados

\footnotetext{
${ }^{4}$ Se o alvo fosse um nêutron, escolhe-se $F_{1}(0)=0, F_{2}(0)=1$ e experimentalmente $\kappa \simeq-1.91$.
} 
fatores de forma elétricos $\left(G_{E}\left(q^{2}\right)\right)$ e magnéticos $\left(G_{M}\left(q^{2}\right)\right)$ de Sachs [36]:

$$
G_{E}\left(q^{2}\right)=F_{1}\left(q^{2}\right)+\frac{\kappa q^{2}}{4 m_{p}^{2}} F_{2}\left(q^{2}\right) \quad \text { e } \quad G_{M}\left(q^{2}\right)=F_{1}\left(q^{2}\right)+\kappa F_{2}\left(q^{2}\right)
$$

com eles não se tem termos de interferência, do tipo $F_{1}^{2} F_{2}^{2}$, que aparecem em (1.15) e (1.16).

Através da Figura (1.3), vê-se que:

$$
G_{E}\left(q^{2}\right) \propto G_{M}\left(q^{2}\right) \propto \frac{1}{\left(1+Q^{2} / 0.71\right)^{2}}
$$

a chamada forma de dipolo [31].

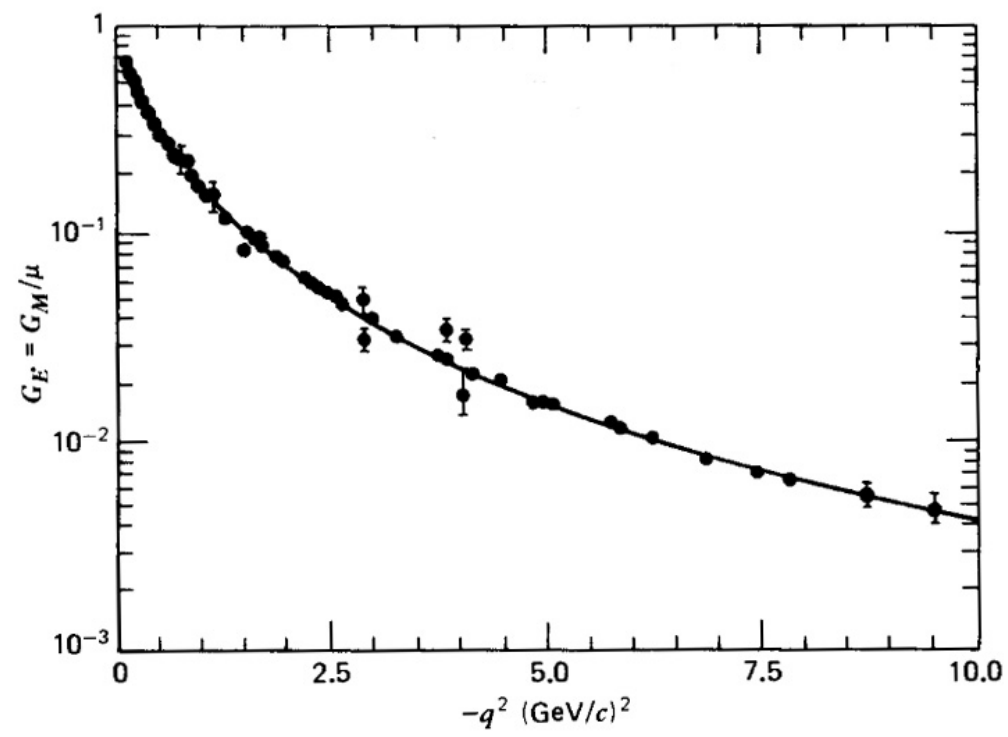

Figura 1.3: Fatores de forma de Sachs para o próton em função de $q^{2}$. Extraído de [31].

Como a seção de choque de Mott, equação (1.10), decresce como $1 / Q^{4}$, a seção de choque elástica se anula rapidamente para alto $Q^{2}$, dificultando a obtenção de medidas precisas. Não obstante, medidas cada vez mais precisas em alto $Q^{2}$ vem sendo feitas no JLab, podendo assim refinar os modelos existentes para os fatores de forma eletromagnéticos [37]. 


\subsubsection{Espalhamento inelástico}

Em energias moderadas, o espalhamento elétron-próton é necessariamente elástico (ele recua, mas continua sendo um próton), mas se o elétron incidente carregar energia o suficiente (grande $Q^{2}$ ) se melhora a resolução com que observamos o próton. Caso $Q^{2}$ seja moderado o próton pode entrar no estado da ressonância $\Delta(1232)$ e então produzir mésons $\pi\left(e+p \rightarrow e^{\prime}+\Delta^{+} \rightarrow e^{\prime}+p^{\prime}+\pi^{0}\right.$, veja Figura (1.4)). Se $Q^{2}$ for muito grande, o próton acaba perdendo sua identidade e se quebrando em diversas outras partículas $\left(e+p \rightarrow e^{\prime}+X\right.$, onde $X$ representa a soma de todos estados hadrônicos criados após a colisão, Figura (1.5)), quando somente se mede o ângulo e a energia do lépton espalhado este processo recebe o nome de Espalhamento Profundamente Inelástico (Deep Inelastic Scattering - DIS) ${ }^{5}$. Panofsky em 1968 [39] descobriu que os análogos aos fatores de forma no DIS não diminuem com o aumento de $Q^{2}$, o que foi a base para a idéia de que o nucleon possui uma subestrutura interna.

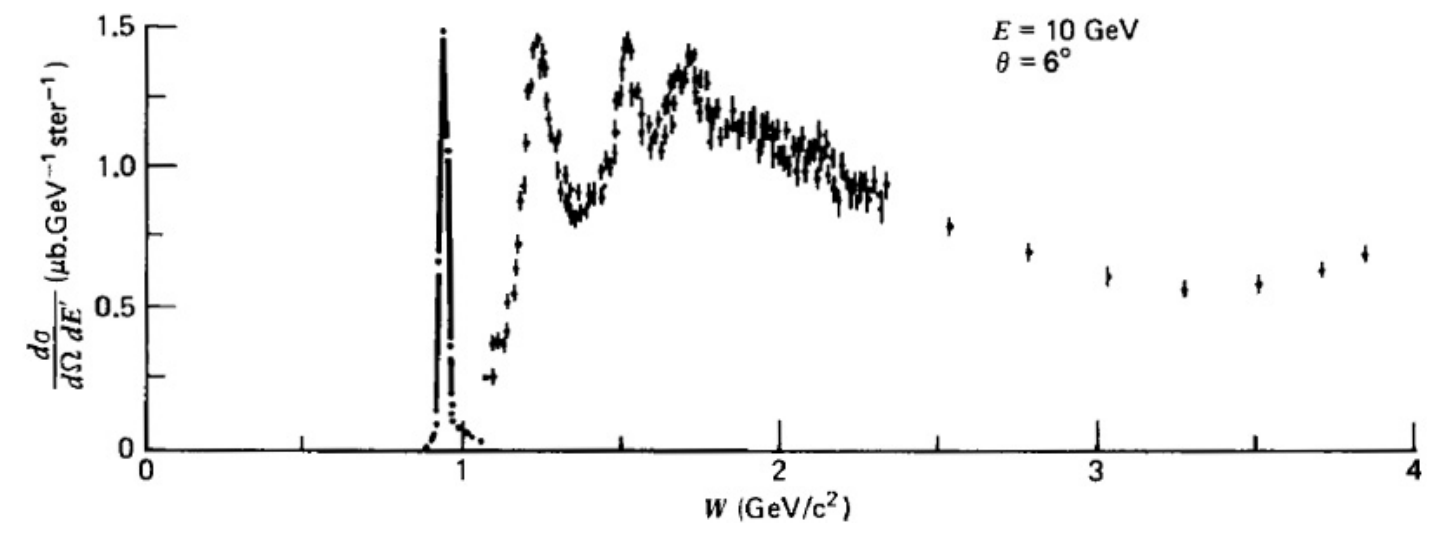

Figura 1.4: Seção de choque para $e+p \rightarrow e^{\prime}+X$ em função da massa do sistema hadrônico $X$ produzido, $W^{2}=(p+q)^{2}$. O pico elástico em $W=M$ foi reduzido por um fator de 8.5. Extraído de [31].

Novamente, a seção de choque é descrita como:

$$
\frac{d^{2} \sigma}{d \Omega d E^{\prime}}=\frac{\alpha^{2} E^{\prime}}{2 m_{p} q^{4} E} L_{\alpha \beta}(e, e) W^{\alpha \beta}(p, X),
$$

\footnotetext{
${ }^{5} \mathrm{O}$ DIS também é chamado de espalhamento inclusivo, que são eventos onde não se identifica experimentalmente o estado final do alvo, somente o do projétil [38].
} 
o tensor leptônico $\left(L_{\alpha \beta}(e, e)\right)$ continua inalterado, porém $W^{\alpha \beta}(p, X)$ corresponde a transições eletromagnéticas do próton para todos os possíveis estados finais:

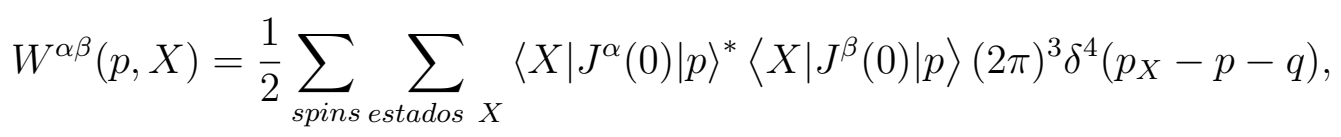

onde $p_{X}$ é o quadri-momento total do estado $|X\rangle$.

Como interações eletromagnéticas conservam paridade, todos os momentos são integrados exceto $p, q$ e $p_{X}=p+q$ (pela função $\delta$ ) e para o espalhamento não polarizado se soma todos os spins. Além disso, $W^{\alpha \beta}(p, X)$ tem que ser um tensor de rank-2 e depender somente de $p, q$ e $g^{\alpha \beta}$, assim:

$$
\begin{array}{r}
W^{\alpha \beta}(p, X)=A\left(q^{2}, \nu\right) g^{\alpha \beta}+B\left(q^{2}, \nu\right) q^{\alpha} q^{\beta}+C\left(q^{2}, \nu\right)\left(q^{\alpha} p^{\beta}+q^{\beta} p^{\alpha}\right)+ \\
+D\left(q^{2}, \nu\right) p^{\alpha} p^{\beta}
\end{array}
$$

onde no sistema de laboratório, $\nu=E-E^{\prime}=\frac{q \cdot p}{m_{p}}$.

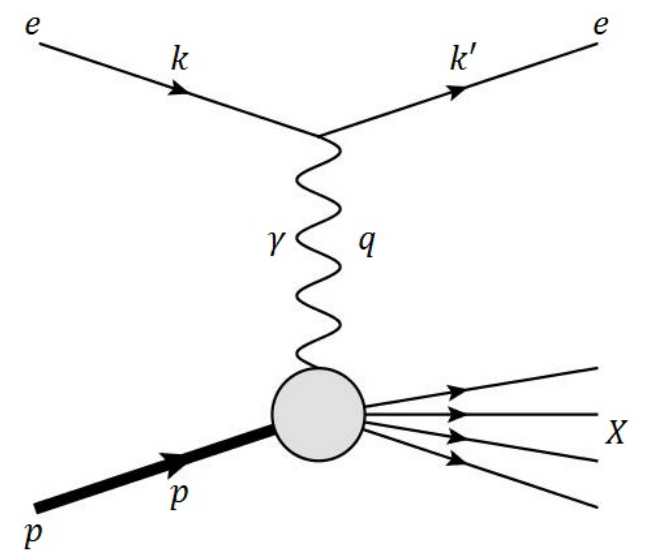

Figura 1.5: Diagrama de Feynman em ordem dominante do espalhamento $e+p \rightarrow e^{\prime}+X$

A conservação da corrente eletromagnética implica em $q_{\alpha} W^{\alpha \beta}=W^{\alpha \beta} q_{\beta}=0$, que leva em $B=-\frac{A}{q^{2}}+D\left(\frac{p \cdot q}{q^{2}}\right)^{2}$ e $C=-D \frac{p \cdot q}{q^{2}}$ e escrevendo $W_{1}=-\frac{A}{2 m_{p}}$ e $W_{2}=-\frac{D m_{p}}{2}$, 
chega-se a:

$$
\begin{aligned}
\frac{W^{\alpha \beta}(p, X)}{2 m_{p}} & =\left[\frac{q^{\alpha} q^{\beta}}{q^{2}}-g^{\alpha \beta}\right] W_{1}\left(q^{2}, \nu\right)+ \\
& +\left[\left(p^{\alpha}-\frac{p \cdot q}{q^{2}} q^{\alpha}\right)\left(p^{\beta}-\frac{p \cdot q}{q^{2}} q^{\beta}\right)\right] \frac{W_{2}\left(q^{2}, \nu\right)}{m_{p}^{2}}
\end{aligned}
$$

As funções $W_{1}\left(q^{2}, \nu\right)$ e $W_{2}\left(q^{2}, \nu\right)$ são conhecidas como funções de estrutura do nucleon, sendo a generalização para o caso inelástico dos fatores de forma elásticos.

Substituindo as equações (1.5) e (1.22) em (1.19), no sistema de laboratório $(p \cdot k=$ $\left.m_{p}, p \cdot k^{\prime}=m_{p} E^{\prime}\right)$ e com a equação $(1.7)$, tem-se:

$$
\frac{d^{2} \sigma}{d \Omega d E^{\prime}}=\frac{\alpha^{2}}{4 E^{2} \operatorname{sen}^{4}(\theta / 2)}\left[2 W_{1}\left(q^{2}, \nu\right) \operatorname{sen}^{2}\left(\frac{\theta}{2}\right)+W_{2}\left(q^{2}, \nu\right) \cos ^{2}\left(\frac{\theta}{2}\right)\right] .
$$

Comparando a seção de choque (1.23) com (1.15), pode-se determinar o limite elástico das funções de estrutura:

$$
\begin{aligned}
W_{1}^{e+p \text { elástica }} & =\frac{Q^{2}}{4 m_{p}^{2}}\left(F_{1}^{2}+\kappa F_{2}^{2}\right)^{2} \delta\left(\nu-\frac{Q^{2}}{2 m_{p}}\right) \\
W_{2}^{e+p \text { elástica }} & =\left(F_{1}^{2}+\frac{\kappa^{2} Q^{2}}{4 m_{p}^{2}} F_{2}^{2}\right) \delta\left(\nu-\frac{Q^{2}}{2 m_{p}}\right) .
\end{aligned}
$$

Se o espalhamento fosse ${ }^{6} e+\mu \rightarrow e^{\prime}+X$ :

$$
\begin{aligned}
2 m_{\mu} W_{1}^{e+\mu ~ e l a ́ s t i c a ~} & =\frac{Q^{2}}{2 m_{\mu} \nu} \delta\left(\frac{Q^{2}}{2 m_{\mu} \nu}-1\right) ; \\
\nu W_{2}^{e+\mu ~ e l a ́ s t i c a ~} & =\delta\left(\frac{Q^{2}}{2 m_{\mu} \nu}-1\right),
\end{aligned}
$$

que depende somente da razão adimensional $\omega=\frac{2 m \nu}{Q^{2}}$, assim não é preciso nenhuma escala de massa como na equação (1.24).

Pode-se reinterpretar as funções de estrutura da forma apresentada na Figura (1.6),

\footnotetext{
${ }^{6}$ Caso de uma partícula com spin $\frac{1}{2}$ e puntiforme.
} 


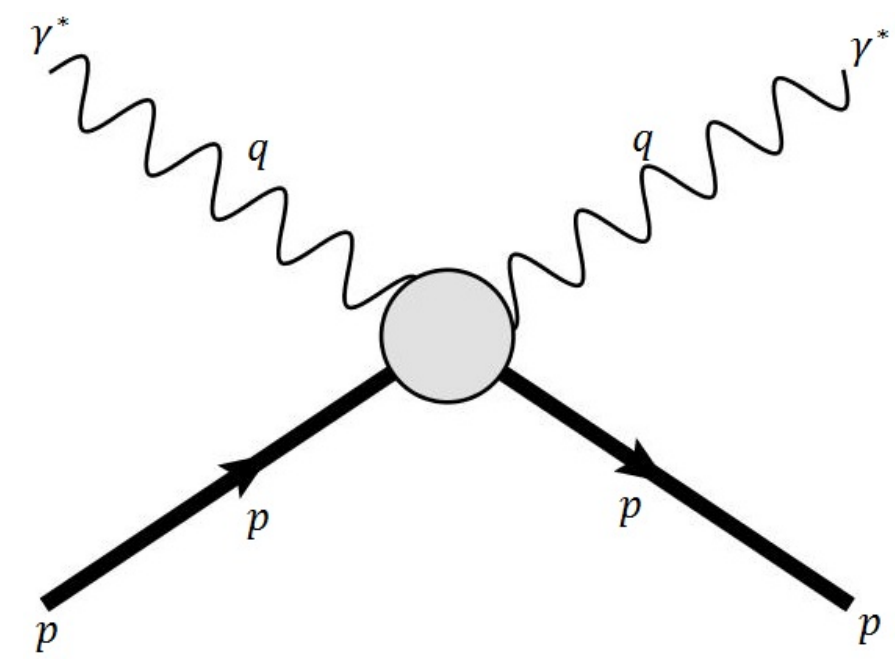

Figura 1.6: Diagrama de Feynman para o espalhamento Compton virtual.

onde $\gamma^{*}$ indica que o fóton está fora de sua camada de massa (fóton virtual de massa $q^{2}$ ). Assim o tensor hadrônico, equação (1.20), pode ser relacionado, via teorema óptico, com a parte imaginária (absortiva) da amplitude de Feynman para o espalhamento do fóton virtual com o próton (amplitude do espalhamento Compton virtual) [36], algo que será explorado com alguma profundidade no próximo capítulo.

\subsection{Modelo de pártons e o scaling de Bjorken}

Bjorken [40] argumentou que em altas energias as funções de estrutura inelásticas se tornam independentes de $q^{2}$, ficando somente como função da razão:

$$
x=\frac{1}{\omega}=\frac{Q^{2}}{2 p \cdot q} .
$$

No sistema de laboratório assume a forma:

$$
x=\frac{1}{\omega}=\frac{Q^{2}}{2 m_{p} \nu},
$$

esta variável é chamada de $x$ de Bjorken e pode ser interpretada como a fração do momento do nucleon que o párton carrega. Ela pode assumir valores entre $0 \leq x \leq 1$, 
como pode ser verificado abaixo:

$$
x=\frac{Q^{2}}{2 p \cdot q}=\frac{Q^{2}}{(p+q)^{2}-p^{2}+Q^{2}}=\frac{Q^{2}}{W^{2}-m_{p}^{2}+Q^{2}}=\frac{1}{1+\left(W^{2}-m_{p}^{2}\right) / Q^{2}}
$$

Como o limite inferior para a massa final é $W=m_{p}$ (correspondendo ao espalhamento elástico) se tem $x=1$ para um limite superior e como $Q^{2} \geq 0$, tem-se $x=0$ para um limite inferior.

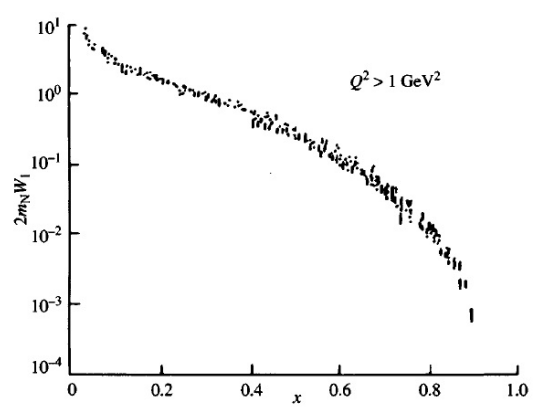

Figura 1.7: Comportamento de $2 m_{p} W_{1}$, não se nota variação

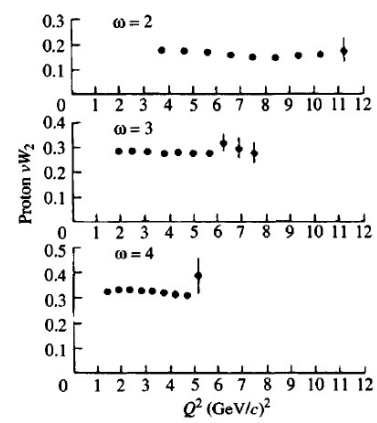

Figura 1.8: Comportamento de $\nu W_{2}$ para vários $\omega$, não se nota variação significativa com $Q^{2}$. Extraído de [36].

A independência das funções de estrutura em $Q^{2}$, como observado na experiência original, pode ser visualizada nas Figuras (1.7) e (1.8). Essa propriedade aparece quando se assume que o momento transversal dos pártons é pequeno, no sistema em que o próton possui momento infinito [10].

A propriedade observada (independência em $Q^{2}$ ) é exatamente o que se encontra no espalhamento elástico de um elétron com uma partícula puntual de spin $\frac{1}{2}$ (como mostrado em (1.25)). Esta propriedade, prevista por Bjorken [1], é conhecida como scaling de Bjorken.

No entanto, a independência das funções de estrutura de $Q^{2}$ foi uma grande coincidência, já que elas dependem de $Q^{2}$. Dependência que é entendida dentro da QCD perturbativa, onde a radiação dos hard glúons dos quarks violam essa suposição, levando a violações no scaling logarítmicas, que são particularmente grande em pequeno $x$ [10], como pode ser visualizado na Figura (1.9). 


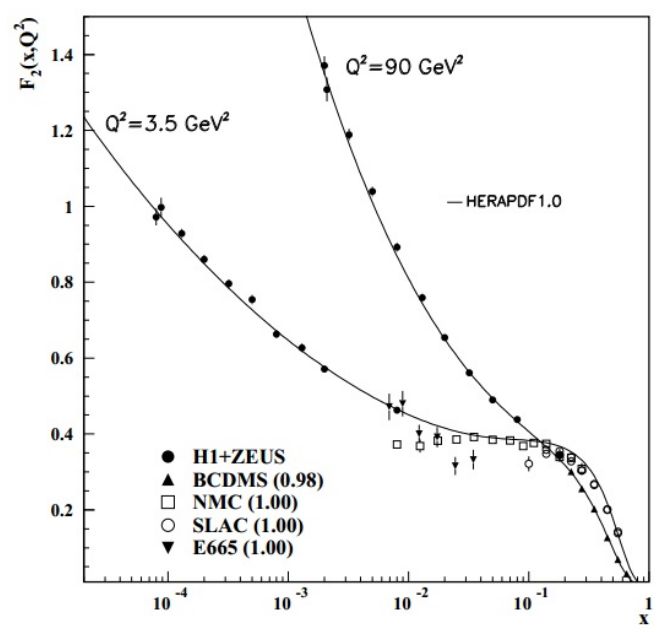

Figura 1.9: Evidência da quebra de scaling, os dados estão normalizados pelo valor em parêntesis. Extraído de [10].

Pode-se concluir que para uma partícula de massa $m_{i}=x m_{p}$ com carga $e_{i}$, a partir das equações (1.25) para um espalhamento elástico, que:

$$
\begin{aligned}
W_{1, i}^{\text {elástica }} & =e_{i}^{2} \frac{Q^{2}}{4 x^{2} m_{p}^{2} \nu} \delta\left(1-\frac{Q^{2}}{2 x m_{p} \nu}\right) ; \\
\nu W_{2, i}^{\text {elástica }} & =e_{i}^{2} \delta\left(1-\frac{Q^{2}}{2 x m_{p} \nu}\right) .
\end{aligned}
$$

Então, se o próton é composto de partículas puntuais de spin $\frac{1}{2}$ (os pártons de Feynman), o espalhamento inelástico esquematizado na Figura (1.5) pode ser substituído pela Figura (1.10), que representa o espalhamento de um elétron por um próton através da soma incoerente de espalhamentos elásticos do fóton virtual com os pártons.

No limite $Q^{2} \rightarrow \infty, \nu \rightarrow \infty$ com $x=\frac{Q^{2}}{2 m_{p} \nu}$ fixo ${ }^{7}$, é usual definir:

$$
\begin{array}{r}
m_{p} W_{1}\left(Q^{2}, \nu\right) \rightarrow F_{1}(x) ; \\
\nu W_{2}\left(Q^{2}, \nu\right) \rightarrow F_{2}(x) ;
\end{array}
$$

\footnotetext{
${ }^{7}$ Este limite é comumente chamado de limite de Bjorken.
} 


$$
F_{L}(x)=F_{2}(x)-2 x F_{1}(x)
$$

onde $F_{L}$ é a função de estrutura longitudinal.

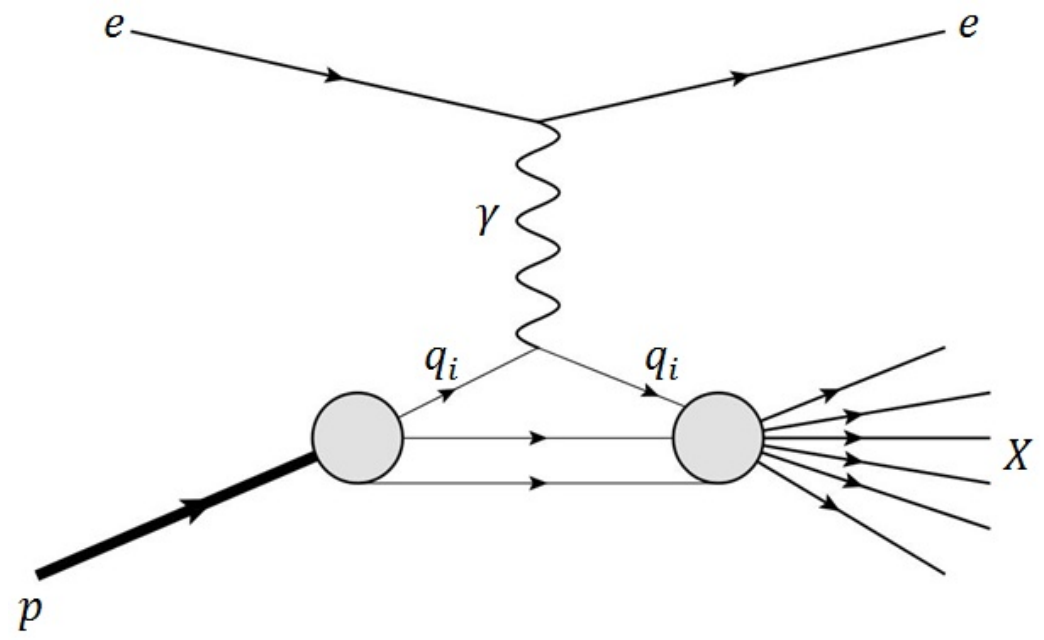

Figura 1.10: Interpretação do modelo de pártons para $e+p \rightarrow e^{\prime}+X, q_{i}$ representa o párton do tipo $i$.

A confirmação experimental do scaling de Bjorken [2] foi interpretada como a evidência de que o próton é composto por partículas puntiformes, e atualmente os pártons são identificados com os quarks e glúons ${ }^{8}$ da QCD.

Devido ao fato de que existe mais de um párton no interior do nucleon, é preciso somar sobre todos os $i$ pártons e integrar sobre todos os $x$, sendo a integral pesada pela densidade de probabilidade $f_{i}(x)$ de que o párton $i$ tenha fração $x$ do momento do nucleon:

$$
\begin{aligned}
W_{1}^{i}\left(Q^{2}, \nu\right) & =\sum_{i} \int_{0}^{1} d x f_{i}(x) e_{i}^{2} \frac{Q^{2}}{4 x^{2} m_{p}^{2} \nu} \delta\left(1-\frac{Q^{2}}{2 x m_{p} \nu}\right)=\sum_{i} \frac{e_{i}^{2} f_{i}(x)}{2 m_{p}} \\
\nu W_{2}^{i}\left(Q^{2}, \nu\right) & =\sum_{i} \int_{0}^{1} d x f_{i}(x) e_{i}^{2} \delta\left(1-\frac{Q^{2}}{2 x m_{p} \nu}\right)=x \sum_{i} e_{i}^{2} f_{i}(x)
\end{aligned}
$$

\footnotetext{
${ }^{8}$ Como os glúons possuem somente carga de cor, estes não participam no DIS $e+p$ em ordem dominante na QED. Sua contribuição passa a ser relevante quando se estuda o DIS utilizando a QCD perturbativa.
} 
onde $f_{i}(x)$ é comumente chamada de Função de Distribuição de Pártons (Parton Distribution Functions - PDF).

No limite de Bjorken (1.30):

$$
\begin{aligned}
m_{p} W_{1}^{i}\left(Q^{2}, \nu\right) & =\frac{1}{2} \sum_{i} e_{i}^{2} f_{i}(x)=F_{1}(x) \\
\nu W_{2}^{i}\left(Q^{2}, \nu\right) & =x \sum_{i} e_{i}^{2} f_{i}(x)=F_{2}(x) .
\end{aligned}
$$

As equações (1.32) levam à relação de Callan-Gross [41]:

$$
F_{2}(x)=2 x F_{1}(x)
$$

A relação (1.33) foi confirmada experimentalmente, Figura (1.11), e reflete o fato de que os pártons dentro do próton possuem spin $\frac{1}{2}$; ela implica que $F_{L}(x)=0$, porém foi verificado que essa relação somente é válida em teoria de campo livre, em teoria de perturbação para teoria quântica de campos se tem desvios logarítmicos de (1.33) [42], ou seja, $F_{L}(x) \neq 0$. Nos próximos capítulos veremos que quando se leva em consideração a massa finita do alvo no DIS, a relação de Callan-Gross deixa de ser válida.

A determinação das PDFs $\left(f_{i}(x)\right)$ se dá através da análise de dados experimentais, sendo que estes precisam satisfazer certas regras de soma, por exemplo, a regra de soma do momento, considerando o nucleon composto somente por quarks:

$$
\begin{aligned}
& \int_{0}^{1} d x x \sum_{i} f_{i}(x)= \\
= & \int_{0}^{1} d x x[u(x)+\bar{u}(x)+d(x)+\bar{d}(x)+s(x)+\bar{s}(x)+\cdots]=1,
\end{aligned}
$$

onde $u(x), \bar{u}(x), d(x), \bar{d}(x), s(x)$ e $\bar{s}(x)$ representam respectivamente as distribuições de quarks up, anti-up, down, anti-down, strange e anti-strange, a soma de (1.34) se estende teoricamente a todos os sabores de quarks e anti-quarks. 


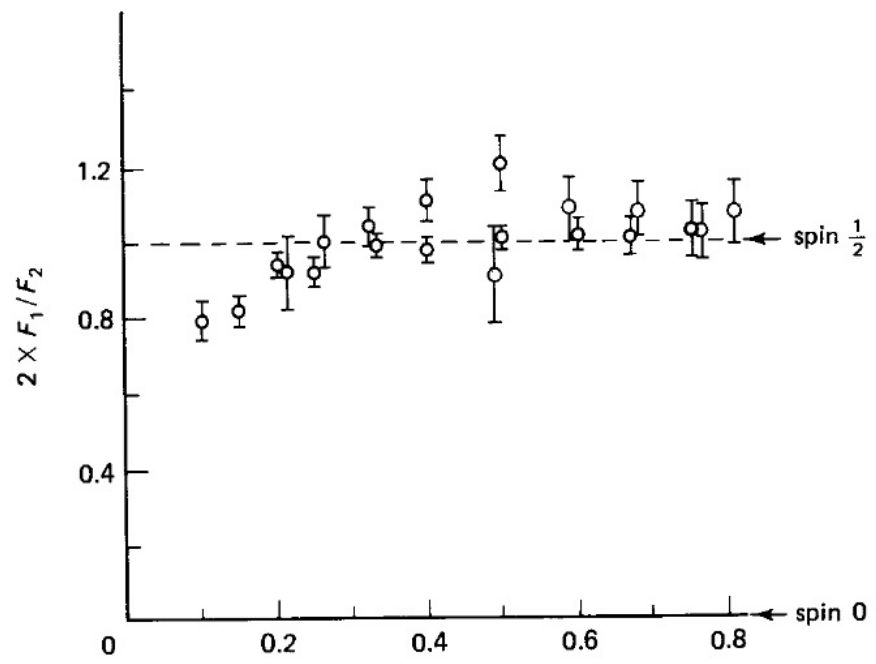

Figura 1.11: Teste da relação (1.33). Extraído de [43].

A equação (1.34) pode ser reescrita, desprezando quarks mais massivos, como:

$$
\int_{0}^{1} d x x[u(x)+\bar{u}(x)+d(x)+\bar{d}(x)+s(x)+\bar{s}(x)]=p
$$

onde $p$ é o momento carregado pelos constituintes do nucleon. Se somente existissem quarks dentro do nucleon o resultado de (1.35) teria que ser $p=1$, por conservação de momento, mas dados experimentais mostraram que $p \sim 0.54$ [44], assim aproximadamente 0.46 do momento do nucleon é carregado por constituintes eletricamente neutros, os glúons.

No modelo de quarks os mésons são compostos pelo estado ligado de 2 quarks $\left(q_{i} \bar{q}_{j}\right)$ e o nucleon sendo o estado ligado de 3 quarks $\left(q_{i} q_{j} q_{k}\right)$, e neste contexto se define os quarks de valência e do mar $q_{i}(x)=q_{i}^{v}(x)+q_{i}^{s}[10]$. O próton, pelo modelo de quarks, é o estado ligado de 2 quarks $u$ e 1 quark $d$ [31]. Então tem-se ${ }^{9}$ :

$$
\int_{0}^{1} d x u_{v}(x)=\int_{0}^{1} d x[u(x)-\bar{u}(x)]=2
$$

\footnotetext{
${ }^{9}$ Assumindo que no nucleon $q_{s}(x)=\bar{q}(x)$. Para os sabores $c, b$ e $t$, o resultado é similar ao do $s$.
} 


$$
\begin{gathered}
\int_{0}^{1} d x d_{v}(x)=\int_{0}^{1} d x[d(x)-\bar{d}(x)]=1 \\
\int_{0}^{1} d x s_{v}(x)=\int_{0}^{1} d x[s(x)-\bar{s}(x)]=0 .
\end{gathered}
$$

A produção dos outros quarks, e anti-quarks, deve-se ocorrer na forma de pares $q_{i} \bar{q}_{i}$ via glúons que são invariantes pela troca de sabor. Logo, $\int d x\left[q_{\operatorname{mar}}(x)-\bar{q}(x)\right]=0$ na produção por glúons.

Assim, pode-se escrever (1.32), considerando $s(x)=\bar{s}(x)$, para o próton como:

$$
\begin{aligned}
& F_{1}(x)=\frac{1}{2}\left\{\frac{4}{9}[u(x)+\bar{u}(x)]+\frac{1}{9}[d(x)+\bar{d}(x)+2 s(x)]\right\} \\
& F_{2}(x)=x\left\{\frac{4}{9}[u(x)+\bar{u}(x)]+\frac{1}{9}[d(x)+\bar{d}(x)+2 s(x)]\right\},
\end{aligned}
$$

onde foi usado $e_{u}=\frac{2}{3}$ e $e_{d}=e_{s}=\frac{1}{3}$.

Na Figura (1.12) está representada a forma das distribuições dos quarks, na qual verifica-se que o mar é desprezível para $x \gtrsim 0.3$ e o quark $u$ domina em grande $x$. Pode-se dizer que para $x \rightarrow 0$ a contribuição do mar deve ser levada em consideração e para $x \rightarrow 1$ a contribuição dos quarks de valência se torna dominante.

Field e Feynman em 1977 [45] foram os primeiros a argumentar que no nucleon existe uma assimetria nos quarks do mar, mais precisamente que $\bar{d}>\bar{u}$, devido ao princípio de exclusão de Pauli, pois existem mais quarks $u_{v}$ que $d_{v}$, assim aconteceria uma supressão na criação de pares $u \bar{u}$ no mar [45]. Os experimentos realizados no New Muon Collaboration (NMC) [46] e NA51 [47] trouxeram informações muito relevantes neste tópico e várias explicações para a violação na simetria do mar surgiram [48 $]^{10}$.

Em 1988 foram realizadas medidas no European Muon Collaboration (EMC) [50] da função de estrutura de spin do próton na região de pequeno $x$ até então não estudada, revelando que a contribuição que os quarks davam para o spin era pequena. No

\footnotetext{
${ }^{10} \mathrm{Um}$ estudo mais detalhado pode ser encontrado em [49].
} 


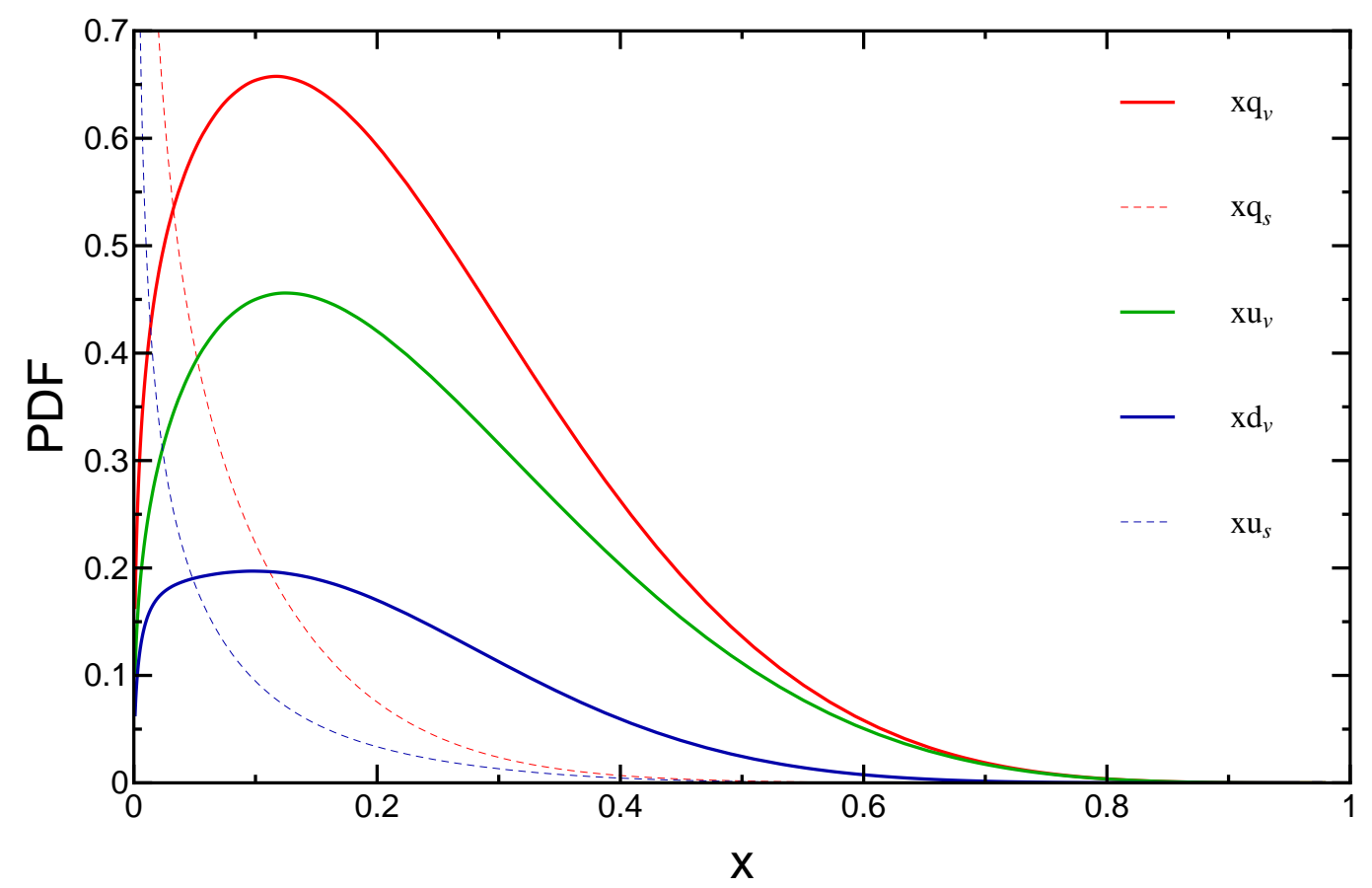

Figura 1.12: Estrutura das PDFs com relação a $x$, geradas para $Q^{2}=100 G e V^{2}$ com as parametrizações dada pela MSTW 2008 [16], na legenda $q_{i}=u_{i}+d_{i}+s_{i}$.

modelo de quarks se espera que todo spin esteja nos quarks e modelos relativísticos, como o MIT Bag Model [51], prediziam que a maior parte do spin seria dada pelos quarks e a pequena parcela restante pelos glúons e o momento angular orbital. Este problema é conhecido como spin crisis [52]. Muitos outros experimentos visando estudar este tópico foram realizados confirmando este problema [53]. Experimentos recentes verificaram que a fração que falta do spin é da ordem de $\frac{2}{3}$ [54] e existem diversas explicações para este fenômeno $[55,56]$. Acredita-se que este problema está na estrutura não perturbativa do nucleon [57], o tópico ainda continua em aberto. 


\section{Capítulo 2}

\section{Expansão do Produto de Operadores}

Neste capítulo o espalhamento elétron-nucleon profundamente inelástico é abordado de um ponto de vista mais formal, utilizando para isto a Expansão do Produto de Operadores (Operator Product Expansion - OPE) [28], que é uma maneira sistemática para extrair as regiões onde a QCD perturbativa pode ser utilizada. Mostramos um exemplo simples da aplicação da OPE no caso de campos livres. Por questão de conveniência se usa a amplitude Compton virtual, sendo que sua equivalência com o tensor hadrônico é derivada detalhadamente. Então, pela OPE, chegamos nos momentos das funções de estrutura, que mostram a quebra do scaling de Bjorken.

\section{$2.1 \quad$ OPE}

A OPE é utilizada para tratar as singularidades que aparecem quando o produto de dois operadores se dá no mesmo ponto do espaço-tempo (composite operator). Expandindo em série este produto é possível isolar esta singularidade e estudá-la em termos de operadores locais bem definidos $\left(\hat{\mathcal{O}}_{k}(x)\right)$ com coeficientes singulares $\left(\mathcal{C}_{k}(x)\right)$, 
explicitamente:

$$
\hat{A}(x) \hat{B}(y)=\sum_{k=0}^{\infty} \mathcal{C}_{k}(x-y) \hat{\mathcal{O}}_{k}\left(\frac{x+y}{2}\right)
$$

onde a singularidade que aparece no ponto $x=y$ está completamente contida em $\mathcal{C}_{k}(x-y)$, chamados de coeficientes de Wilson, e a soma é organizada em ordem decrescente de singularidade. Essa expansão foi provada por Zimmermann [58] no contexto da teoria de perturbação.

O exemplo mais simples da OPE pode ser encontrado quando se usa o teorema de Wick [59] aplicado no produto ordenado temporalmente de dois campos livres. Por exemplo, seja a corrente eletromagnética, definida como o produto normal para campos de quarks, $J^{\mu}(z)=: \bar{\Psi}(z) \gamma^{\mu} \Psi(z)$ :. Tem-se, aplicando o teorema de Wick [60]:

$$
\begin{aligned}
T\left[J^{\mu}(z) J^{\nu}(0)\right]= & -\operatorname{Tr}\left[\langle 0|T[\Psi(0) \bar{\Psi}(z)]| 0\rangle \gamma^{\mu}\langle 0|T[\Psi(z) \bar{\Psi}(0)]| 0\rangle \gamma^{\nu}\right]+ \\
& +: \bar{\Psi}(z) \gamma^{\mu}\langle 0|T[\Psi(z) \bar{\Psi}(0)]| 0\rangle \gamma^{\nu} \Psi(0):+ \\
& +: \bar{\Psi}(0) \gamma^{\nu}\langle 0|T[\Psi(0) \bar{\Psi}(z)]| 0\rangle \gamma^{\mu} \Psi(z):+ \\
& +: \bar{\Psi}(z) \gamma^{\mu} \Psi(z) \bar{\Psi}(0) \gamma^{\nu} \Psi(0):
\end{aligned}
$$

onde:

$$
i\langle 0|T[\Psi(z) \bar{\Psi}(0)]| 0\rangle=S(z)=(i \not \partial+m) \Delta(z)
$$

com $S(z)$ o propagador do quark livre e $\Delta(z)$ o propagador escalar neutro livre. A parte mais singular de $\Delta(z)$ é [60]:

$$
\Delta(z)=\frac{1}{4 \pi^{2}} \frac{1}{z^{2}-i \epsilon}
$$

A singularidade está em $z^{2} \sim 0$, ou seja, no cone-de-luz. $S(z)$ também é singular em $z^{2} \sim 0$, assim a equação (2.2) está em ordem decrescente de singularidade, sendo então uma forma restrita para a OPE genérica (2.1). 
No DIS tem-se o produto de duas correntes eletromagnéticas no mesmo ponto do espaço-tempo, assim usar a OPE no seu estudo é justificável. A aplicação da OPE neste problema forneceu a explicação através de uma teoria de campos para o DIS, e deixou claro que se necessitava de uma teoria de campos assintoticamente livres (QCD) para explicar o scaling (1.30) [61]. Para justificar o uso da OPE no DIS, observa-se que usando a definição para $W^{\alpha \beta}(p, X)(1.20)$ :

$$
\begin{aligned}
W^{\alpha \beta}(p, X) & =\frac{1}{4 \pi} \sum_{S} \sum_{X}\left\langle X\left|J^{\alpha}(0)\right| p\right\rangle^{*}\left\langle X\left|J^{\beta}(0)\right| p\right\rangle(2 \pi)^{4} \delta^{4}\left(p_{X}-p-q\right) \\
& =\frac{1}{4 \pi} \sum_{S} \sum_{X}\left\langle p\left|J^{\alpha}(0)\right| X\right\rangle\left\langle X\left|J^{\beta}(0)\right| p\right\rangle(2 \pi)^{4} \delta^{4}\left(p_{X}-p-q\right) \\
& =\frac{1}{4 \pi} \sum_{S} \sum_{X} \int d^{4} z e^{-i\left(p_{X}-p-q\right) \cdot z}\left\langle p\left|e^{-i \hat{p} \cdot z} J^{\alpha}(z) e^{i \hat{p} \cdot z}\right| X\right\rangle\left\langle X\left|J^{\beta}(0)\right| p\right\rangle \\
& =\frac{1}{4 \pi} \sum_{S} \sum_{X} \int d^{4} z e^{i q \cdot z}\left\langle p\left|J^{\alpha}(z)\right| X\right\rangle\left\langle X\left|J^{\beta}(0)\right| p\right\rangle \\
& =\frac{1}{4 \pi} \sum_{S} \int d^{4} z e^{i q \cdot z}\left\langle p\left|J^{\alpha}(z) J^{\beta}(0)\right| p\right\rangle \\
& =\frac{1}{4 \pi} \sum_{S} \int d^{4} z e^{i q \cdot z}\left\langle p\left|\left[J^{\alpha}(z), J^{\beta}(0)\right]\right| p\right\rangle .
\end{aligned}
$$

O último passo da equação (2.5) é possível pois:

$$
\begin{aligned}
& \sum_{S} \int d^{4} z e^{i q \cdot z}\left\langle p\left|J^{\beta}(0) J^{\alpha}(z)\right| p\right\rangle= \\
= & \sum_{S} \sum_{X} \int d^{4} z e^{i q \cdot z}\left\langle p\left|J^{\beta}(0)\right| X\right\rangle\left\langle X\left|J^{\alpha}(z)\right| p\right\rangle \\
= & \sum_{S} \sum_{X} \int d^{4} z e^{i\left(q+p_{X}-p\right) \cdot z}\left\langle p\left|J^{\beta}(0)\right| X\right\rangle\left\langle X\left|J^{\alpha}(0)\right| p\right\rangle
\end{aligned}
$$




$$
=\sum_{S} \sum_{X}(2 \pi)^{4} \delta^{4}\left(p_{X}-p+q\right)\left\langle p\left|J^{\beta}(0)\right| X\right\rangle\left\langle X\left|J^{\alpha}(0)\right| p\right\rangle=0 .
$$

O anulamento em (2.6) é causado pela função $\delta$ que, no sistema de laboratório, leva a $p_{X}=p-q \rightarrow E_{X}=m_{p}-\left(E-E^{\prime}\right)$ que será $<m_{p}$, pois $E^{\prime}<E$. Mas para que o estado $|X\rangle$ seja físico é preciso $E_{X} \geqslant m_{p}$, portanto este estado não existirá.

Como o comutador anula-se para $z^{2}<0$ devido a causalidade ${ }^{1}$, a integral em $(2.5)$ somente tem contribuição para $z_{\mu}$ tipo temporal:

$$
W^{\alpha \beta}(p, X)=\frac{1}{4 \pi} \sum_{S} \int_{z^{2} \geq 0} d^{4} z e^{i q \cdot z}\left\langle p\left|\left[J^{\alpha}(z), J^{\beta}(0)\right]\right| p\right\rangle .
$$

No sistema de repouso do nucleon, com a componente espacial da virtualidade do fóton $\left(q_{i}\right)$ paralela a direção do eixo $z$, temos:

$$
p_{\mu}=\left(m_{p}, 0,0,0\right), \quad q_{\mu}=\left(q_{0}, 0,0, q_{3}\right)=\left(\frac{\nu}{m_{p}}, 0,0, \sqrt{\frac{\nu^{2}}{m_{p}^{2}}+Q^{2}}\right)
$$

onde $\nu=p \cdot q=q_{0} m_{p}$ e $Q^{2}=-q^{2}=q_{3}^{2}-q_{0}^{2}=q_{3}^{2}-\frac{\nu^{2}}{m_{p}^{2}}$. Assim no limite de Bjorken, o argumento da exponencial em (2.7) fica:

$$
\begin{aligned}
e^{i q \cdot z} & =\exp \left[i\left(q_{0} z^{0}-q_{3} z^{3}\right)\right]=\exp \left[i\left(\frac{\nu}{m_{p}} z^{0}-\sqrt{\frac{\nu^{2}}{m_{p}^{2}}+Q^{2}} z^{3}\right)\right] \\
& \approx \exp \left[i \frac{\nu}{m_{p}}\left(z^{0}-z^{3}-\frac{Q^{2} m_{p}^{2}}{2 \nu^{2}} z^{3}\right)\right] \sim \exp \left[i \frac{\nu}{m_{p}}\left(z^{0}-z^{3}\right)\right] .
\end{aligned}
$$

Se $\left|z_{0}-z_{3}\right| \gg \frac{m_{p}}{\nu}$, a sua contribuição média para (2.7) será nula e se $\left|z_{0}-z_{3}\right| \ll \frac{m_{p}}{\nu}$ ou $\left|z_{0}-z_{3}\right| \propto$ constante levará a $\exp (i \infty)$ (no limite de Bjorken). Assim, para termos $W^{\alpha \beta}(p, X)$ físico e não nulo, $\left|z_{0}-z_{3}\right| \sim \mathcal{O}\left(m_{p} / \nu\right)$, e $z_{\mu}$ irá contribuir na integral

\footnotetext{
${ }^{1} \mathrm{O}$ comutador é proporcional a função de Green (propagadores da teoria), que precisa ser nulo para distâncias tipo espaciais. Esta propriedade leva a conhecida condição de microcausalidade: perturbações não podem se propagar com velocidades superluminais [62].
} 
somente quando:

$$
z^{2}=z_{0}^{2}-z_{i}^{2}=z_{0}^{2}-z_{1}^{2}-z_{2}^{2}-z_{3}^{2} \sim \mathcal{O}\left(\frac{m_{p}}{\nu}\right)-z_{1}^{2}-z_{2}^{2}
$$

portanto $z^{2} \leq \mathcal{O}\left(\frac{m_{p}}{\nu}\right)$

Juntando o argumento de (2.10) e o fato que o comutador se anula para $z^{2}<0$, conclui-se que o tensor hadrônico é controlado pelos elementos de matriz para $z$ no cone de luz, $z^{2} \sim 0$.

\subsection{Momento das funções de estrutura}

No final da Subseção 1.2.2 foi mencionado que pode-se interpretar as funções de estrutura através da amplitude do espalhamento Compton virtual, Figura (1.6). Tal amplitude é dada por:

$$
T_{\mu \nu}=i \int d^{4} z e^{i q \cdot z}\left\langle p\left|T\left[J_{\mu}(z) J_{\nu}(0)\right]\right| p\right\rangle,
$$

onde a soma dos spins é subentendida.

A relação entre (2.11) e (1.20) é dada por [60]:

$$
W_{\mu \nu}\left(x, Q^{2}\right)=\frac{1}{\pi} A b s\left[T_{\mu \nu}\left(x, Q^{2}\right)\right]
$$

sendo $A b s\left[T_{\mu \nu}\right]$ a parte absortiva da amplitude, ou seja, a descontinuidade de $T_{\mu \nu}$ através do corte sobre a linha $q_{0}>0$ no plano complexo de $q_{0}$ :

$$
A b s\left[T_{\mu \nu}\right]=\frac{1}{2 i}\left[T_{\mu \nu}\left(q_{0}+i \epsilon\right)-T_{\mu \nu}\left(q_{0}-i \epsilon\right)\right] .
$$

A validade da relação (2.12) pode ser verificada a seguir:

$$
T_{\mu \nu}=i \int d^{4} z e^{i q \cdot z}\left\langle p\left|T\left[J_{\mu}(z) J_{\nu}(0)\right]\right| p\right\rangle
$$




$$
\begin{aligned}
& =i \int d^{4} z e^{i q \cdot z}\left\langle p\left|\left\{\theta\left(z_{0}\right) J_{\mu}(z) J_{\nu}(0)+\theta\left(-z_{0}\right) J_{\nu}(0) J_{\mu}(z)\right\}\right| p\right\rangle \\
& =i \int d^{4} z e^{i q \cdot z} \theta\left(z_{0}\right) \sum_{X}\left\langle p\left|e^{i \hat{p} \cdot z} J_{\mu}(0) e^{-i \hat{p} \cdot z}\right| X\right\rangle\left\langle X\left|J_{\nu}(0)\right| p\right\rangle+ \\
& +\quad i \int d^{4} z e^{i q \cdot z} \theta\left(-z_{0}\right) \sum_{X}\left\langle p\left|J_{\nu}(0)\right| X\right\rangle\left\langle X\left|e^{i \hat{p} \cdot z} J_{\mu}(0) e^{-i \hat{p} \cdot z}\right| p\right\rangle \\
& =i \int d^{3} z e^{i\left(-q_{i}-p_{i}+p_{X_{i}}\right) z^{i}} \int d z_{0} e^{i\left(q_{0}+p_{0}-p_{X_{0}}\right) z^{0}} \theta\left(z_{0}\right) \times \\
& \times \sum_{X}\left\langle p\left|J_{\mu}(0)\right| X\right\rangle\left\langle X\left|J_{\nu}(0)\right| p\right\rangle+ \\
& +i \int d^{3} z e^{i\left(-q_{i}+p_{i}-p_{X_{i}}\right) z^{i}} \int d z_{0} e^{i\left(q_{0}-p_{0}+p_{X_{0}}\right) z^{0}} \theta\left(-z_{0}\right) \times \\
& \times \sum_{X}\left\langle p\left|J_{\nu}(0)\right| X\right\rangle\left\langle X\left|J_{\mu}(0)\right| p\right\rangle \\
& =\frac{(2 \pi)^{3} \delta^{3}\left(p_{X_{i}}-q_{i}-p_{i}\right)}{p_{X_{0}}-q_{0}-p_{0}} \sum_{X}\left\langle p\left|J_{\mu}(0)\right| X\right\rangle\left\langle X\left|J_{\nu}(0)\right| p\right\rangle+ \\
& +\frac{(2 \pi)^{3} \delta^{3}\left(p_{X_{i}}+q_{i}-p_{i}\right)}{p_{X_{0}}+q_{0}-p_{0}} \sum_{X}\left\langle p\left|J_{\nu}(0)\right| X\right\rangle\left\langle X\left|J_{\mu}(0)\right| p\right\rangle,
\end{aligned}
$$

onde $\theta\left(z_{0}\right)$ é a função de Heaviside (função degrau).

O segundo termo de (2.14) não possui descontinuidade, pois $p_{X_{0}}+q_{0}-p_{0} \neq 0$ para $q_{0}>0$, assim somente o primeiro termo contribui para a descontinuidade de $T_{\mu \nu}$ no plano complexo de $q_{0}$. Então, com (2.14) e (2.13):

$$
\begin{aligned}
A b s\left[T_{\mu \nu}\right] & =\frac{1}{2 i}\left[T_{\mu \nu}\left(q_{0}+i \epsilon\right)-T_{\mu \nu}\left(q_{0}-i \epsilon\right)\right] \\
& =\frac{1}{2 i}\left(\frac{1}{p_{X_{0}}-q_{0}-p_{0}-i \epsilon}-\frac{1}{p_{X_{0}}-q_{0}-p_{0}+i \epsilon}\right) \times \\
& \times(2 \pi)^{3} \delta^{3}\left(p_{X_{i}}-q_{i}-p_{i}\right) \sum_{X}\left\langle p\left|J_{\mu}(0)\right| X\right\rangle\left\langle X\left|J_{\nu}(0)\right| p\right\rangle
\end{aligned}
$$




$$
\begin{aligned}
& =\frac{1}{2 i}\left[\left(\frac{\mathcal{P}}{p_{X_{0}}-q_{0}-p_{0}}+i \pi \delta\left(p_{X_{0}}-q_{0}-p_{0}\right)\right)-\right. \\
& \left.\quad-\left(\frac{\mathcal{P}}{p_{X_{0}}-q_{0}-p_{0}}-i \pi \delta\left(p_{X_{0}}-q_{0}-p_{0}\right)\right)\right] \times \\
& \times \quad(2 \pi)^{3} \delta^{3}\left(p_{X_{i}}-q_{i}-p_{i}\right) \sum_{X}\left\langle p\left|J_{\mu}(0)\right| X\right\rangle\left\langle X\left|J_{\nu}(0)\right| p\right\rangle \\
& =\pi(2 \pi)^{3} \delta^{4}\left(p_{X}-q-p\right) \sum_{X}\left\langle p\left|J_{\mu}(0)\right| X\right\rangle\left\langle X\left|J_{\nu}(0)\right| p\right\rangle=\pi W_{\mu \nu},
\end{aligned}
$$

onde $\mathcal{P}$ denota a parte principal ${ }^{2}$.

O uso de $T_{\mu \nu}$ é por conveniência, pois devido a algumas propriedades tem-se uma maior facilidade de resolver as integrações. Entre as propriedades, temos a simetria de cruzamento [60, 64]:

$$
T_{\mu \nu}\left(x, Q^{2}\right)=T_{\nu \mu}\left(-x, Q^{2}\right)
$$

e a função é analítica, $T_{\mu \nu}\left(x^{*}, Q^{2}\right)=T_{\mu \nu}^{*}\left(x, Q^{2}\right)$, menos em $\omega \geq 1$ e $\omega \leq-1[60,64]$.

Além da relação (2.12), existe uma relação integral muito útil entre a amplitude $T$ e o tensor $W$. Como foi mencionado, $T$ possui pólos em $\omega= \pm 1$ e cortes de ramo (branch cuts) em $\omega \geq 1$ e $\omega \leq-1$, assim integrando pelo caminho $\mathcal{C}$, mostrado na Figura (2.1), tem-se:

$$
\begin{aligned}
\oint_{\mathcal{C}} \omega^{-n} T_{\mu \nu} d \omega & =\int_{1_{\text {acima }}}^{\infty} \omega^{-n} T_{\mu \nu} d \omega+\int_{-\infty_{\text {acima }}}^{-1} \omega^{-n} T_{\mu \nu} d \omega+ \\
& +\int_{-1_{\text {abaixo }}}^{-\infty} \omega^{-n} T_{\mu \nu} d \omega+\int_{\infty_{\text {abaixo }}}^{1} \omega^{-n} T_{\mu \nu} d \omega
\end{aligned}
$$

assim para $n$ par e com (2.16), a parte real se cancela e a imaginária se soma:

$$
\frac{1}{2 \pi i} \oint_{\mathcal{C}} \omega^{-n} T_{\mu \nu} d \omega=\frac{2}{\pi} \int_{1}^{\infty} \omega^{-n} A b s\left[T_{\mu \nu}\right] d \omega=2 \int_{0}^{1} x^{n-2} W_{\mu \nu} d x
$$

\footnotetext{
${ }^{2} \mathrm{~A}$ parte principal, $\mathcal{P}$, de uma função consiste nos termos com potência negativa da série de Laurent no respectivo pólo da função [63].
} 


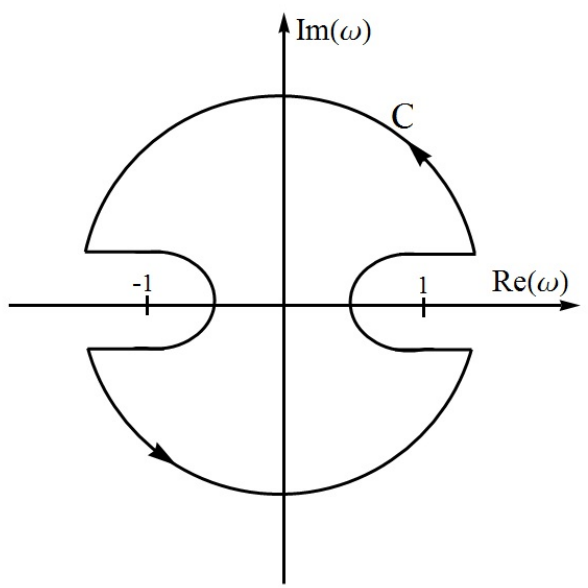

Figura 2.1: Caminho $\mathcal{C}$ de integração usado em (2.17).

Vale ressaltar uma integral muito útil [60]:

$$
\frac{1}{2 \pi i} \oint_{\mathcal{C}} \omega^{m-n} d \omega=\delta_{m, n-1}
$$

A OPE dada pela equação (2.1) fornece informações da região de distâncias curtas, porém no caso do DIS se quer informações da região no cone de luz. A generalização para a expansão no cone de luz é dada por [58, 65]:

$$
J_{\mu}(z) J_{\nu}(0)=\sum_{n=0}^{\infty} i^{n-1} C_{n}^{(k)}\left(z^{2}-i \epsilon\right) z^{\mu_{1}} z^{\mu_{2}} \cdots z^{\mu_{n}} \mathcal{O}_{\mu_{1} \mu_{2} \ldots \mu_{n}}^{(k)}(0)
$$

onde $z^{2} \sim 0$, e o índice $k$ distingue os diferentes tipos de operadores locais que possuem os mesmos números quânticos, como o produto das correntes para cada $n$ na expansão.

Uma forma de organizar os vários operadores com os mesmos números quânticos é pelo seu twist [66], pois quanto menor o twist um operador possuir, mais singular é o seu coeficiente. Uma análise dimensional de massa dos coeficientes de Wilson em (2.20) mostra que:

$$
\left[C_{n}^{(k)}\left(z^{2}-i \epsilon\right)\right]=2 d_{j}+n-d_{0}
$$


onde $d_{j}$ é a dimensão de massa da corrente, $-n$ a dimensão de massa do produto $z^{\mu_{1}} z^{\mu_{2}} \cdots z^{\mu_{n}}$ e $d_{0}$ a dimensão de massa do operador $\mathcal{O}$. O twist do operador $\mathcal{O}$ é definido como:

$$
\tau=d_{0}-n
$$

O operador mais simples é $\bar{\Psi} \gamma_{\mu} \Psi$, que possui $n=1$ e $[\Psi]=\frac{3}{2}$ assim seu twist, dado pela equação (2.22), é $\tau=2$, ou seja, o menor twist que um operador pode ter. Mas pode-se adicionar derivadas a este simples operador e preservar o seu twist, por exemplo [67]:

$$
\begin{aligned}
& \mathcal{O}_{\mu_{1} \mu_{2} \ldots \mu_{n}}^{(k)}=i^{n-1} S \bar{\Psi} \gamma_{\mu_{1}} D_{\mu_{2}} \cdots D_{\mu_{n}} \Psi \\
& \mathcal{O}_{\mu_{1} \mu_{2} \ldots \mu_{n}}^{(k)}=i^{n-1} S \bar{\Psi} \gamma_{\mu_{1}} D_{\mu_{2}} \cdots D_{\mu_{n}} \frac{\lambda^{j}}{2} \Psi
\end{aligned}
$$

onde $D_{\mu}=\partial_{\mu}-i g A_{\mu}^{a} T^{a}$ é a derivada covariante e $T^{a}$ são matrizes do grupo de geradores na representação de férmions [62], $S$ denota a simetrização nos índices de Lorentz, o fator $i^{n-1}$ foi adicionado por conveniência e $\lambda^{j}$ são as matrizes de GellMann. O operador (2.23a) não faz distinção entre os quarks e por isto recebe o nome de operador singleto de sabor e o operador (2.23b), por incluir o termo $\lambda^{j}$ não é invariantes sobre troca de sabor e é chamado de operador não singleto.

A forma mais geral para o produto ordenado temporalmente de duas correntes eletromagnéticas, invariante de Lorentz e com corrente conservada, possui a seguinte forma [68]:

$$
\begin{aligned}
T\left[J_{\mu}(z) J_{\nu}(0)\right]= & -\left(g_{\mu \nu} \square-\partial_{\mu} \partial_{\nu}\right) \mathcal{O}_{L}(z, 0)+ \\
& +\left(g_{\mu \lambda} \partial_{\rho} \partial_{\nu}+g_{\rho \nu} \partial_{\mu} \partial_{\lambda}-g_{\mu \lambda} g_{\rho \nu} \square-g_{\mu \nu} \partial_{\rho} \partial_{\lambda}\right) \mathcal{O}_{2}^{\lambda \rho}(z, 0),
\end{aligned}
$$

onde a parte antissimétrica foi desprezada, pois ela não contribui quando os quarks 
não são polarizados [60].

Aplicando a OPE no cone de luz, (2.20), tem-se:

$$
\begin{aligned}
& \mathcal{O}_{L}(z, 0)=\sum_{k, n} i^{n-1} C_{L, n}^{(k)}\left(z^{2}-i \epsilon\right) z^{\mu_{1}} z^{\mu_{2}} \cdots z^{\mu_{n}} \mathcal{O}_{L, \mu_{1} \mu_{2} \ldots \mu_{n}}^{(k)}(0) \\
& \mathcal{O}_{2}^{\lambda \rho}(z, 0)=\sum_{k, n} i^{n-1} C_{2, n}^{(k)}\left(z^{2}-i \epsilon\right) z^{\mu_{1}} z^{\mu_{2}} \cdots z^{\mu_{n}} \mathcal{O}_{2, \mu_{1} \mu_{2} \ldots \mu_{n}}^{(k) \lambda \rho}(0) .
\end{aligned}
$$

Utilizando as equações (2.24) e (2.25) em (2.11) chega-se em:

$$
\begin{aligned}
T_{\mu \nu} & =i \int d^{4} z e^{i q \cdot z}\left\langle p\left|T\left[J_{\mu}(z) J_{\nu}(0)\right]\right| p\right\rangle \\
& =\sum_{k, n} i^{n}\left\langle p\left|\mathcal{O}_{L, \mu_{1} \mu_{2} \ldots \mu_{n}}^{(k)}(0)\right| p\right\rangle \int d^{4} z z^{\mu_{1}} \ldots z^{\mu_{n}} C_{L, n}^{(k)}\left(z^{2}-i \epsilon\right)\left(\partial_{\mu} \partial_{\nu}-g_{\mu \nu} \square\right) e^{i q \cdot z} \\
& +\sum_{k, n} i^{n}\left\langle p\left|\mathcal{O}_{2, \mu_{1} \mu_{2} \ldots \mu_{n}}^{(k) \lambda \rho}(0)\right| p\right\rangle \int d^{4} z z^{\mu_{1}} \ldots z^{\mu_{n}} C_{2, n}^{(k)}\left(z^{2}-i \epsilon\right) \\
& \times\left(g_{\mu \lambda} \partial_{\rho} \partial_{\nu}+g_{\rho \nu} \partial_{\mu} \partial_{\lambda}-g_{\mu \lambda} g_{\rho \nu} \square-g_{\mu \nu} \partial_{\rho} \partial_{\lambda}\right) e^{i q \cdot z} \\
& =-\left(q_{\mu} q_{\nu}-q^{2} g_{\mu \nu}\right) \sum_{k, n} i^{n}\left\langle p\left|\mathcal{O}_{L, \mu_{1} \mu_{2} \ldots \mu_{n}}^{(k)}(0)\right| p\right\rangle \int d^{4} z z^{\mu_{1}} \ldots z^{\mu_{n}} \\
& \times C_{L, n}^{(k)}\left(z^{2}-i \epsilon\right) e^{i q \cdot z}-\left(g_{\mu \lambda} q_{\rho} q_{\nu}+g_{\rho \nu} q_{\mu} q_{\lambda}-q^{2} g_{\mu \lambda} g_{\rho \nu}-g_{\mu \nu} q_{\rho} q_{\lambda}\right) \\
& \times \sum_{k, n} i^{n}\left\langle p\left|\mathcal{O}_{2, \mu_{1} \mu_{2} \ldots \mu_{n}}^{(k) \lambda \rho}(0)\right| p\right\rangle \int d^{4} z z^{\mu_{1}} \ldots z^{\mu_{n}} C_{2, n}^{(k)}\left(z^{2}-i \epsilon\right) e^{i q \cdot z} .
\end{aligned}
$$

Por conveniência faz-se a substituição:

$$
z^{\mu_{1}} \cdots z^{\mu_{n}} e^{i q \cdot z}=(-i)^{n} \frac{\partial}{\partial q^{\mu_{1}}} \cdots \frac{\partial}{\partial q^{\mu_{n}}} e^{i q \cdot z}=(-2 i)^{n} q^{\mu_{1}} \cdots q^{\mu_{n}}\left(\frac{\partial}{\partial q^{2}}\right)^{n} e^{i q \cdot z}
$$

Os elementos de matriz dos operadores $\mathcal{O}$ só podem ser função do momento $p$; assim, eles precisam possuir a forma tensorial [60, 64, 69]:

$$
\left\langle p\left|\mathcal{O}_{L, \mu_{1} \mu_{2} \ldots \mu_{n}}^{(k)}(0)\right| p\right\rangle=\mathcal{A}_{L, n}^{(k)} p_{\mu_{1}} \cdots p_{\mu_{n}}+\text { termos com } g_{\mu_{i} \mu_{j}}
$$




$$
\left\langle p\left|\mathcal{O}_{2, \mu_{1} \mu_{2} \ldots \mu_{n}}^{(k) \lambda \rho}(0)\right| p\right\rangle=\mathcal{A}_{2, n+2}^{(k)} p^{\lambda} p^{\rho} p_{\mu_{1}} \cdots p_{\mu_{n}}+\text { termos com } g_{\mu_{i} \mu_{j}},
$$

onde "termos com $g_{\mu_{i} \mu_{j}}$ " podem ser da forma $p^{2} g_{\mu_{1} \mu_{2}} p_{\mu_{3}} \cdots p_{\mu_{n}}$ e com dois ou mais $g_{\mu_{i} \mu_{j}}$; porém, estes termos são irrelevantes, no limite de Bjorken, quando comparados ao termo sem $g_{\mu_{i} \mu_{j}}$. No capítulo seguinte iremos considerar estes termos e obteremos assim uma correção nas funções de estrutura.

Substituindo as equações (2.27) e (2.28) em (2.26) tem-se:

$$
\begin{aligned}
T_{\mu \nu} & =\left(q_{\mu} q_{\nu}-q^{2} g_{\mu \nu}\right) \sum_{k, n} \mathcal{A}_{L, n}^{(k)} p_{\mu_{1}} \cdots p_{\mu_{n}} \int d^{4} z(-2)^{n} q^{\mu_{1}} \cdots q^{\mu_{n}}\left(\frac{\partial}{\partial q^{2}}\right)^{n} \times \\
& \times C_{L, n}^{(k)}\left(z^{2}-i \epsilon\right) e^{i q \cdot z}+\left(g_{\mu \lambda} q_{\rho} q_{\nu}+g_{\rho \nu} q_{\mu} q_{\lambda}-q^{2} g_{\mu \lambda} g_{\rho \nu}-g_{\mu \nu} q_{\rho} q_{\lambda}\right) \sum_{k, n} \mathcal{A}_{2, n+2}^{(k)} \times \\
& \times p^{\lambda} p^{\rho} p_{\mu_{1}} \cdots p_{\mu_{n}} \int d^{4} z(-2)^{n} q^{\mu_{1}} \cdots q^{\mu_{n}}\left(\frac{\partial}{\partial q^{2}}\right)^{n} C_{2, n}^{(k)}\left(z^{2}-i \epsilon\right) e^{i q \cdot z} \\
& =\left(q_{\mu} q_{\nu}-q^{2} g_{\mu \nu}\right) \sum_{k, n} \mathcal{A}_{L, n}^{(k)}(-2 p \cdot q)^{n}\left(\frac{\partial}{\partial q^{2}}\right)^{n} \int d^{4} z C_{L, n}^{(k)}\left(z^{2}-i \epsilon\right) e^{i q \cdot z}+ \\
& +\left[p \cdot q p_{\mu} q_{\nu}+p \cdot q q_{\mu} p_{\nu}-q^{2} p_{\mu} p_{\nu}-(p \cdot q)^{2} g_{\mu \nu}\right] \sum_{k, n} \mathcal{A}_{2, n+2}^{(k)}(-2 p \cdot q)^{n} \times \\
& \times\left(\frac{\partial}{\partial q^{2}}\right)^{n} \int d^{4} z C_{2, n}^{(k)}\left(z^{2}-i \epsilon\right) e^{i q \cdot z} \\
& =-e_{\mu \nu} \sum_{k, n} \omega^{n}\left(q^{2}\right)^{n+1} \mathcal{A}_{L, n}^{(k)}\left(\frac{\partial}{\partial q^{2}}\right)^{n} \int d^{4} z C_{L, n}^{(k)}\left(z^{2}-i \epsilon\right) e^{i q \cdot z}+ \\
& +d_{\mu \nu} \sum_{k, n} \omega^{n+2} \frac{\left(q^{2}\right)^{n+2}}{4} \mathcal{A}_{2, n+2}^{(k)}\left(\frac{\partial}{\partial q^{2}}\right)^{n} \int d^{4} z C_{2, n}^{(k)}\left(z^{2}-i \epsilon\right) e^{i q \cdot z}
\end{aligned}
$$

onde $e_{\mu \nu}=g_{\mu \nu}-\frac{q_{\mu} q_{\nu}}{q^{2}}$ e $d_{\mu \nu}=\frac{p_{\mu} q_{\nu}+q_{\mu} p_{\nu}}{p \cdot q}-\frac{q^{2}}{(p \cdot q)^{2}} p_{\mu} p_{\nu}-g_{\mu \nu}$.

Para o caso de campos livres, os coeficientes de Wilson são bem conhecidos [64, 69]:

$$
C_{L}\left(z^{2}-i \epsilon\right) \propto \frac{1}{\left(z^{2}-i \epsilon\right)^{2}} \rightarrow \int d^{4} z \frac{e^{-i q \cdot z}}{\left(z^{2}-i \epsilon\right)^{2}} \propto-\log \left(q^{2}+i \epsilon\right)
$$




$$
C_{2}\left(z^{2}-i \epsilon\right) \propto \frac{1}{z^{2}-i \epsilon} \rightarrow \int d^{4} z \frac{e^{-i q \cdot z}}{z^{2}-i \epsilon} \propto \frac{1}{q^{2}+i \epsilon}
$$

Assim define-se uma forma geral para os coeficientes de Wilson [69]:

$$
\begin{aligned}
& C_{L, n}^{(k)}\left(q^{2}\right)=-\frac{\left(q^{2}\right)^{n+1}}{2}\left(\frac{\partial}{\partial q^{2}}\right)^{n} \int d^{4} z C_{L, n}^{(k)}\left(z^{2}-i \epsilon\right) e^{i q \cdot z} \\
& C_{2, n+2}^{(k)}\left(q^{2}\right)=\frac{\left(q^{2}\right)^{n+2}}{8}\left(\frac{\partial}{\partial q^{2}}\right)^{n} \int d^{4} z C_{2, n}^{(k)}\left(z^{2}-i \epsilon\right) e^{i q \cdot z}
\end{aligned}
$$

Então:

$$
T_{\mu \nu}=2 \sum_{k, n}\left[e_{\mu \nu} \omega^{n} \mathcal{A}_{L, n}^{(k)} C_{L, n}^{(k)}\left(q^{2}\right)+d_{\mu \nu} \omega^{n+2} \mathcal{A}_{2, n+2}^{(k)} C_{2, n+2}^{(k)}\left(q^{2}\right)\right]
$$

lembrando que, pela simetria de cruzamento (2.16), $n$ é par.

Pode-se escrever o tensor hadrônico, dado por (1.22), no limite de Bjorken (1.30) como:

$$
\begin{aligned}
\frac{W_{\mu \nu}}{2 m_{p}}= & {\left[\frac{q_{\mu} q_{\nu}}{q^{2}}-g_{\mu \nu}\right] W_{1}\left(Q^{2}, \nu\right)+\left[\left(p_{\mu}-\frac{p \cdot q}{q^{2}} q_{\mu}\right)\left(p_{\nu}-\frac{p \cdot q}{q^{2}} q_{\nu}\right)\right] \frac{W_{2}\left(Q^{2}, \nu\right)}{m_{p}^{2}} } \\
W_{\mu \nu}= & -\left[g_{\mu \nu}-\frac{q_{\mu} q_{\nu}}{q^{2}}\right] 2 F_{1}(x) \\
& +\left[p_{\mu} p_{\nu}-\frac{p \cdot q}{q^{2}}\left(p_{\mu} q_{\nu}+p_{\nu} q_{\mu}\right)+\frac{(p \cdot q)^{2}}{q^{4}} q_{\mu} q_{\nu}\right] \frac{2 F_{2}(x)}{\nu m_{p}} \\
= & -e_{\mu \nu} 2 F_{1}(x) \\
& +\left[-\frac{q^{2}}{(p \cdot q)^{2}} p_{\mu} p_{\nu}+\frac{\left(p_{\mu} q_{\nu}+p_{\nu} q_{\mu}\right)}{p \cdot q}-g_{\mu \nu}+g_{\mu \nu}-\frac{q_{\mu} q_{\nu}}{q^{2}}\right] \frac{(p \cdot q)^{2}}{-q^{2}} \frac{2 F_{2}(x)}{p \cdot q} \\
= & -2 e_{\mu \nu} F_{1}(x)+\left[d_{\mu \nu}+e_{\mu \nu}\right] \frac{F_{2}(x)}{x} \\
= & \frac{e_{\mu \nu}}{x} F_{L}(x)+\frac{d_{\mu \nu}}{x} F_{2}(x),
\end{aligned}
$$


onde $F_{L}=F_{2}-2 x F_{1}$ é a função de estrutura longitudinal.

Substituindo (2.32) e (2.33) em (2.18) chega-se:

$$
\begin{aligned}
& M_{L}^{(n)}\left(Q^{2}\right)=\int_{0}^{1} d x x^{n-2} F_{L}\left(x, Q^{2}\right)=\sum_{k} \mathcal{A}_{L, n}^{(k)} C_{L, n}^{(k)}\left(Q^{2}\right) ; \\
& M_{2}^{(n)}\left(Q^{2}\right)=\int_{0}^{1} d x x^{n-2} F_{2}\left(x, Q^{2}\right)=\sum_{k} \mathcal{A}_{2, n}^{(k)} C_{2, n}^{(k)}\left(Q^{2}\right) .
\end{aligned}
$$

As equações em (2.34) são chamadas de momento das funções de estrutura e com elas podemos fazer predições da dependência com $Q^{2}$. Os coeficientes $C_{L, n}^{(k)}\left(Q^{2}\right)$ e $C_{2, n}^{(k)}\left(Q^{2}\right)$ são sensíveis à física de curtas distâncias no cone-de-luz, calculáveis através da QCD perturbativa (para isto utiliza-se o grupo de renormalização $[60,64,67]$ ), enquanto $\mathcal{A}_{L, n}^{(k)}$ e $\mathcal{A}_{2, n}^{(k)}$ estão relacionadas às distâncias longas e devem ser determinadas experimentalmente.

As relações (2.34) também recebem o nome de momento de Cornwall-Norton [70] e generalizando:

$$
M_{i}^{(n)}\left(Q^{2}\right)= \begin{cases}\int_{0}^{1} d x x^{n-1} F_{i}\left(x, Q^{2}\right), & \text { se } i=1,3,4,5 \\ \int_{0}^{1} d x x^{n-2} F_{i}\left(x, Q^{2}\right), & \text { se } i=2, L\end{cases}
$$




\section{Capítulo 3}

\section{Correções da Massa do Alvo pela}

\section{OPE}

Na derivação das equações (2.34) foi utilizada uma aproximação para os elementos de matriz (2.28) desconsiderando termos com $g_{\mu_{i} \mu_{j}}$, pois no limite de Bjorken estes termos são desprezíveis, proporcionais a $\frac{m_{p}^{2}}{Q^{2}}$. Estas correções em potências de $Q^{2}$ aparecem por efeitos puramente cinemáticos e são chamadas de correções da massa do alvo (Target Mass Corrections - TMC). Estas contribuições cinemáticas são diferentes daquelas correções em potências de $\frac{1}{Q^{2}}$ que aparecem devido à inclusão de twists mais altos nos cálculos das funções de estrutura [27]. Estas correções de twists mais altos são dinâmicas, em oposição às correções cinemáticas vinda das TMC. Para baixo $Q^{2}$, em uma análise consistente, ambas correções devem estar presentes [71].

No limite em que $Q^{2} \gg m_{p}^{2}$ e grande massa do estado hadrônico final $\left(W^{2}=\right.$ $\left.m_{p}^{2}+Q^{2} \frac{(1-x)}{x}\right)$, região em que as TMC são desprezíveis, tem-se um sucesso considerável na descrição dos dados experimentais ${ }^{1}$. Porém, como experimentos recentes e precisos em baixo $Q^{2}$ (onde é preciso considerar as TMC) foram realizados [73], e outros já foram propostos [74], é preciso obter uma maneira consistente de tratar as funções de estrutura com as TMC.

\footnotetext{
${ }^{1}$ Veja as análises globais, [16, 72], por exemplo.
} 


\subsection{Abordagem utilizada por Georgi e Politzer}

As TMC foram consideradas primeiramente por Nachtmann [25], que ao estudar a região onde $Q^{2}$ é finito introduziu uma nova variável de scaling, definida como sendo a fração que o párton, de momento $z$, carrega do momento $p$, no cone de luz, do nucleon:

$$
\xi\left(x, Q^{2}\right) \equiv \frac{z^{0}+z^{3}}{p^{0}+p^{3}}=\frac{2 x}{1+\rho}, \quad \rho=\sqrt{1+4 \mu x^{2}} \quad \text { e } \quad \mu=\frac{m_{p}^{2}}{Q^{2}}
$$

No limite $Q^{2} \rightarrow \infty$, tem-se $\xi=x$, como pode ser facilmente verificada da definição de $\xi$ e visualizada na Figura (3.1). Um aspecto interessante da Figura (3.1) é que mesmo para $Q^{2}$ tão pequeno quanto $1 \mathrm{GeV}^{2}$, existem regiões de $x$ em que $\xi=x$, implicando que a massa do alvo é relevante para as regiões de $x$ grande, ou seja, nas proximidades do limite elástico $(x=1)$.

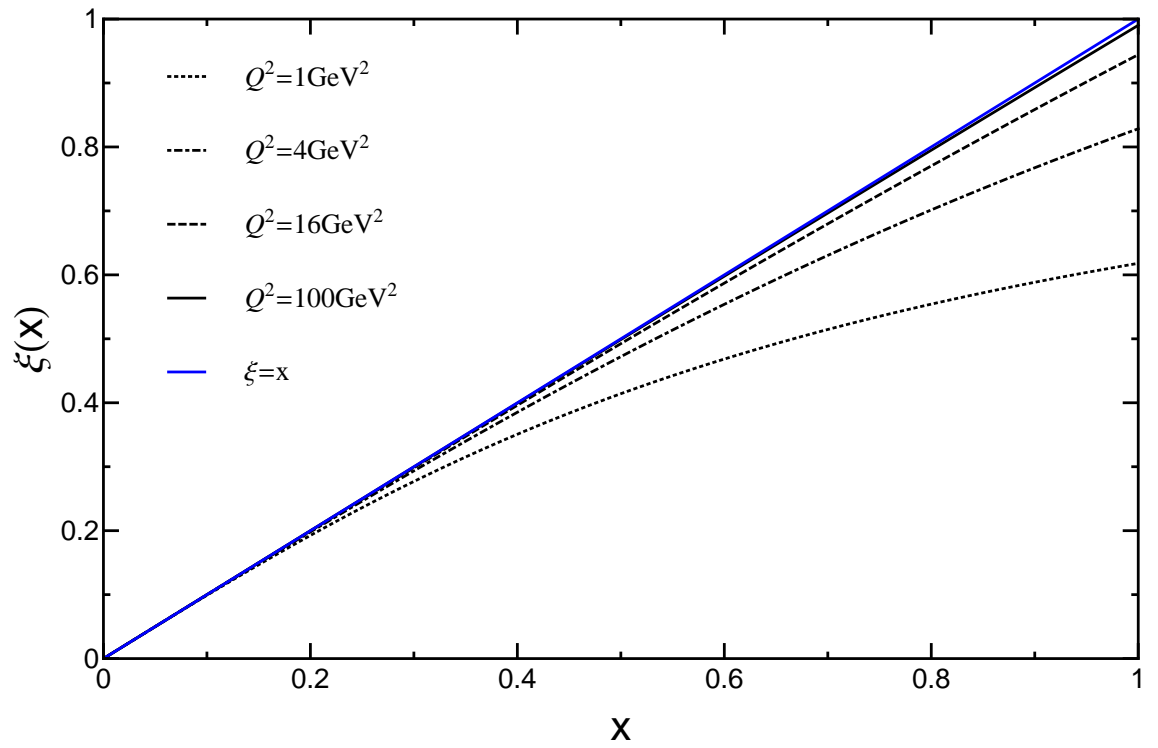

Figura 3.1: Variável de Nachtmann, equação (3.1), em função de $x$ para vários valores de $Q^{2}$ e o caso limite $\xi=x$.

A primeira análise com o intuito de incluir efeitos de $Q^{2}$ finito em QCD foi feita por Georgi e Politzer [26], a qual levou em consideração os termos com $g_{\mu_{i} \mu_{j}}$ que aparecem nos elementos de matriz dos operadores de twist 2 . Assim gera-se uma série em $\mu=\frac{m_{p}^{2}}{Q^{2}}$ 
nos momentos de Cornwall-Norton, que faz com que as funções de estrutura dependam de $x$ e $\mu$.

Seguindo o procedimento feito em [26], começamos escrevendo o tensor hadrônico da forma mais genérica possível, permitida pela invariância de Lorentz:

$$
\begin{aligned}
W^{\mu \nu} & =\frac{1}{2 \pi} \int d^{4} z e^{i q \cdot z}\left\langle p\left|\left[J^{\mu}(z), J^{\nu}(0)\right]\right| p\right\rangle \\
& =-g^{\mu \nu} W_{1}+\frac{p^{\mu} p^{\nu}}{m_{p}^{2}} W_{2}-i \epsilon^{\mu \nu \lambda \sigma} \frac{p_{\lambda} q_{\sigma}}{m_{p}^{2}} W_{3}+\frac{2 q^{\mu} q^{\nu}}{m_{p}^{2}} W_{4}+\frac{p^{\mu} q^{\nu}+p^{\nu} q^{\mu}}{m_{p}^{2}} W_{5}
\end{aligned}
$$

sendo a relação entre $W_{i}$ e $F_{i}$ dada por [75]:

$$
\begin{aligned}
& \left\{F_{1}, F_{2}, F_{L}, F_{3}, F_{4}, F_{5}\right\}= \\
& \left\{W_{1}, \frac{1}{2 \mu x} W_{2},\left(1+4 \mu x^{2}\right) \frac{1}{2 \mu x} W_{2}-2 x W_{1}, \frac{1}{\mu x} W_{3}, \frac{1}{2 \mu} W_{4}, \frac{1}{2 \mu x} W_{5}\right\},
\end{aligned}
$$

onde as $F$ são as funções de estrutura do nucleon. $F_{1}$ e $F_{2}$ são determinadas por espalhamentos de léptons carregados (produto de correntes vetoriais), $F_{3}$ pela interferência do produto de correntes vetoriais e axiais (devido à violação de paridade do tensor anti-simétrico $\epsilon^{\mu \nu \lambda \sigma}$ ), e $F_{4}$ e $F_{5}$ por espalhamentos de neutrinos. Estas últimas são suprimidas pelas massas dos léptons $\left(\frac{m_{l}^{2}}{m_{p}^{2}}\right)[75]$ e estão na análise por completeza.

\subsubsection{Momento de Cornwall-Norton com as TMC}

Seguindo [26] utilizaremos a amplitude de espalhamento Compton $T^{\mu \nu}$, sendo a relação entre o tensor hadrônico e a amplitude Compton dada por (2.12). $T^{\mu \nu}$ pode ser expressa na OPE como [27]:

$$
\begin{aligned}
T^{\mu \nu} & =i \int d^{4} z e^{i q \cdot z}\left\langle p\left|T\left[J^{\mu}(z) J^{\nu}(0)\right]\right| p\right\rangle \\
& =\sum_{i, \tau, n} c_{i, \mu_{1} \ldots \mu_{n}}^{\tau, \mu \nu}(q)\left\langle p\left|\mathcal{O}_{i, \tau}^{\mu_{1} \ldots \mu_{n}}\right| p\right\rangle
\end{aligned}
$$


onde $c_{i, \mu_{1} \ldots \mu_{n}}^{\tau, \mu \nu}(q)$ representa a parte perturbativa do espalhamento, $\tau$ é o twist do operador e $i$ representa os diferentes operadores com o mesmo twist. Toda a informação não perturbativa está contida nos elementos de matriz.

Considerando agora os elementos de matriz dos operadores que possuem twist 2, substitui-se (2.28) por [26]:

$$
\left\langle p\left|\mathcal{O}_{\tau=2}^{\mu_{1} \ldots \mu_{n}}\right| p\right\rangle=\mathcal{A}_{2 k} \Pi^{\mu_{1} \ldots \mu_{2 k}}
$$

onde $\Pi^{\mu_{1} \ldots \mu_{2 k}}$ é um tensor sem traço e simétrico, que possui todas as contrações possíveis com um número arbitrário de $g_{\mu_{i} \mu_{j}}$ :

$$
\Pi^{\mu_{1} \ldots \mu_{2 k}}=\sum_{j=0}^{k}(-1)^{j} \frac{(2 k-j) !}{2^{j}(2 k) !} \underbrace{\{g \ldots g\}}_{j g^{\mu_{i} \mu_{j}}} \underbrace{\{p \ldots p\}}_{(2 k-2 j) p^{\mu_{i} s}}\left(p^{2}\right)^{j}
$$

sendo $\{g \ldots g\}\{p \ldots p\}$ uma abreviação para a soma simétrica de $\frac{(2 k) !}{2^{j} j !(2 k-2 j) !}$ produtos das distintas permutações dos índices de $g^{\prime} s$ e $p^{\prime} s$. Em (3.6) o termo $j=0$ reproduz o caso sem as TMC e $j>0$ produz as TMC.

A equação (3.4) pode ser reescrita como [27]:

$$
\begin{array}{r}
T^{\mu \nu}=\sum_{k=1}^{\infty}\left[-g^{\mu \nu} q_{\mu_{1}} q_{\mu_{2}} C_{1}^{2 k}+g_{\mu_{1}}^{\mu} g_{\mu_{2}}^{\nu} Q^{2} C_{2}^{2 k}-i \epsilon^{\mu \nu \lambda \sigma} g_{\lambda \mu_{1}} q_{\sigma} q_{\mu_{2}} C_{3}^{2 k}+\frac{q^{\mu} q^{\nu}}{Q^{2}} q_{\mu_{1}} q_{\mu_{2}} C_{4}^{2 k}\right. \\
\left.+\left(g_{\mu_{1}}^{\mu} q^{\nu} q_{\mu_{2}}+g_{\mu_{1}}^{\nu} q^{\mu} q_{\mu_{2}}\right) C_{5}^{2 k}\right] q_{\mu_{3}} \ldots q_{\mu_{2 k}} \frac{2^{2 k}}{\left(Q^{2}\right)^{2 k}} \mathcal{A}_{2 k} \Pi^{\mu_{1} \ldots \mu_{2 k}} .
\end{array}
$$

A amplitude Compton pode ser escrita também em termos de cinco estruturas tensoriais permitidas pela invariância de Lorentz, possuindo uma forma parecida com (3.2), explicitamente [27]:

$$
T^{\mu \nu}=-g^{\mu \nu} T_{1}+\frac{p^{\mu} p^{\nu}}{m_{p}^{2}} T_{2}-i \epsilon^{\mu \nu \lambda \sigma} \frac{p^{\lambda} q^{\sigma}}{m_{p}^{2}} T_{3}+\frac{q^{\mu} q^{\nu}}{m_{p}^{2}} T_{4}+\frac{p^{\mu} q^{\nu}+p^{\nu} q^{\mu}}{m_{p}^{2}} T_{5}
$$

onde iremos encontrar cada coeficiente de $T^{\mu \nu}$ e relacioná-lo com a expressão (3.2). 
Vamos derivar as correções em $\mu$ para a função de estrutura $F_{2}$; para as outras funções de estrutura veja o Apêndice A.

Primeiro é preciso encontrar $\frac{T_{2}}{m_{p}^{2}}$, que é o coeficiente de $p^{\mu} p^{\nu}$ em (3.8). Este terá contribuição do coeficiente do termo $C_{2}^{2 k}$ pela contração:

$$
\left(g_{\mu_{1}}^{\mu} g_{\mu_{2}}^{\nu} Q^{2} C_{2}^{2 k}\right)\left(p^{\mu_{1}} p^{\mu_{2}}\right)=p^{\mu} p^{\nu} Q^{2} C_{2}^{2 k}
$$

Assim, fixando $k \in \mathcal{N}$ e $j \in\{0, \ldots, k\}$, utilizando os termos de $\{g \ldots g\}\{p \ldots p\}$ que contenham $p^{\mu_{i}} p^{\mu_{l}}$, distribuindo os índices $\mu_{3}, \ldots, \mu_{2 k}$ pelos $j g^{\mu_{i} \mu_{l}}$ que se tem em (3.6) e pelos $(2 k-2 j-2) p^{\mu_{i}}$ (em $(3.6)$ tinha-se $(2 k-2 j) p^{\mu_{i}}$, para não criar produtos duplicados subtraiu-se os dois $p^{\mu_{i}}$ utilizados na identidade (3.9)), e fazendo $k \rightarrow k-1$ na soma simétrica (3.6), tem-se:

$$
\begin{aligned}
& \sum_{k=1}^{\infty}\left(g_{\mu_{1}}^{\mu} g_{\mu_{2}}^{\nu} Q^{2} C_{2}^{2 k}\right) q_{\mu_{3}} \ldots q_{\mu_{2 k}} \frac{2^{2 k}}{\left(Q^{2}\right)^{2 k}} \mathcal{A}_{2 k} \Pi^{\mu_{1} \ldots \mu_{2 k}}= \\
& =\quad p^{\mu} p^{\nu} \sum_{k=1}^{\infty} \frac{2^{2 k}}{\left(Q^{2}\right)^{2 k}} \sum_{j=0}^{k-1}(-1)^{j} \frac{(2 k-j) !}{2^{j}(2 k) !} \frac{(2 k-2) !}{2^{j} j !(2 k-2 j-2) !}\left(p^{2} q^{2}\right)^{j}(p \cdot q)^{2 k-2 j-2} \\
& \times \quad Q^{2} C_{2}^{2 k} \mathcal{A}_{2 k}+\text { termos sem } p^{\mu_{i}} p^{\mu_{l}} .
\end{aligned}
$$

Então:

$$
\begin{aligned}
\frac{T_{2}\left(x, Q^{2}\right)}{m_{p}^{2}} & =Q^{2} \sum_{k=1}^{\infty} \frac{2^{2 k}}{\left(Q^{2}\right)^{2 k}} \sum_{j=0}^{k-1}(-1)^{j} \frac{(2 k-j) !}{2^{j}(2 k) !} \frac{(2 k-2) !}{2^{j} j !(2 k-2 j-2) !} \\
& \times\left(p^{2} q^{2}\right)^{j}(p \cdot q)^{2 k-2 j-2} C_{2}^{2 k} \mathcal{A}_{2 k} .
\end{aligned}
$$

Pode-se verificar a consistência da equação (3.11), como exemplo, fazendo a soma até $k=2$ :

$$
\frac{\left.T_{2}\left(x, Q^{2}\right)\right|_{k_{\max }=2}}{m_{p}^{2}}=\frac{4}{Q^{2}}\left[C_{2}^{(2)} A_{2}+\frac{4}{Q^{2}} C_{2}^{(4)} A_{4}\left((p \cdot q)^{2}-p^{2} q^{2}\right)\right]
$$


De (3.6):

$$
\begin{aligned}
\Pi^{\mu_{1} \mu_{2}}= & p^{\mu_{1}} p^{\mu_{2}}-\frac{p^{2}}{4} g^{\mu_{1} \mu_{2}}, \\
\Pi^{\mu_{1} \mu_{2} \mu_{3} \mu_{4}}= & p^{\mu_{1}} p^{\mu_{2}} p^{\mu_{3}} p^{\mu_{4}}-\frac{p^{2}}{4}\left(g^{\mu_{1} \mu_{2}} p^{\mu_{3}} p^{\mu_{4}}+g^{\mu_{3} \mu_{4}} p^{\mu_{1}} p^{\mu_{2}}\right) \\
& +\frac{p^{4}}{8} g^{\mu_{1} \mu_{2}} g^{\mu_{3} \mu_{4}} .
\end{aligned}
$$

Utilizando o termo $C_{2}^{2 k}$ e seu coeficiente, da equação (3.7), fazendo a soma até $k=2$ e inserindo (3.13), chegamos em:

$$
\begin{aligned}
& \left(g_{\mu_{1}}^{\mu} g_{\mu_{1}}^{\nu} Q^{2} C_{2}^{(2)}\right) \frac{2^{2}}{\left(Q^{2}\right)^{2}} A_{2} \Pi^{\mu_{1} \mu_{2}}+\left(g_{\mu_{1}}^{\mu} g_{\mu_{1}}^{\nu} Q^{2} C_{2}^{(4)}\right) q_{\mu_{3}} q_{\mu_{4}} \frac{2^{4}}{\left(Q^{2}\right)^{4}} A_{4} \Pi^{\mu_{1} \mu_{2} \mu_{3} \mu_{4}}= \\
= & \left(p^{\mu} p^{\nu} \frac{2^{2}}{Q^{2}}-g^{\mu \nu} \frac{p^{2}}{Q^{2}}\right) C_{2}^{(2)} A_{2}+\left[p^{\mu} p^{\nu}(p \cdot q)^{2} \frac{2^{4}}{\left(Q^{2}\right)^{2}}-p^{2} \frac{2^{2}}{\left(Q^{2}\right)^{2}}\left(g^{\mu \nu}(p \cdot q)^{2}\right.\right. \\
& \left.\left.+p^{\mu} p^{\nu} q^{2}\right)+g^{\mu \nu} p^{4} q^{2} \frac{2}{\left(Q^{2}\right)^{2}}\right] C_{2}^{(4)} A_{4} \\
= & p^{\mu} p^{\nu}\left\{\frac{4}{Q^{2}}\left[C_{2}^{(2)} A_{2}+\frac{4}{Q^{2}} C_{2}^{(4)} A_{4}\left((p \cdot q)^{2}-p^{2} q^{2}\right)\right]\right\} \\
& +g^{\mu \nu}\left\{\frac{p^{2}}{Q^{2}}\left[-C_{2}^{(2)} A_{2}+\frac{2}{Q^{2}} C_{2}^{(4)} A_{4}\left(-2(p \cdot q)^{2}+p^{2} q^{2}\right)\right]\right\}
\end{aligned}
$$

considerando somente os termos proporcionais a $p^{\mu} p^{\nu}$, primeira parte de (3.14), obtemos (3.12), verificando sua consistência.

Voltando em (3.11) e substituindo $-q^{2}=Q^{2}, x=\frac{Q^{2}}{2 p \cdot q}$ e $p^{2}=m_{p}^{2}$ :

$$
\begin{aligned}
\frac{T_{2}\left(x, Q^{2}\right)}{m_{p}^{2}} & =Q^{2} \sum_{k=1}^{\infty} \frac{2^{2 k}}{\left(Q^{2}\right)^{2 k}} \sum_{j=0}^{k-1}(-1)^{j} \frac{(2 k-j) !}{2^{j}(2 k) !} \frac{(2 k-2) !}{2^{j} j !(2 k-2 j-2) !} \\
& \times\left(m_{p}^{2}\right)^{j}\left(-Q^{2}\right)^{j}\left(\frac{Q^{2}}{2 x}\right)^{2 k-2 j-2} C_{2}^{2 k} \mathcal{A}_{2 k}
\end{aligned}
$$




$$
\begin{aligned}
& =\frac{2}{Q^{2}} \sum_{k=1}^{\infty} \sum_{j=0}^{k-1} \frac{(2 k-j) !}{j !(2 k-2 j) !} \frac{(2 k-2 j)(2 k-2 j-1)}{k(2 k-1)} \\
& \times\left(\frac{m_{p}^{2}}{Q^{2}}\right)^{j} \frac{C_{2}^{2 k} \mathcal{A}_{2 k}}{x^{2 k-2 j-2}} .
\end{aligned}
$$

Utilizando o binômio $\left(\begin{array}{l}n \\ k\end{array}\right)=\frac{n !}{k !(n-k) !}$ e fazendo a troca $k=l+j+1$ em $(3.15)$, tem-se:

$$
\begin{aligned}
T_{2}\left(x, Q^{2}\right) & =4 \sum_{l=0}^{\infty} \sum_{j=0}^{\infty}\left(\begin{array}{c}
2 l+j+2 \\
j
\end{array}\right) \mu^{j+1} \frac{(2 l+2)(2 l+1)}{(2 l+2 j+2)(2 l+2 j+1)} \\
& \times \frac{C_{2}^{2 l+2 j+2} \mathcal{A}_{2 l+2 j+2}}{x^{2 l}} .
\end{aligned}
$$

Lembrando que $x=\frac{1}{\omega}$ e usando as equações (2.18), (2.19), (2.35) com $i=2$, e as equações (3.3) e (3.16), encontra-se o momento de Cornwall-Norton:

$$
\begin{aligned}
M_{2}^{(n)}\left(Q^{2}\right) & =\int_{0}^{1} x^{n-2} F_{2}^{T M C}\left(x, Q^{2}\right) d x=\frac{1}{2} \frac{1}{2 \pi i} \oint_{C} \frac{1}{\omega^{n}} \frac{\omega}{2 \mu} T_{2}\left(1 / \omega, Q^{2}\right) d \omega \\
& =\sum_{l=0}^{\infty} \sum_{j=0}^{\infty}\left(\begin{array}{c}
2 l+j+2 \\
j
\end{array}\right) \mu^{j} \frac{(2 l+2)(2 l+1) C_{2}^{2 l+2 j+2} \mathcal{A}_{2 l+2 j+2}}{(2 l+2 j+2)(2 l+2 j+1)} \\
& \times\left(\frac{1}{2 \pi i} \oint_{C} \omega^{2 l-n+1} d \omega\right) \\
& =\sum_{l=0}^{\infty} \sum_{j=0}^{\infty}\left(\begin{array}{c}
2 l+j+2 \\
j
\end{array}\right) \mu^{j} \frac{(2 l+2)(2 l+1) C_{2}^{2 l+2 j+2} \mathcal{A}_{2 l+2 j+2}}{(2 l+2 j+2)(2 l+2 j+1)} \delta_{2 l, n-2} \\
M_{2}^{(n)}\left(Q^{2}\right) & =\sum_{j=0}^{\infty}\left(\begin{array}{c}
n+j \\
j
\end{array}\right) \mu^{j} \frac{n(n-1)}{(n+2 j)(n+2 j-1)} C_{2}^{n+2 j} \mathcal{A}_{n+2 j} .
\end{aligned}
$$

Note que para $j=0$ recupera-se a equação (2.34), ou seja, $M_{2}^{(n)}\left(Q^{2}\right)$ sem TMC. O sobrescrito $T M C$ em $F_{2}\left(x, Q^{2}\right)$ enfatiza a incorporação da correção da massa na função de estrutura.

A derivação detalhada para os outros Momentos de Cornwall-Norton encontra-se 
no Apêndice A e são dados por:

$$
\begin{aligned}
& M_{1}^{(n)}\left(Q^{2}\right)=\sum_{j=0}^{\infty}\left(\begin{array}{c}
n+j \\
j
\end{array}\right) \mu^{j}\left[\frac{C_{1}^{n+2 j}}{2}+\frac{j C_{2}^{n+2 j}}{(n+2 j)(n+2 j-1)}\right] \mathcal{A}_{n+2 j} \\
& M_{L}^{(n)}\left(Q^{2}\right)=\sum_{j=0}^{\infty}\left(\begin{array}{c}
n+j \\
j
\end{array}\right) \mu^{j}\left[C_{L}^{n+2 j}+\frac{4 j C_{2}^{n+2 j}}{(n+2 j)(n+2 j-1)}\right] \mathcal{A}_{n+2 j} ; \\
& M_{3}^{(n)}\left(Q^{2}\right)=\sum_{j=0}^{\infty}\left(\begin{array}{c}
n+j \\
j
\end{array}\right) \mu^{j} \frac{n}{n+2 j} C_{3}^{n+2 j} \mathcal{A}_{n+2 j} \\
& M_{4}^{(n)}\left(Q^{2}\right)=\sum_{j=0}^{\infty}\left(\begin{array}{c}
n+j \\
j
\end{array}\right) \mu^{j}\left[\frac{j(j-1)}{(n+2 j)(n+2 j-1)} C_{2}^{n+2 j}+\frac{1}{4} C_{4}^{n+2 j}\right. \\
& \left.-\frac{j}{(n+2 j)(n+2 j-1)} C_{5}^{n+2 j}\right] \mathcal{A}_{n+2 j} \text {; } \\
& M_{5}^{(n)}\left(Q^{2}\right)=\sum_{j=0}^{\infty}\left(\begin{array}{c}
n+j \\
j
\end{array}\right) \mu^{j} \frac{n}{n+2 j}\left[-\frac{j C_{2}^{n+2 j}}{n+2 j-1}+\frac{C_{5}^{n+2 j}}{2}\right] \mathcal{A}_{n+2 j},
\end{aligned}
$$

onde $C_{L}^{n+2 j}=C_{2}^{n+2 j}-C_{1}^{n+2 j}$. A expressão para $M_{1}^{(n)}$ é a mesma que a encontrada em [76] desde que as diferenças entre as definições dos operadores correspondentes sejam levadas em conta.

\subsubsection{Funções de estrutura com as TMC}

Para encontrarmos a dependência em $x$ das funções de estrutura com TMC, precisamos utilizar a transformada inversa de Mellin [77]:

$$
F_{i}^{T M C}\left(x, Q^{2}\right)= \begin{cases}\frac{1}{2 \pi i} \int_{-i \infty}^{i \infty} d n x^{-n} M_{i}^{(n)}\left(Q^{2}\right), & \text { se } i=1,3,4,5 \\ \frac{1}{2 \pi i} \int_{-i \infty}^{i \infty} d n x^{-n+1} M_{i}^{(n)}\left(Q^{2}\right), & \text { se } i=2, L\end{cases}
$$

O elemento de matriz $\mathcal{A}_{n+2 j}$ é definido como o momento de uma distribuição de pártons 
universal $f_{i}(x)$, por isso pode-se escrever o produto $C_{i}^{n+2 j} \mathcal{A}_{n+2 j}$ como [78]:

$$
C_{i}^{n+2 j} \mathcal{A}_{n+2 j}=\int_{0}^{1} d x x^{n+2 j-1} f_{i}(x)
$$

onde $f_{i}(x)$ está relacionada com as funções de estrutura no limite sem massa $F_{i}^{(0)} \equiv$ $\lim _{\mu \rightarrow 0} F_{i}^{T M C}[78]:$

$$
\left\{F_{1}^{(0)}, F_{2}^{(0)}, F_{L}^{(0)}, F_{3}^{(0)}, F_{4}^{(0)}, F_{5}^{(0)}\right\}=\left\{\frac{f_{1}}{2}, x f_{2}, x\left(f_{2}-f_{1}\right), f_{3}, \frac{f_{4}}{4}, \frac{f_{5}}{2}\right\} .
$$

Para o caso da equação (3.17):

$$
\frac{C_{2}^{n+2 j} \mathcal{A}_{n+2 j}}{(n+2 j)(n+2 j-1)}=\int_{0}^{1} d y y^{n+2 j-2} g_{2}(y),
$$

onde

$$
g_{i}(y)=\int_{y}^{1} d u h_{i}(u) \quad \text { e } \quad h_{i}(u)=\int_{u}^{1} d x \frac{f_{i}(x)}{x} .
$$

Então, utilizando-se a transformada inversa de Mellin, (3.19) para $i=2$, na equação (3.17) com (3.22), tem-se:

$$
\begin{aligned}
F_{2}^{T M C}\left(x, Q^{2}\right) & =\frac{1}{2 \pi i} \int_{0}^{1} d y \int_{-i \infty}^{+i \infty} d n x^{-n+1} \sum_{j=0}^{\infty}\left(\begin{array}{c}
n+j \\
j
\end{array}\right) n(n-1) \mu^{j} y^{n+2 j-2} g_{2}(y) \\
& =\frac{1}{2 \pi i} \int_{0}^{1} d y \int_{-i \infty}^{+i \infty} d n x^{-n+1} y^{n} \sum_{j=0}^{\infty}\left(\begin{array}{c}
n+j \\
j
\end{array}\right)\left(\mu y^{2}\right)^{j} \\
& \times n(n-1) \frac{g_{2}(y)}{y^{2}}
\end{aligned}
$$

Pelo teorema binomial generalizado, pode-se eliminar a soma em $j$ :

$$
\sum_{j=0}^{\infty}\left(\begin{array}{c}
n+j \\
j
\end{array}\right)\left(\mu y^{2}\right)^{j}=\frac{1}{\left(1-\mu y^{2}\right)^{n+1}}
$$


Com $(3.25)$ e $n(n-1) x^{-n+1}=x^{2} \frac{\partial^{2}}{\partial x^{2}} x^{1-n},(3.24)$ simplifica para:

$$
\begin{aligned}
F_{2}^{T M C}\left(x, Q^{2}\right) & =x^{2} \frac{\partial^{2}}{\partial x^{2}}\left[\frac{1}{2 \pi i} \int_{0}^{1} d y \int_{-i \infty}^{+i \infty} d n x^{1-n} y^{n} \frac{g_{2}(y)}{y^{2}\left(1-\mu y^{2}\right)^{n+1}}\right] \\
& =x^{2} \frac{\partial^{2}}{\partial x^{2}}\left\{\int_{0}^{1} d y \frac{x g_{2}(y)}{y^{2}\left(1-\mu y^{2}\right)}\left[\frac{1}{2 \pi i} \int_{-i \infty}^{+i \infty} d n\left(\frac{y}{x\left(1-\mu y^{2}\right)}\right)^{n}\right]\right\} \\
& =x^{2} \frac{\partial^{2}}{\partial x^{2}}\left\{\int_{0}^{1} d y \frac{x g_{2}(y)}{y^{2}\left(1-\mu y^{2}\right)} \delta\left[\ln \left(\frac{y}{x\left(1-\mu y^{2}\right)}\right)\right]\right\},
\end{aligned}
$$

onde foi usada a propriedade da função $\delta$ :

$$
\frac{1}{2 \pi i} \int_{-i \infty}^{+i \infty} d n z^{n}=\frac{1}{2 \pi} \int_{-\infty}^{\infty} d n e^{i n \ln (z)}=\delta[\ln (z)]
$$

Para resolver a integral, emprega-se a relação:

$$
\delta[f(z)]=\sum_{i} \frac{\delta\left(z-z_{i}\right)}{\left|f^{\prime}\left(z_{i}\right)\right|}
$$

sendo $z_{i}$ as raízes de $\delta[f(z)]$. Com $f(y)=\ln \left(\frac{y}{x\left(1-\mu y^{2}\right)}\right)$ e $f^{\prime}(y)=\frac{1+\mu y^{2}}{y\left(1-\mu y^{2}\right)}$, a única raíz para $y \in[0,1]$ é $y=\xi=\frac{2 x}{1+\rho}$. Assim, a variável de Nachtmann, (3.1), aparece naturalmente quando as TMC são incorporadas no cálculo das funções de estrutura. A equação (3.26) é, finalmente, reescrita como:

$$
\begin{aligned}
& F_{2}^{T M C}\left(x, Q^{2}\right)=x^{2} \frac{\partial^{2}}{\partial x^{2}}\left[\int_{0}^{1} d y \frac{x g_{2}(y)}{y^{2}\left(1-\mu y^{2}\right)} \delta(y-\xi) \frac{\xi\left(1-\mu \xi^{2}\right)}{1+\mu \xi^{2}}\right] \\
& F_{2}^{T M C}\left(x, Q^{2}\right)=x^{2} \frac{\partial^{2}}{\partial x^{2}}\left[\frac{x g_{2}(\xi)}{\xi\left(1+\mu \xi^{2}\right)}\right] .
\end{aligned}
$$

Na Figura (3.2) é mostrada a influência da inclusão das TMC para a função de estrutura, equação (3.29), onde foi utilizada a PDF dada pela MSTW 2008 [16]. Analisando a Figura (3.2) nota-se que a maior diferença é na região de baixo $Q^{2}$ e grande 
$x$. Quando se aumenta $Q^{2}$ a função com TMC aproxima-se da distribuição sem TMC. Claramente percebe-se que a função de estrutura com TMC não é nula para $x \rightarrow 1$, implicando em uma violação da conservação de energia e momento (esta violação será estudada mais profundamente na Seção 3.3).

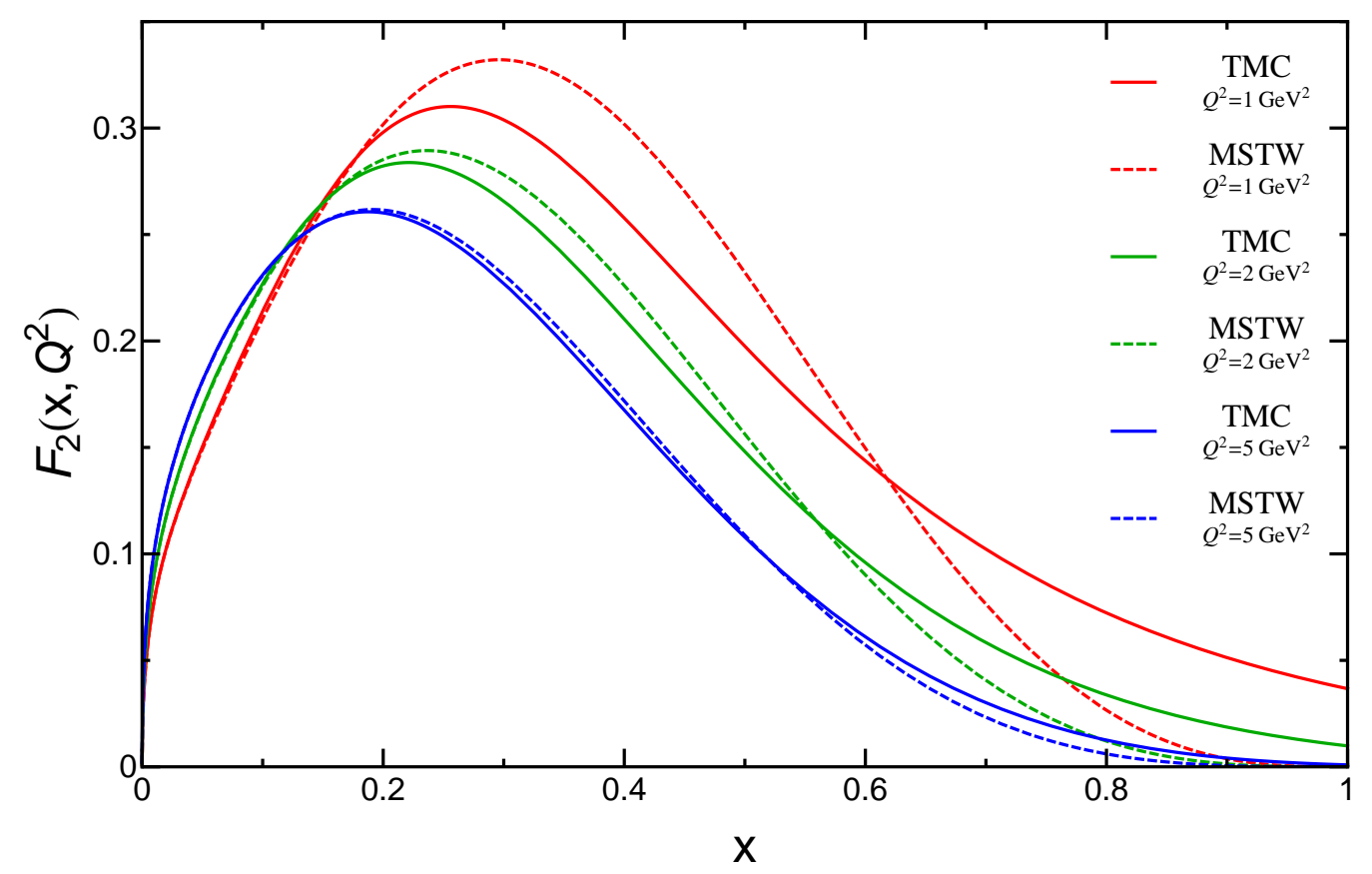

Figura 3.2: $F_{2}$ com (linhas contínuas) e sem TMC (linhas tracejadas) para vários $Q^{2}$, sendo a PDF dada pela MSTW 2008 [16].

A Figura (3.3) mostra uma comparação entre a função de estrutura $F_{2}$ e os dados do experimento NuTeV [79] com (linha contínua) e sem (linha pontilhada) TMC, onde foi utilizada a PDF CTEQ6HQ [80]. Verifica-se que com a inclusão das TMC há uma melhora na função de estrutura na região de alto $x$, em comparação com os dados experimentais.

Seguindo os passos feitos para encontrar a equação (3.29), tem-se as outras funções de estrutura (a dedução detalhada encontra-se no Apêndice A):

$$
F_{1}^{T M C}\left(x, Q^{2}\right)=\frac{f_{1}(\xi)}{2\left(1+\mu \xi^{2}\right)}-\mu x^{2} \frac{\partial}{\partial x}\left[\frac{g_{2}(\xi)}{1+\mu \xi^{2}}\right]
$$




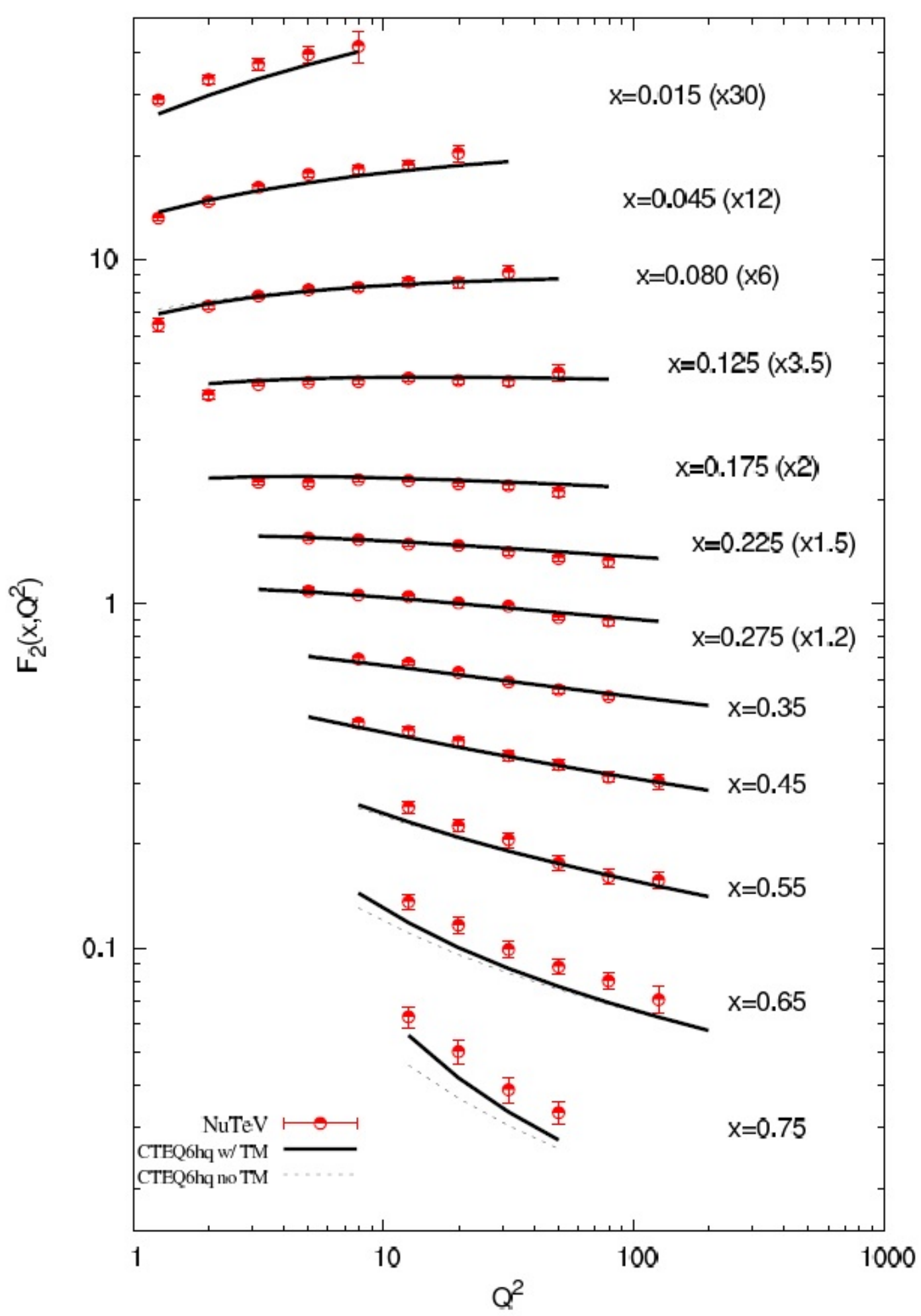

Figura 3.3: Comparação da função de estrutura $F_{2}$ com (linhas contínuas) e sem TMC (linhas tracejadas), junto com dados do NuTeV [79], sendo a PDF dada pela CTEQ6HQ [80]. Extraído de [27]. 


$$
\begin{aligned}
F_{L}^{T M C}\left(x, Q^{2}\right)= & -\frac{x f_{1}(\xi)}{1+\mu \xi^{2}}+2 \mu x^{3} \frac{\partial}{\partial x}\left[\frac{g_{2}(\xi)}{1+\mu \xi^{2}}\right] \\
& +\left(1+4 \mu x^{2}\right) x^{2} \frac{\partial^{2}}{\partial x^{2}}\left[\frac{x g_{2}(\xi)}{\xi\left(1+\mu \xi^{2}\right)}\right] ; \\
F_{3}^{T M C}\left(x, Q^{2}\right)= & -x \frac{\partial}{\partial x}\left[\frac{h_{3}(\xi)}{1+\mu \xi^{2}}\right] ; \\
F_{4}^{T M C}\left(x, Q^{2}\right)= & \mu^{2} x^{3} \frac{\partial^{2}}{\partial x^{2}}\left[\frac{\xi^{2} g_{2}(\xi)}{1-\mu^{2} \xi^{4}}\right]+\frac{f_{4}(\xi)}{4\left(1+\mu \xi^{2}\right)} \\
& +\mu x^{2} \frac{\partial}{\partial x}\left[\frac{g_{5}(\xi)}{1+\mu \xi^{2}}\right] ; \\
F_{5}^{T M C}\left(x, Q^{2}\right)= & -\mu x^{2} \frac{\partial^{2}}{\partial x^{2}}\left[\frac{\xi g_{2}(\xi)}{1-\mu^{2} \xi^{4}}\right]-\frac{x}{2} \frac{\partial}{\partial x}\left[\frac{h_{5}(\xi)}{1+\mu \xi^{2}}\right] .
\end{aligned}
$$

Note que a expressão para a função de estrutura $F_{4}$ obtida acima, equação (3.30d), contêm $g_{5}(\xi)$ ao invés de $\xi h_{5}(\xi)$ encontrada pelos autores de [75].

Como havia sido mencionado na Seção 1.3, a implementação das TMC nas funções de estrutura leva à violação da relação de Callan-Gross, equação (1.33), pois a função de estrutura longitudinal $F_{L}^{T M C}\left(x, Q^{2}\right)$ é dada por $(3.30 \mathrm{~b})$. Ou seja, $F_{L}^{T M C}\left(x, Q^{2}\right)=$ $\left(1+4 \mu x^{2}\right) F_{2}^{T M C}\left(x, Q^{2}\right)-2 x F_{1}^{T M C}\left(x, Q^{2}\right)$, que em geral não é nulo.

\subsection{Momento de Nachtmann}

Os momentos de Nachtmann $\left(\Omega_{i}^{(n)}\left(Q^{2}\right)\right)$, em princípio, protegem as funções de estrutura das TMC, no sentido de, por exemplo, $\Omega_{2}^{(n)}=A_{n}$, diferente de (3.17) que é uma série infinita em $\frac{m_{p}^{2}}{Q^{2}}$. Nachtmann [25] mostrou que se pode rearranjar a OPE de modo que somente operadores com determinado twist aparecem para dada ordem em $\frac{1}{Q^{2}}$ [81]. Sua construção é feita a partir de operadores com spin definido [27]. Então dos diversos operadores de twist 2, somente os operadores com spin $n$ contribuem para o momento de Nachtman $n-2$ [27], diferente do momento de Cornwall-Norton que engloba os operadores com spin diferentes [27,82]. O momento de Nachtmann se reduz 
ao momento de Cornwall-Norton $[27,82]$ quando $Q^{2} \rightarrow \infty$.

A derivação consiste em utilizar a amplitude do espalhamento Compton virtual (2.24) com a OPE (2.25), calcular o valor esperado, fazer uma transformada de Fourier, realizar as contrações e utilizar as relações de recorrência dos polinômios de Gegenbauer. Um pouco de álgebra, mostra que [27, 25, 82, 83, 84]:

$$
\begin{aligned}
\Omega_{1}^{(n)}\left(Q^{2}\right)= & -\int_{0}^{1} d x \frac{\xi^{n+1}}{x^{2}}\left[2 F_{1}^{T M C}\left(x, Q^{2}\right)-\frac{\rho^{2}}{3 x} F_{2}^{T M C}\left(x, Q^{2}\right)\right] \\
\Omega_{2}^{(n)}\left(Q^{2}\right)= & \int_{0}^{1} d x \frac{\xi^{n+1}}{x^{3}}\left[\frac{3+3(n+1) \rho+n(n+2) \rho^{2}}{(n+2)(n+3)}\right] F_{2}^{T M C}\left(x, Q^{2}\right) ; \\
\Omega_{L}^{(n)}\left(Q^{2}\right)= & \int_{0}^{1} d x \frac{\xi^{n+1}}{x^{3}}\left\{F_{L}^{T M C}\left(x, Q^{2}\right)\right. \\
& \left.+\left(\rho^{2}-1\right)\left[\frac{(n+1) \frac{\xi}{x}-2(n+2)}{(n+2)(n+3)}\right] F_{2}^{T M C}\left(x, Q^{2}\right)\right\} \\
\Omega_{3}^{(n)}\left(Q^{2}\right)= & \int_{0}^{1} d x \frac{\xi^{n+1}}{x^{2}}\left[\frac{1+(n+1) \rho}{(n+2)}\right] F_{3}^{T M C}\left(x, Q^{2}\right) .
\end{aligned}
$$

O momento de Nachtmann se relaciona com o momento de Cornwall-Norton através de uma expansão em potências de $\frac{1}{Q^{2}}$. Expandindo $(3.31 b)$ até $\mathcal{O}\left(\frac{1}{Q^{6}}\right)$, tem-se [85]:

$$
\begin{aligned}
\Omega_{2}^{(n)}\left(Q^{2}\right)= & M_{2}^{(n)}\left(Q^{2}\right)-\frac{n(n-1)}{n+2} \mu M_{2}^{(n+2)}\left(Q^{2}\right)+\frac{n\left(n^{2}-1\right)}{2(n+3)} \mu^{2} M_{2}^{(n+4)}\left(Q^{2}\right) \\
& -\frac{n\left(n^{2}-1\right)}{6} \mu^{3} M_{2}^{(n+6)}\left(Q^{2}\right)+\mathcal{O}\left(\frac{1}{Q^{8}}\right)
\end{aligned}
$$

onde se percebe a mistura entre momentos de Cornwall-Norton de primeira e mais alta ordem para um dado momento de Nachtmann. Verifica-se, trivialmente, que no limite sem massa $(\mu=0)$ o momento de Nachtmann se resume ao momento de Cornwall-Norton.

Na Figura (3.4) temos uma comparação entre o momento de Nachtmann com $n=2, \Omega_{2}^{(n=2)}\left(Q^{2}\right)$ equação (3.31b), usando a função de estrutura com TMC (3.29), e 
o momento de Cornwall-Norton com $n=2, M_{2}^{(n=2)}\left(Q^{2}\right)$, sem TMC. A distribuição de pártons usada é a CTEQ6HQ [80].

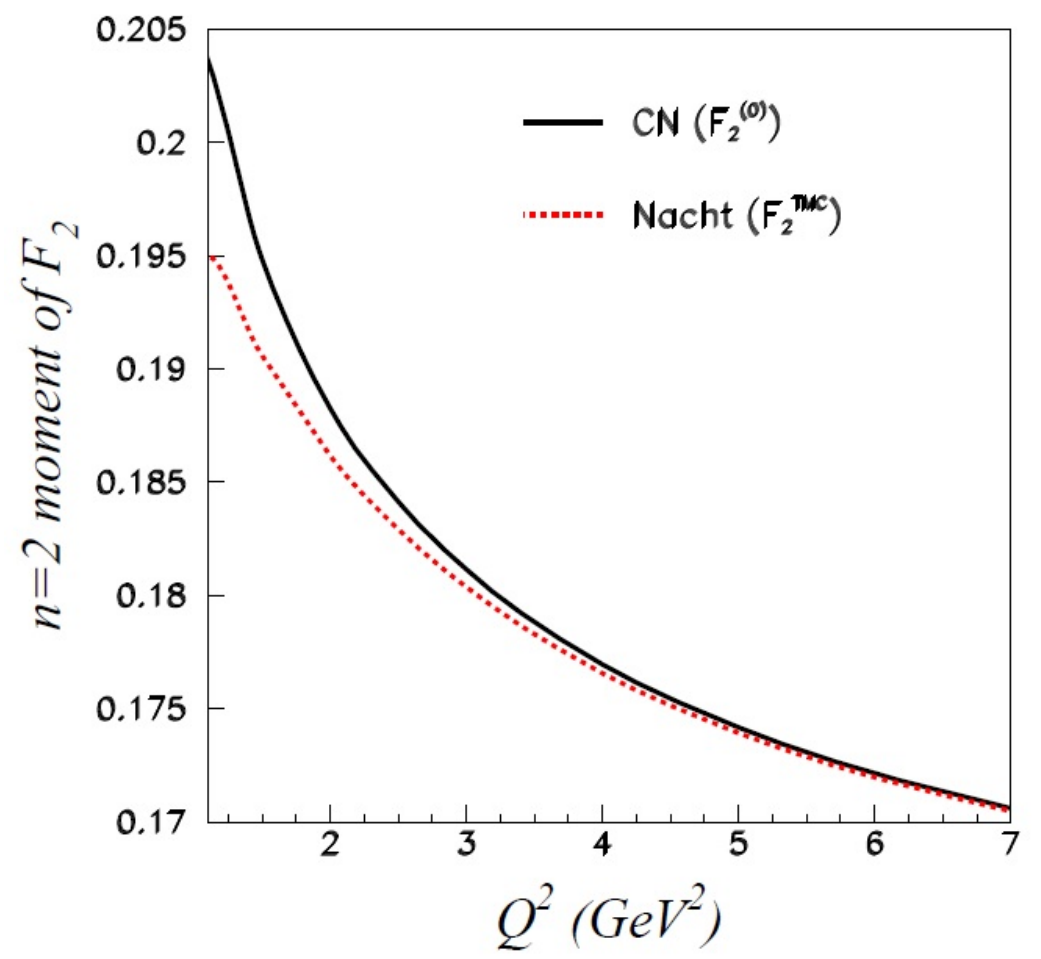

Figura 3.4: Comparação do momento de Cornwall-Norton (linha contínua) com $n=2$ e sem TMC, com o momento de Nachtmann (linha tracejada) (3.31b) com $n=2$, usando a TMC (3.29) e com a mesma distribuição utilizada no momento de Cornwall-Norton. Extraído de [27].

Vê-se que para grande $Q^{2}$ os momentos de Nachtmann e de Cornwall-Norton coincidem, mas para $Q^{2}$ suficientemente pequeno percebe-se um claro desvio entre os dois, que é causado pelo comportamento da função de estrutura com TMC no limite $x \rightarrow 1$ [27]. Portanto, seria incorreto fazer uma análise das funções de estrutura na região de pequeno $Q^{2}$ usando os momentos de Cornwall-Norton sem as TMC, pois, neste caso seria incorreto usar $\Omega_{2}^{(n)}=A_{n}$.

\subsection{O problema com o scaling $\xi$}

A abordagem usada por Georgi e Politzer para tratar as TMC apresenta alguns 
problemas, que foram percebidos já nos primórdios da QCD [29, 30], na região próxima ao limite elástico $(x \rightarrow 1)$. Explicitamente, as funções expressas em termos de $\xi$ no intervalo $0 \leq \xi \leq 1$ se estendem até regiões não físicas, já que o limite elástico é:

$$
\xi_{0} \equiv \xi(x=1)=\frac{2}{1+\sqrt{1+4 \mu}}<1 \quad \text { para } \quad \mu \neq 0
$$

Assim entre $\xi_{0} \leq \xi \leq 1$ existe uma violação da conservação de energia e momento para qualquer $Q^{2}$ finito, além de fazer com que as funções de estrutura sejam não nulas em $x=1$ (em desacordo com o fato de que elas devem ser nulas no limite elástico [86]).

Uma possível resolução foi proposta por De Rujula et al. em 1977 [87], onde foi argumentado que no estudo das TMC é preciso considerar também os operadores com twists maiores. Em geral, pode-se escrever o momento de uma função de estrutura como [87]:

$$
M^{(n)}\left(Q^{2}\right)=\int_{0}^{1} d \xi \xi^{n} F\left(\xi, Q^{2}\right)=A_{n}\left(Q^{2}\right)+\sum_{i=1}^{\infty}\left(n \frac{m_{p}^{2}}{Q^{2}}\right)^{i} B_{n, i}\left(Q^{2}\right)
$$

onde o $i$-ésimo termo da soma é a contribuição dos operadores com twist $2 i+2$. Os autores de [87] notaram que se a função de estrutura se comporta como $(1-\xi)^{a}$ quando $\xi \rightarrow 1$, com $3 \leq a \leq 4$, a função $\xi^{n}(1-\xi)^{a}$ em (3.34) terá um máximo em $\xi_{n}=\frac{n}{n+a} \sim 1-\frac{a}{n}$ para $n$ grande. Assim, o n-ésimo momento, $M^{(n)}\left(Q^{2}\right)$, é sensível ao comportamento da função de estrutura para $\xi \simeq \xi_{n}$. Na região do limite elástico $(x=1)$ :

$$
\xi \simeq \xi_{\max }\left(Q^{2}\right)=1-\frac{m_{p}^{2}}{Q^{2}}+\mathcal{O}\left(\frac{m_{p}^{4}}{Q^{4}}\right)
$$

Para $n$ grande, $\xi_{n} \simeq \xi_{\text {max }}\left(Q^{2}\right)$ ou $n \approx a \frac{Q^{2}}{m_{p}^{2}}$, mas isto implica que twists maiores presentes na relação (3.34) necessitam ser incluídos. Assim, existe uma falta de uniformidade nos limites $n \rightarrow \infty$ (ou $\xi \rightarrow 1$ ) e $Q^{2} \rightarrow \infty$ que faz a abordagem insustentável para pequenos $W$ (massa invariante), pois aparecem efeitos de twists maiores $\propto n \frac{m_{p}^{2}}{Q^{2}}$ para 
o n-ésimo momento da função de estrutura. Porém o experimento realizado no JLab em 2000 [88] mostrou, através de uma razão integral entre a função de estrutura $F_{2}$ obtida de dados da ressonância do hidrogênio com outras $F_{2}$ obtidas por diferentes grupos, que o efeito de twists maiores são pequenos para a função de estrutura $F_{2}$ do próton em $Q^{2}$ pequeno $\left(\sim 0.5-1 \mathrm{GeV}^{2}\right)$. Esta questão ainda esta em aberto, já que nenhum trabalho mostrou, de fato, que a consideração de twists maiores resolvem o problema.

Tung e colaboradores [30] tentaram resolver este problema fenomenologicamente, utilizando um ansatz para juntar suavemente os momentos na região de QCD perturbativa (grande $Q^{2}$ ) com o comportamento correto no limite $n \rightarrow \infty$. Como os próprios autores notaram, esta prescrição não é única e somente coincide com a expansão da OPE no limite $n \rightarrow \infty$.

Ellis, Furmanski e Petronzio [89], evitaram a inversão dos momentos implementando as TMC diretamente no espaço dos momentos com o modelo de pártons [75, 90, 91]. Uma abordagem parecida foi utilizada por D’Alesio, Leader e Murgia [92], onde foi considerado que os pártons estão na sua camada de massa $\left(k^{2}=0\right)$ e possuem momento transverso, reproduzindo os resultados da OPE [93]. Mas esta abordagem leva a uma dependência na prescrição $[90,91]$ ou não se estende para todas as ordens em $\frac{1}{Q^{2}}$ [89], e ainda se tem o problema da inclusão da região não física $\xi_{0} \leq \xi \leq 1$.

Steffens e Melnitchouk [85] propuseram uma modificação na definição do momento da distribuição de párton, equação (3.20), tal que a integração é realizada somente na região fisicamente permitida:

$$
C_{i}^{n+2 j} \mathcal{A}_{n+2 j}=\int_{0}^{x_{0}} d x x^{n+2 j-1} f_{i}(x)
$$

onde $x_{0}$ é o valor máximo, fisicamente permitido, que a distribuição $f_{i}(x)$ pode assumir. Os autores obtiveram $x_{0}=\xi_{0}$, equação (3.33). Assim as funções de estrutura com TMC recebem contribuições somente de regiões fisicamente permitidas, porém o momento da distribuição de párton passa a depender de qual alvo está sendo usado e da virtualidade 
$\left(Q^{2}\right)$, já que $\xi_{0}$ depende de $\mu=\frac{m_{p}^{2}}{Q^{2}}$, fazendo-a não ser universal. Conclui-se que se for considerada somente a região fisicamente permitida, perde-se a interpretação partônica [93].

\subsubsection{Consistência na TMC com o scaling $\xi$}

Para que as funções de estrutura com TMC, equações (3.29) e (3.30), sejam consistentes é necessário obter os momentos das funções de estrutura (3.17) e (3.18) utilizando estas funções de estrutura com TMC, (3.29) e (3.30), na definição do momento de Cornwall-Norton (2.35), ou seja, fazer o caminho inverso. Como ilustração é feito este procedimento para $F_{2}$. Fazendo a substituição de (3.29) em $(2.35)$, com $i=2$ :

$$
\begin{aligned}
M_{2}^{(n)}\left(Q^{2}\right) & =\int_{0}^{1} d x x^{n-2} F_{2}^{T M C}\left(x, Q^{2}\right)=\int_{0}^{1} d x x^{n} \frac{\partial^{2}}{\partial x^{2}}\left[\frac{x g_{2}(\xi)}{\xi\left(1+\mu \xi^{2}\right)}\right] \\
& =\left.\left\{x^{n} \frac{\partial}{\partial x}\left[\frac{x g_{2}(\xi)}{\xi\left(1+\mu \xi^{2}\right)}\right]\right\}\right|_{0} ^{1}-n \int_{0}^{1} d x x^{n-1} \frac{\partial}{\partial x}\left[\frac{x g_{2}(\xi)}{\xi\left(1+\mu \xi^{2}\right)}\right] \\
& =\left.\left\{x^{n} \frac{\partial}{\partial x}\left[\frac{x g_{2}(\xi)}{\xi\left(1+\mu \xi^{2}\right)}\right]\right\}\right|_{0} ^{1}-\left.\left[n x^{n-1} \frac{x g_{2}(\xi)}{\xi\left(1+\mu \xi^{2}\right)}\right]\right|_{0} ^{1} \\
& +n(n-1) \int_{0}^{1} d x x^{n-2} \frac{x g_{2}(\xi)}{\xi\left(1+\mu \xi^{2}\right)} .
\end{aligned}
$$

Fazendo a mudança de variável de $x$ para $\xi, x=\frac{\xi}{\left(1-\mu \xi^{2}\right)} \rightarrow \frac{d x}{d \xi}=\frac{1+\mu \xi^{2}}{\left(1-\mu \xi^{2}\right)^{2}}$ e $\frac{d \xi}{d x}=$ $\frac{\left(\mu \xi^{2}-1\right)^{2}}{\mu \xi^{2}\left(\mu \xi^{2}+1\right)}$, o limite superior passa a ser $\xi_{0}$, equação (3.33), e:

$$
\begin{aligned}
M_{2}^{(n)}\left(Q^{2}\right) & =\left.\left[\left(\frac{\xi}{1-\mu \xi^{2}}\right)^{n} \frac{\left(\mu \xi^{2}-1\right)^{2}}{\mu \xi^{2}\left(\mu \xi^{2}+1\right)} \frac{\partial}{\partial \xi}\left(\frac{g_{2}(\xi)}{1-\mu^{2} \xi^{4}}\right)\right]\right|_{0} ^{\xi_{0}} \\
& -\left.\left[n\left(\frac{\xi}{1-\mu \xi^{2}}\right)^{n} \frac{g_{2}(\xi)}{\xi\left(1+\mu \xi^{2}\right)}\right]\right|_{0} ^{\xi_{0}} \\
& +n(n-1) \int_{0}^{\xi_{0}} d \xi \frac{\xi^{n-2}}{\left(1-\mu \xi^{2}\right)^{n+1}} g_{2}(\xi) .
\end{aligned}
$$


Usando o teorema binomial generalizado, equação (3.25), chega-se a:

$$
\begin{aligned}
M_{2}^{(n)}\left(Q^{2}\right) & =\sum_{j=0}^{\infty}\left(\begin{array}{c}
n+j \\
j
\end{array}\right) \mu^{j}\left[4 \mu \xi_{0}^{n+2 j+1} \frac{1-\mu \xi_{0}^{2}}{\left(1+\mu \xi_{0}^{2}\right)^{3}} g_{2}\left(\xi_{0}\right)\right. \\
& +\left.\frac{\xi_{0}^{n+2 j-2}}{\mu} \frac{\left(1-\mu \xi_{0}^{2}\right)^{2}}{\left(1+\mu \xi_{0}^{2}\right)^{2}} \frac{\partial g_{2}(\xi)}{\partial \xi}\right|_{\xi=\xi_{0}}-n \xi_{0}^{n+2 j-1} \frac{1-\mu \xi_{0}^{2}}{1+\mu \xi_{0}^{2}} g_{2}\left(\xi_{0}\right) \\
& \left.+n(n-1) \int_{0}^{\xi_{0}} d \xi \xi^{n+2 j-2} g_{2}(\xi)\right]
\end{aligned}
$$

Da definição do momento da função de distribuição de pártons (3.20), pode-se escrever:

$$
\begin{aligned}
\frac{\mathcal{A}_{n+2 j} C_{2}^{n+2 j}}{(n+2 j)(n+2 j-1)} & =\int_{0}^{1} d \xi \xi^{n+2 j-2} g_{2}(\xi) \\
& =\int_{0}^{\xi_{0}} d \xi \xi^{n+2 j-2} g_{2}(\xi)+\int_{\xi_{0}}^{1} d \xi \xi^{n+2 j-2} g_{2}(\xi)
\end{aligned}
$$

pois, de (3.33), $\xi_{0}<1$ para qualquer $Q^{2}$ finito. Então, como não existe nenhuma razão para $f_{2}$, e consequentemente $g_{2}$, anular-se na região $\xi_{0} \leq \xi \leq 1$, o segundo termo em (3.40) é em geral não nulo [78]. Logo, partindo de (3.29) e fazendo o caminho inverso, não recuperamos a expressão original para o momento, (3.17), e o procedimento de Georgi e Politzer [26] parece ser inconsistente. Por outro lado, se as distribuições de pártons forem nulas no intervalo $\xi_{0} \leq \xi \leq 1$, os momentos terão que ser dependentes de $\xi_{0}[78]$ :

$$
\mathcal{A}_{n}\left(\xi_{0}\right) C_{i}^{n}=\int_{0}^{1} d \xi \xi^{n-1} f_{i}\left(\xi ; \xi_{0}\right) \rightarrow \frac{d \mathcal{A}_{n}\left(\xi_{0}\right)}{d \xi_{0}}=\int_{0}^{1} d \xi \xi^{n} \frac{d f_{i}\left(\xi ; \xi_{0}\right)}{d \xi_{0}} \neq 0
$$

implicando que as distribuições de pártons universais (independente do processo) não existem mais para $Q^{2}$ finito e a separação entre distâncias longas e curtas no cone de luz, pela OPE, não é mais possível [78].

Caso a condição de que as funções de estrutura devem ser nulas para $\xi>\xi_{0}$ não for imposta, encontra-se que a energia e momento não são conservadas: quando o 
limite de integração do momento (3.37) é estendido de $x=1$ para $x=\frac{1}{1-\mu}$, o valor máximo para a variável $\xi$ é 1. Assim, os três primeiros termos em (3.39) vão a zero e se recupera o momento (3.17). No entanto, isto implica que para se ter consistência é preciso violar a conservação de energia e momento [78].

Uma verificação numérica da importância da região $\xi>\xi_{0}$ pode ser feita a partir de uma distribuição de pártons simples:

$$
x f_{2}(x)=\frac{35}{32} \sqrt{x}(1-x)^{3}
$$

que reproduz aproximadamente uma típica distribuição de quarks de valência, com normalização $\int_{0}^{1} d x f_{2}(x)=1$. Na Figura (3.5) fez-se uma comparação do momento de Cornwall-Norton (3.17) para vários valores de $j$ (desde o primeiro termo $j=0$ até os primeiros quatro termos $j<4$ ) com a inversão (3.39). As curvas nomeadas $G P$ são aquelas que usam a função de estrutura com TMC que se obtêm com a abordagem utilizada por Georgi e Polizter, equação (3.29): "GP, $\xi_{\max }=\xi_{0}$ " indica que na integral de (3.39) o limite superior foi fixado em $\xi=\xi_{0}$, equação (3.33), respeitando a conservação de energia e momento; e "GP, $\xi_{\max }=1$ " indica que na integral de (3.39) o limite superior foi fixado em $\xi=1$, violando a conservação de energia e momento. Observando a Figura (3.5) vemos uma grande diferença, na região de $Q^{2}$ pequeno, entre as curvas " $G P, \xi_{\max }=\xi_{0}$ " (linha vermelha ponto-ponto-traço) e "GP, $\xi_{\text {max }}=1$ " (linha vermelha contínua) quando comparadas com a curva " $j<4$ " (linha tracejada), sendo assim possível concluir que para obter o momento $M_{2}^{(n=2)}\left(Q^{2}\right)$ é preciso utilizar a região não física $\xi_{0} \leq \xi \leq 1$, na integração de (3.39), que implica em $x>1$.

O problema mencionado acima se encontra no centro da interpretação partônica dos elementos de matriz $\mathcal{A}_{n}$. A abordagem utilizada por Georgi e Politzer [26] tenta manter a interpretação partônica em $Q^{2}$ finito introduzindo uma nova variável de scaling $\xi$. Porém, como foi visto na discussão após a equação (3.39) e pelo estudo numérico mostrado na Figura (3.5), percebe-se que a utilização do scaling $\xi$ leva a 


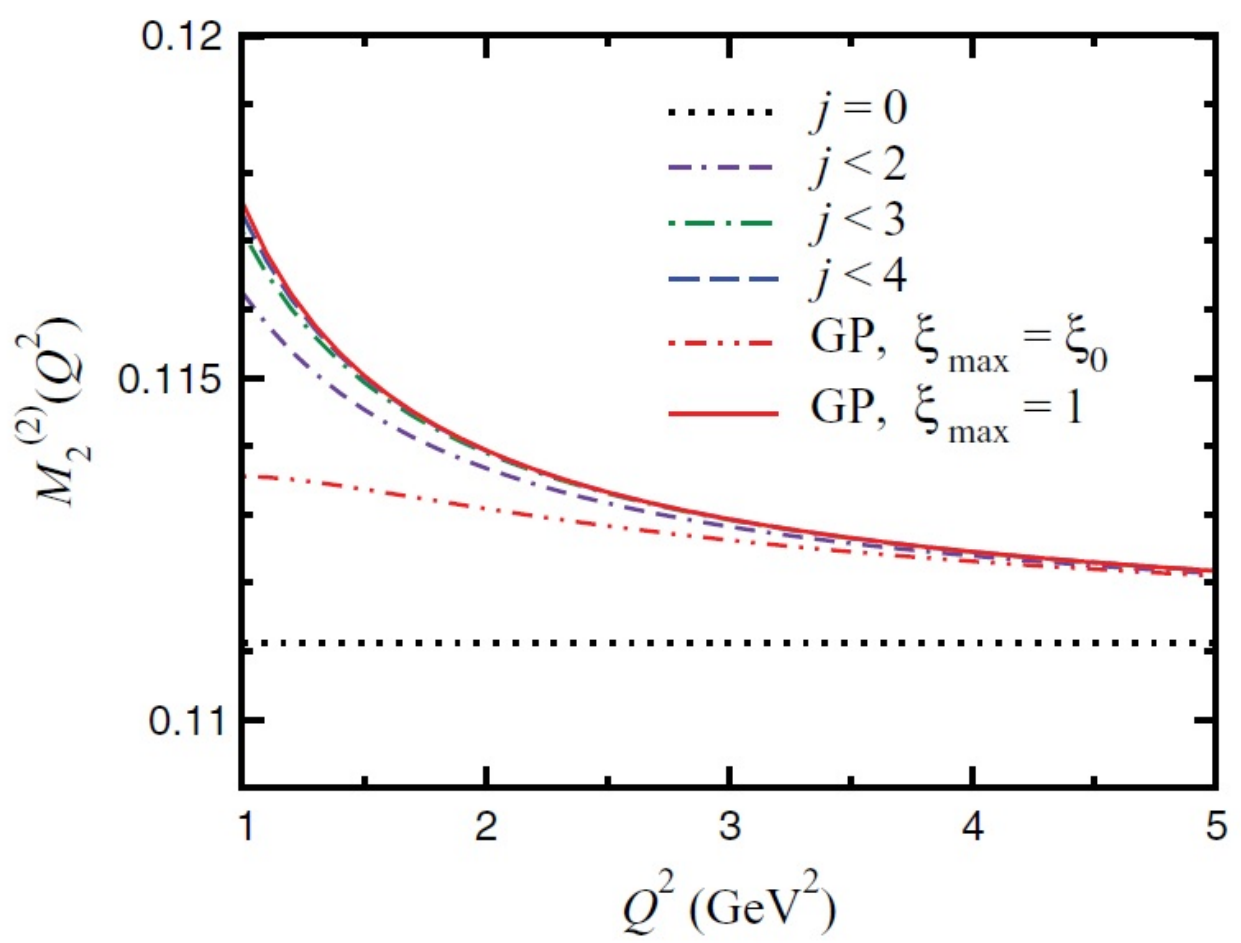

Figura 3.5: $M_{2}^{(n=2)}\left(Q^{2}\right)$ (linhas coloridas), equação (3.17), para $j=0$ (linha pontilhada), $j<2$ (linha ponto-traço-traço), $j<3$ (linha ponto-traço) e $j<4$ (linha tracejada), comparada com a inversão (3.39), sendo o limite superior de integração $\xi_{\max }=\xi_{0}$ (linha vermelha ponto-ponto-traço) e $\xi_{\max }=1$ (linha vermelha contínua). Extraído de [78].

uma inconsistência na extração da dependência em $x$ das funções de estrutura e seus momentos [78]. 


\section{Capítulo 4}

\section{Correções da Massa do Alvo como Expansão em Série}

No capítulo anterior foram revisados os problemas que se encontra ao tentar incorporar as TMC nas funções de estrutura. Particularmente, devido a utilização da variável de scaling $\xi$, equação (3.1), tem-se ou a perda da universalidade da distribuição partônica ou a violação da conservação de energia e momento.

Uma possível solução para este problema é não utilizar a variável de Nachtmann $\xi$. Isto pode ser feito realizando a inversão dos momentos (3.17) e (3.18) ordem por ordem em $\mu$ em vez de somar sobre todas as potências de $\mu$ durante a inversão. Será visto que neste caso se tem a preservação da universalidade das distribuições partônicas e a conservação de energia e momento, mas a região de $x$ e $Q^{2}$ em que se pode ter uma consistência das distribuições partônicas na presença das TMC se torna um tanto restrita.

\subsection{TMC como inversão termo por termo}

Para realizar a inversão dos momentos e obter as funções de estrutura (3.29) e (3.30), Subseção 3.1.2 e Apêndice A, foi utilizado o teorema binomial (equações (3.25), (A.8) e (A.31)) para absorver os fatores combinatórios que envolvem a variável de inte- 
gração $n$, tornando as integrais mais simples de serem resolvidas. Agora iremos realizar um procedimento diferente: em vez de utilizarmos o teorema binomial, vamos inverter os momentos termo por termo, absorvendo os fatores combinatórios em derivadas, que vão resultar em uma expansão em série para as funções de estrutura. Nesta seção será feita a derivação para a função de estrutura $F_{2}$; para as outras funções de estrutura veja o Apêndice B.

Partindo da transformada inversa de Mellin, equação (3.19), para $i=2$ e usando as equações (3.17) e (3.22), temos:

$$
\begin{aligned}
F_{2}^{T M C}\left(x, Q^{2}\right) & =\frac{1}{2 \pi i} \int_{-i \infty}^{i \infty} d n x^{-n+1} M_{2}^{(n)}\left(Q^{2}\right) \\
& =\frac{1}{2 \pi i} \sum_{j=0}^{\infty} \mu^{j} \int_{-i \infty}^{i \infty} d n \int_{0}^{1} d y\left(\begin{array}{c}
n+j \\
j
\end{array}\right) n(n-1) x^{-n+1} y^{n+2 j-2} g_{2}(y) \\
& =\frac{1}{2 \pi i} \sum_{j=0}^{\infty} \mu^{j} \int_{-i \infty}^{i \infty} d n \int_{0}^{1} d y \frac{(n+j) !}{j !(n-2) !} x^{-n+1} y^{n+2 j-2} g_{2}(y)
\end{aligned}
$$

Podemos utilizar a relação:

$$
\frac{(n+j) !}{(n-2) !} x^{-n+1}=(-x)^{2+j} \frac{\partial^{2+j}}{\partial x^{2+j}} x^{-n+1}
$$

para todo $n$ no eixo imaginário, exceto na origem. Substituindo (4.2) em (4.1):

$$
\begin{aligned}
F_{2}^{T M C}\left(x, Q^{2}\right) & =\sum_{j=0}^{\infty} \mu^{j} \frac{(-x)^{2+j}}{j !} \frac{\partial^{2+j}}{\partial x^{2+j}}\left[\frac{1}{2 \pi i} \int_{-i \infty}^{i \infty} d n \int_{0}^{1} d y x^{-n+1} y^{n+2 j-2} g_{2}(y)\right] \\
& =\sum_{j=0}^{\infty} \mu^{j} \frac{(-x)^{2+j}}{j !} \frac{\partial^{2+j}}{\partial x^{2+j}}\left[\int_{0}^{1} d y x y^{2 j-2} g_{2}(y)\right]\left[\frac{1}{2 \pi i} \int_{-i \infty}^{i \infty} d n\left(\frac{y}{x}\right)^{n}\right] \\
& =\sum_{j=0}^{\infty} \mu^{j} \frac{(-x)^{2+j}}{j !} \frac{\partial^{2+j}}{\partial x^{2+j}}\left[\int_{0}^{1} d y x y^{2 j-2} g_{2}(y)\right] \delta\left[\ln \left(\frac{y}{x}\right)\right],
\end{aligned}
$$

onde foi usada a propriedade (3.27). 
Como feito na Subseção 3.1.2, a integral em y é resolvida utilizando a relação (3.28), com $f(y)=\ln \left(\frac{y}{x}\right)$ e $f^{\prime}(y)=\frac{1}{y}$, e a única raíz para $y \in[0,1]$ é $y=x$. Assim, a equação (4.3) é reescrita como:

$$
\begin{aligned}
& F_{2}^{T M C}\left(x, Q^{2}\right)=\sum_{j=0}^{\infty} \mu^{j} \frac{(-x)^{2+j}}{j !} \frac{\partial^{2+j}}{\partial x^{2+j}}\left[\int_{0}^{1} d y x y^{2 j-2} g_{2}(y) x \delta(y-x)\right] \\
& F_{2}^{T M C}\left(x, Q^{2}\right)=x^{2} \sum_{j=0}^{\infty} \mu^{j} \frac{(-x)^{j}}{j !} \frac{\partial^{2+j}}{\partial x^{2+j}}\left[x^{2 j} g_{2}(x)\right] .
\end{aligned}
$$

A equação (4.4) pode ser obtida, também, usando:

$$
\frac{(n+j) !}{(n-2) !} y^{n+2 j-2}=y^{2 j} \frac{\partial^{2+j}}{\partial y^{2+j}} y^{n+j}
$$

e substituindo em (4.1):

$$
\begin{aligned}
F_{2}^{T M C}\left(x, Q^{2}\right) & =\sum_{j=0}^{\infty} \mu^{j} \frac{1}{j !} \frac{1}{2 \pi i} \int_{-i \infty}^{i \infty} d n \int_{0}^{1} d y x^{-n+1} g_{2}(y) y^{2 j} \frac{\partial^{2+j}}{\partial y^{2+j}}\left[y^{n+j}\right] \\
& =\sum_{j=0}^{\infty} \mu^{j} \frac{x}{j !} \int_{0}^{1} d y g_{2}(y) y^{2 j} \frac{\partial^{2+j}}{\partial y^{2+j}}\left[y^{j} \frac{1}{2 \pi i} \int_{-i \infty}^{i \infty} d n\left(\frac{y}{x}\right)^{n}\right] \\
& =\sum_{j=0}^{\infty} \mu^{j} \frac{x}{j !} \int_{0}^{1} d y g_{2}(y) y^{2 j} \frac{\partial^{2+j}}{\partial y^{2+j}}\left\{y^{j} \delta\left[\ln \left(\frac{y}{x}\right)\right]\right\} \\
& =\sum_{j=0}^{\infty} \mu^{j} \frac{x}{j !} \int_{0}^{1} d y g_{2}(y) y^{2 j} \frac{\partial^{2+j}}{\partial y^{2+j}}\left[y^{j} x \delta(y-x)\right] \\
& =\sum_{j=0}^{\infty} \mu^{j} \frac{x^{2}}{j !} \int_{0}^{1} d y g_{2}(y) y^{2 j} \frac{\partial^{2+j}}{\partial y^{2+j}}\left[y^{j} \delta(y-x)\right]
\end{aligned}
$$

onde também foram usadas a propriedade (3.27) e a relação (3.28). Como a função $\delta$ é uma distribuição, tem-se a relação (análoga à integração por partes de uma função 
regular) para dada função $\phi$ :

$$
\int d y \delta(y) \phi^{\prime}(y)=-\int d y \delta^{\prime}(y) \phi(y)
$$

Utilizando a relação (4.7) $2+j$ vezes em (4.6), tem-se a equação (4.4):

$$
\begin{aligned}
& F_{2}^{T M C}\left(x, Q^{2}\right)=\sum_{j=0}^{\infty} \mu^{j} \frac{(-1)^{2+j} x^{2}}{j !} \int_{0}^{1} d y y^{j} \delta(y-x) \frac{\partial^{2+j}}{\partial y^{2+j}}\left[y^{2 j} g_{2}(y)\right] \\
& F_{2}^{T M C}\left(x, Q^{2}\right)=x^{2} \sum_{j=0}^{\infty} \mu^{j} \frac{(-x)^{j}}{j !} \frac{\partial^{2+j}}{\partial x^{2+j}}\left[x^{2 j} g_{2}(x)\right] .
\end{aligned}
$$

Seguindo qualquer um dos passos para encontrar as análogas da equação (4.4) para as outras funções de estrutura, tem-se (a dedução detalhada encontra-se no Apêndice $\mathrm{B})$ :

$$
\begin{aligned}
& F_{1}^{T M C}\left(x, Q^{2}\right)=x \sum_{j=0}^{\infty} \mu^{j} \frac{(-x)^{j}}{j !} \frac{\partial^{j}}{\partial x^{j}}\left[x^{2 j-2}\left(\frac{x}{2} f_{1}(x)+j g_{2}(x)\right)\right] \\
& F_{L}^{T M C}\left(x, Q^{2}\right)=x^{2} \sum_{j=0}^{\infty} \mu^{j} \frac{(-x)^{j}}{j !} \frac{\partial^{j}}{\partial x^{j}}\left[x^{2 j-2}\left(x f_{2}(x)-x f_{1}(x)+4 j g_{2}(x)\right)\right] ; \\
& F_{3}^{T M C}\left(x, Q^{2}\right)=\sum_{j=0}^{\infty} \mu^{j} \frac{(-x)^{1+j}}{j !} \frac{\partial^{1+j}}{\partial x^{1+j}}\left[x^{2 j} h_{3}(x)\right] \\
& F_{4}^{T M C}\left(x, Q^{2}\right)= \\
& \sum_{j=0}^{\infty} \mu^{j} \frac{(-x)^{j}}{j !} \frac{\partial^{j}}{\partial x^{j}}\left[x ^ { 2 j - 2 } \left(j(j-1) g_{2}(x)\right.\right. \\
& F_{5}^{T M C}\left(x, Q^{2}\right)=\sum_{j=0}^{\infty} \mu^{j} \frac{(-x)^{1+j}}{j !} \frac{\partial^{1+j}}{\partial x^{1+j}}\left[x^{2 j-1}\left(-j g_{2}(x)+\frac{x}{2} h_{5}(x)\right)\right]
\end{aligned}
$$

Observe que a variável de Nachtmann $\xi$ não aparece em nenhuma das equações (4.4) e (4.9), sendo as distribuições $f_{i}, h_{i}$ e $g_{i}$ dependentes somente da variável $x$ (como 
requerido). O termo $j=0$ na expansão em série (4.4) e (4.9) representa as funções de estrutura no limite sem massa, como esperado.

Com esta formulação, evitamos os problemas encontrados com a consistência, devido à variável $\xi$, mostrado na Subseção 3.3.1. Isto pode ser verificado seguindo o mesmo procedimento da Subseção 3.3.1, fazendo a substituição de (4.8) em (2.35), com $i=2$ :

$$
\begin{aligned}
M_{2}^{(n)}\left(Q^{2}\right)= & \int_{0}^{1} d x x^{n-2} F_{2}^{T M C}\left(x, Q^{2}\right) \\
= & \sum_{j=0}^{\infty} \frac{\mu^{j}}{j !}(-1)^{j} \int_{0}^{1} d x x^{n+j} \frac{\partial^{2+j}}{\partial x^{2+j}}\left[x^{2 j} g_{2}(x)\right] \\
= & \sum_{j=0}^{\infty} \frac{\mu^{j}}{j !}(-1)^{j}\left\{\left.\left[x^{n+j} \frac{\partial^{1+j}}{\partial x^{1+j}}\left[x^{2 j} g_{2}(x)\right]\right]\right|_{0} ^{1}\right. \\
& \left.\quad-(n+j) \int_{0}^{1} d x x^{n+j-1} \frac{\partial^{1+j}}{\partial x^{1+j}}\left[x^{2 j} g_{2}(x)\right]\right\} .
\end{aligned}
$$

Como a função $g_{2}(x)$ se anula para $x=0$ e $x=1$, o primeiro termo de (4.10) é zero, e repetindo $1+j$ vezes o procedimento acima, temos:

$$
\begin{aligned}
M_{2}^{(n)}\left(Q^{2}\right) & =\sum_{j=0}^{\infty} \frac{\mu^{j}}{j !}(-1)^{j}\left[(-1)^{2+j} \frac{(n+j) !}{(n-2) !} \int_{0}^{1} d x x^{n+2 j-2} g_{2}(x)\right] \\
& =\sum_{j=0}^{\infty} \frac{(n+j) !}{j ! n !} \mu^{j} n(n-1) \int_{0}^{1} d x x^{n+2 j-2} g_{2}(x) .
\end{aligned}
$$

Substituindo (3.22) em (4.11) e utilizando $\left(\begin{array}{l}n \\ k\end{array}\right)=\frac{n !}{k !(n-k) !}$, chega-se em:

$$
M_{2}^{(n)}\left(Q^{2}\right)=\sum_{j=0}^{\infty}\left(\begin{array}{c}
n+j \\
j
\end{array}\right) \mu^{j} \frac{n(n-1)}{(n+2 j)(n+2 j-1)} C_{2}^{n+2 j} \mathcal{A}_{n+2 j}
$$

que é o momento (3.17), mostrando a consistência de (4.8). 


\subsection{Convergência das séries}

Para estudarmos a convergência da série, equações (4.8) e (4.9), utilizamos a PDF dada pela MSTW 2008 [16].

Na Figura (4.1) são mostradas os primeiros termos da série (4.8), desde o primeiro $(j=0)$ até o quarto $(j<5)$ termo. Para comparação também são mostradas as TMC calculadas na Subseção 3.1.2, equação (3.29), chamada na figura de GP. Verifica-se que a convergência para $Q^{2}=1 \mathrm{GeV}^{2}$ é bem rápida para aproximadamente $x \lesssim 0.5$, sendo os três primeiros termos suficientes para uma boa convergência. Vale ressaltar que para estes valores de $x$ e $Q^{2}$ já estamos dentro da região de ressonância do nucleon $(W<2 \mathrm{GeV})$, a qual começa para $Q^{2}=1 \mathrm{GeV}^{2}$ em $x \gtrsim 0.24$. A série é bem controlada até praticamente o pico da ressonância $\Delta(1232), x \simeq 0.61$.

A região de ressonância pode ser melhor visualizada a partir da Figura (4.2), onde $F_{2}$ é mostrada em função de $W$. Nota-se que até $W \approx 1.35 \mathrm{GeV}$ a série é bem comportada, pouco antes do pico da ressonância $\Delta(1232)$. Verifica-se que tanto para baixo $W$, na Figura (4.2), ou alto $x$, na Figura (4.1), os termos superiores em $j$ apresentam um comportamento oscilatório próximo do ponto elástico, $x=1$ ou $W=$ $m_{p}$. Os três primeiros termos $(j<3)$ são nulos para $x \rightarrow 1$, enquanto os de ordem mais alta $(j>3)$ divergem para $x \rightarrow 1$, o anulamento esta relacionado com a forma da PDF utilizada que é proporcional a $(1-x)^{3}$. Também se vê que a TMC dada pela equação (3.29), abordagem de Georgi e Politzer, é finita em $x=1$ e assim leva a regiões não físicas $\left(W<m_{p}\right)$.

Nas Figuras (4.3) são mostradas a razão entre a $F_{2}$ dada pela equação $(4.8)$ e a $F_{2}$ calculada seguindo Georgi e Politzer, em função de $x$ e de $W$. A análise através da razão das funções é interessante pois assim podemos perceber com uma maior clareza qual é o ponto máximo de convergêcia com determinado número de termos das séries obtidas na Subseção 4.1. Na Tabela 4.1 mostramos essa evolução. Note, na figura de baixo (função de $W$ ), o anulamento da razão entre o termo com $j=0$ e a $F_{2}^{G P}$ quando $W \rightarrow m_{p}$, isto reflete o valor não nulo de $F_{2}^{G P}$ para $x \geq 1$. 
Tabela 4.1: Convergência máxima de $F_{2} \operatorname{com} Q^{2}=1 G e V^{2}$ com diferentes $j$

\begin{tabular}{c|c}
\hline$j$ & $x_{\max }$ \\
\hline \hline$<2$ & $\simeq 0.25$ \\
\hline$<3$ & $\simeq 0.30$ \\
\hline$<4$ & $\simeq 0.35$ \\
\hline$<5$ & $\simeq 0.50$ \\
\hline
\end{tabular}

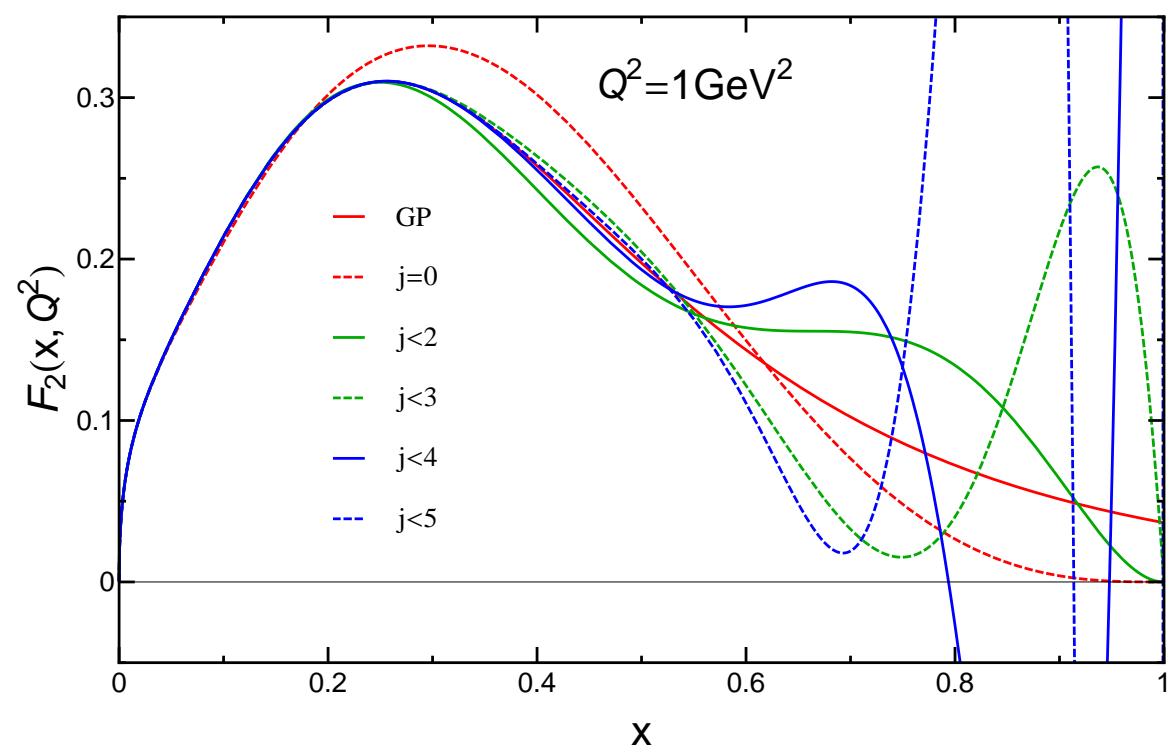

Figura 4.1: TMC para $F_{2}$ em $Q^{2}=1 G e V^{2}$ pela equação (4.8), mostrando a convergência com o aumento de $j$, comparada com o resultado da equação (3.29), em função de $x$.

A oscilação na região de $x$ grande é suavizada quando $Q^{2}$ cresce, como pode ser visto nas Figuras (4.4). Claramente se percebe que para $Q^{2}=5 \mathrm{GeV}^{2}$ praticamente não se observa oscilação e, como antes, os três primeiros termos $(j<3)$ são suficientes para uma boa convergência até aproximadamente $x \simeq 0.75$ ou $W \simeq 1.6 \mathrm{GeV}$, sendo que a ressonância $\Delta(1232)$ encontra-se em $x \simeq 0.89\left(\right.$ para $\left.Q^{2}=5 G e V^{2}\right)$. A diminuição na oscilação quando se aumenta $Q^{2}$ é esperada, pois ela foi causada pela adição das TMC nas funções de estrutura e como as correções são inversamente proporcionais a $Q^{2}$, os efeitos dessa correção passam a ser desprezíveis.

Na Figura (4.5) são mostradas as TMC para $F_{2} \operatorname{com} Q^{2}=2 \mathrm{GeV}^{2}$ para valores de $j$ muito grande $(j>100)$. Por conveniência foi utilizada a distribuição dada pela 


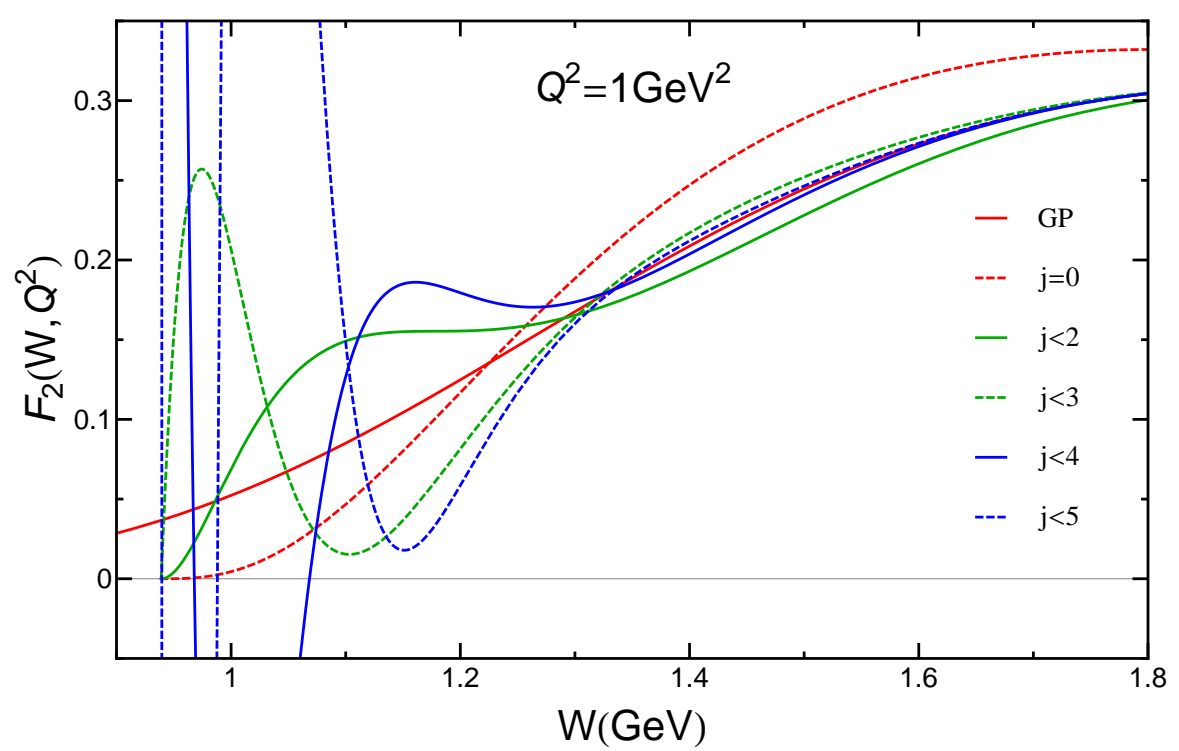

\section{Figura 4.2: $O$ mesmo que a Figura (4.1), mas em função de $W$ (massa do estado hadrônico final).}

equação (3.42). Vê-se que existe convergência para $x \lesssim 0.744$, que corresponde a $W \gtrsim 1.253 \mathrm{GeV}$ (valores já bem dentro da região de ressonância do nucleon, para este $Q^{2}$ o pico de $\Delta(1232)$ está em $\left.x \simeq 0.759\right)$. Vale ressaltar que a adição de números pares ou ímpares em $j$ resulta em comportamentos divergentes alternando entre negativo e positivo respectivamente. Para conseguirmos uma convergência para valores maiores de $x$ devemos adicionar mais termos na série, porém o processo torna-se inviável quando se compara o avanço em $x$ com a quantidade de termos necessários para convergência. A Tabela 4.2 resume o valor máximo de $x$ que encontramos ao analisar a Figura (4.5). Isto parece indicar a existência de um $x$ máximo para a convergência,

Tabela 4.2: Convergência máxima de $F_{2} \operatorname{com} Q^{2}=2 G e V^{2}$ com diferentes $j$

\begin{tabular}{c|c}
\hline$j$ & $x_{\max }$ \\
\hline \hline$<101$ & $\simeq 0.735$ \\
\hline$<201$ & $\simeq 0.741$ \\
\hline$<301$ & $\simeq 0.744$ \\
\hline
\end{tabular}

sendo neste caso um valor entre $0.74 \lesssim x \lesssim 0.75$. É provável que o valor exato deste ponto dependa da forma específica da PDF usada $\left(\propto(1-x)^{3}\right)$, embora não tenhamos 

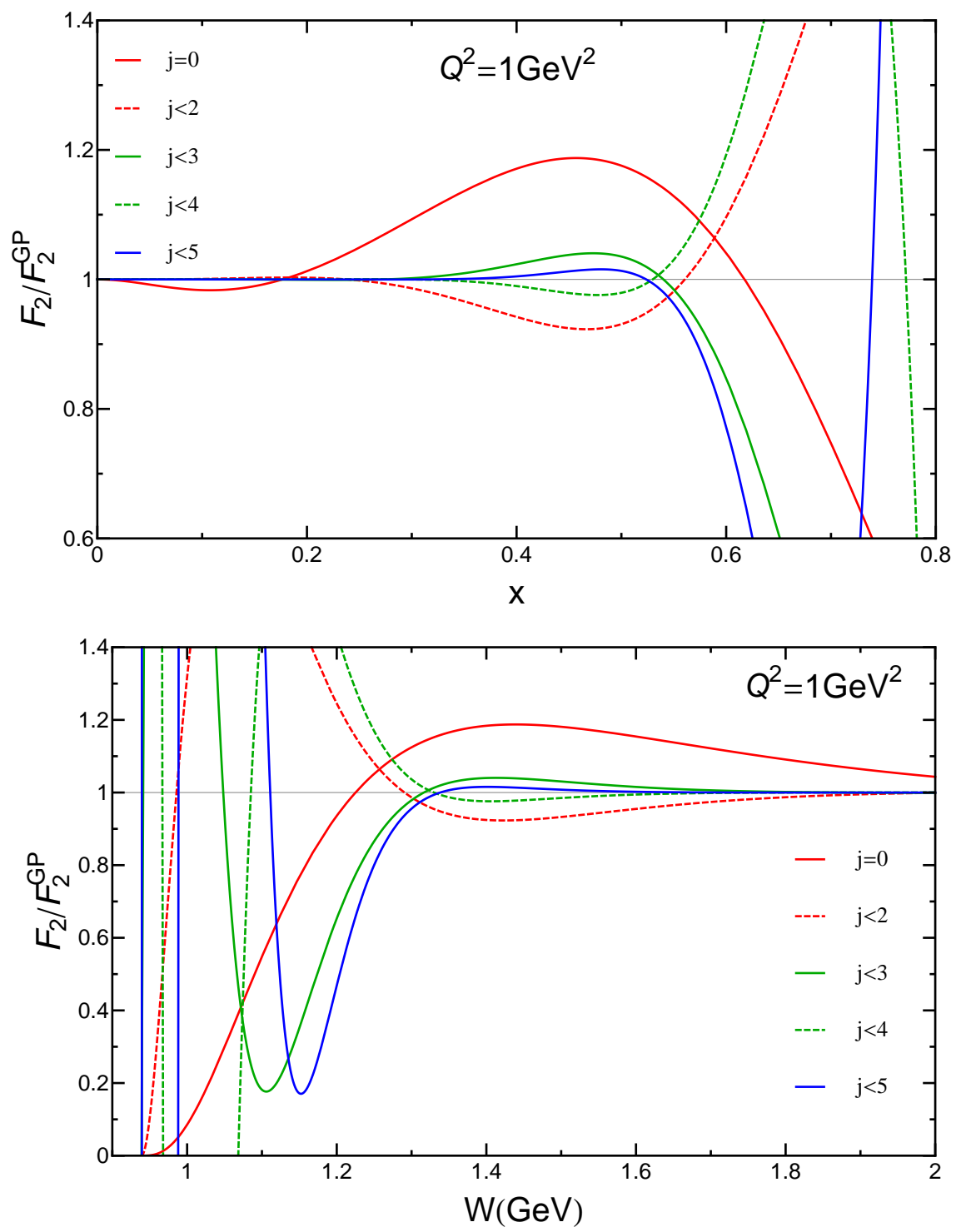

Figura 4.3: Razão entre a TMC $F_{2} \operatorname{com} Q^{2}=1 G e V^{2}$ dada por (4.8) para vários $j(j=0$ até $j<5)$ com a TMC (3.29), em função de $x$ (acima) e de $W$ (abaixo).

verificado esta afirmação.

Nas Figuras em (4.6) tem-se a razão entre a série para $F_{2}$ e a equação (3.29) em função de $x$ e $W$ para $Q^{2}=2 G e V^{2}$, foi usada a PDF MSTW 2008 [16]. Neste caso com o primeiro termo $(j<2)$ obtemos $x_{\text {max }}^{j<2} \simeq 0.49$, com os dois primeiros termos $(j<3) x_{\text {max }}^{j<3} \simeq 0.5$, com os três primeiros termos $(j<4) x_{\text {max }}^{j<4} \simeq 0.54$ e com os quatro primeiros termos $(j<5) x_{\max }^{j<5} \simeq 0.7$. As Figuras de $(4.7)$ mostram $F_{2}$ em função de $x$ 

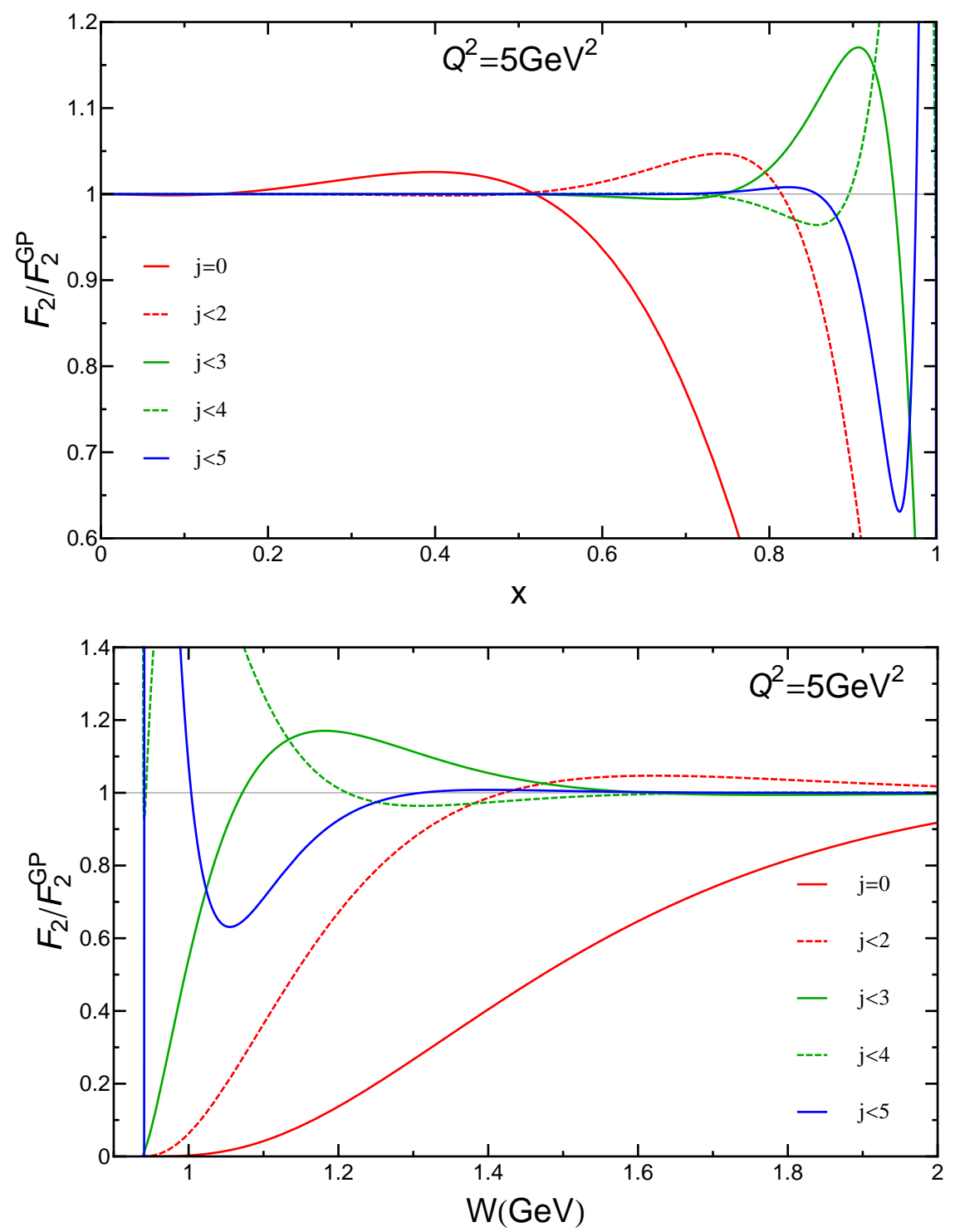

Figura 4.4: Razão entre a TMC $F_{2}$ com $Q^{2}=5 G e V^{2}$ dada por (4.8) para vários $j(j=0$ até $j<5)$ com a TMC (3.29), em função de $x$ (acima) e de $W$ (abaixo).

e $W$ para $Q^{2}=2 G e V^{2}$ e $Q^{2}=5 G e V^{2}$.

Analisando as Figuras (4.1) e (4.2) juntamente com (4.7) percebe-se a atenuação das oscilações quando $x \rightarrow 1$ ou $W \rightarrow m_{p}$, conforme $Q^{2}$ aumenta (já que para $Q^{2} \rightarrow \infty$, limite de Bjorken, o efeito das TMC são desprezíveis).

É importante verificar se o ponto em $x$ e $Q^{2}$ a partir do qual as séries divergem depende de uma função de estrutura em particular. Assim, estudamos a convergência 


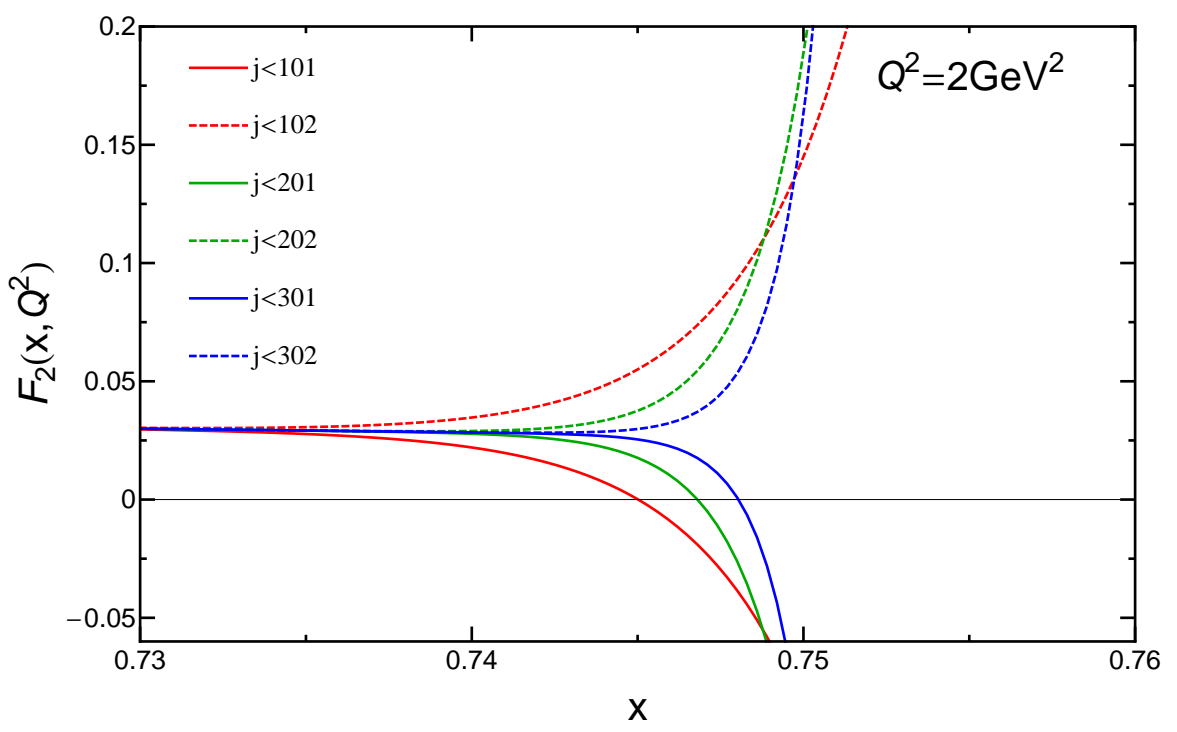

Figura 4.5: TMC para $F_{2}$ em $Q^{2}=2 G e V^{2}$ pela equação (4.8), mostrando a convergência para grandes valores de $j<101,102,201,202,301$ e 302, em função de $x$.
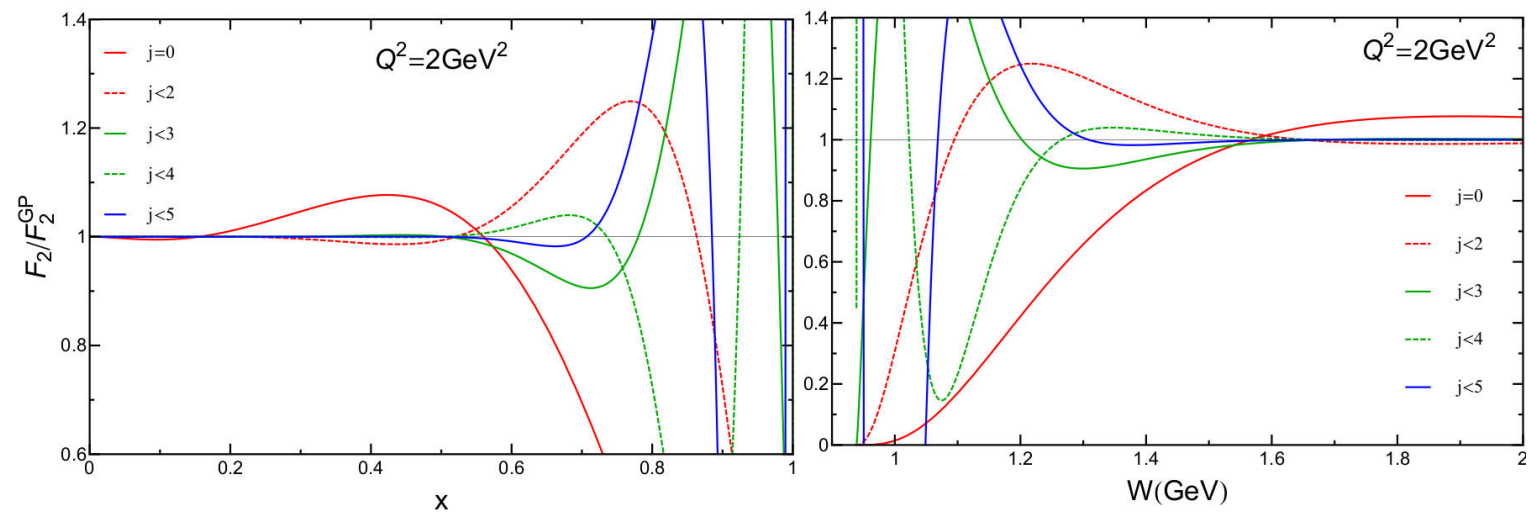

Figura 4.6: Razão entre a TMC $F_{2}$ com $Q^{2}=2 G e V^{2}$ dada por (4.8) para vários $j$ com a TMC (3.29), em função de $x$ (esquerda) e de $W$ (direita).

das funções de estrutura $F_{1}$ e $F_{3}$ nas Subseções B.1.1 e B.3.1, respectivamente. Na Tabela 4.3 é mostrado um resumo das regiões em $x$ e $W$ onde se tem convergência, com os três primeiros termos da soma, para diferentes valores de $Q^{2}$. Vê-se da tabela que a convergência é alcançada sempre dentro da região de ressonância do nucleon, $W \lesssim$ $2 \mathrm{GeV}$ e nas proximidades do pico da ressonância $\Delta(1232)$. Isto implica que as análises globais, que foram feitas em $W>2 \mathrm{GeV}$, não sofrem nenhum impacto: CTEQ6 

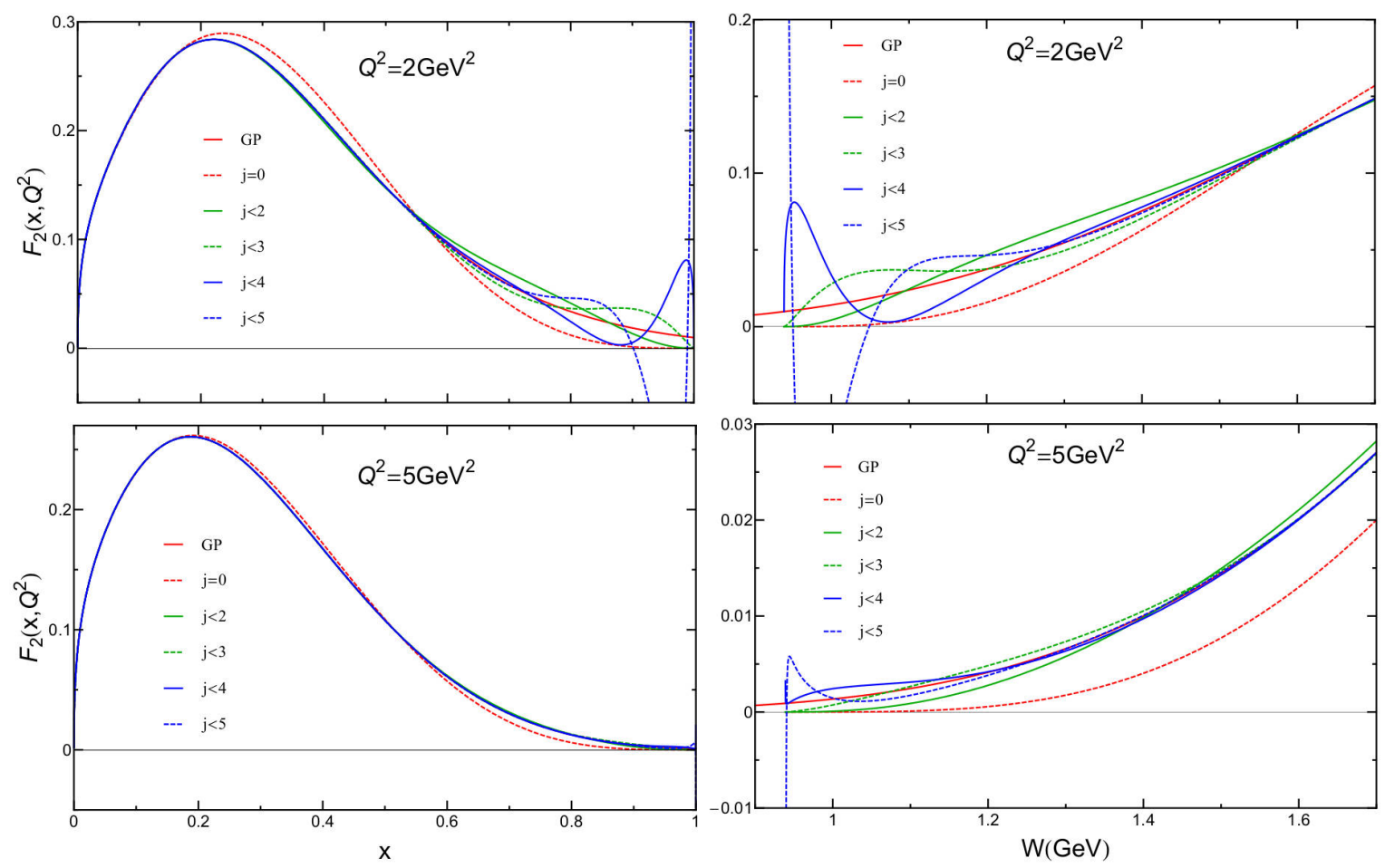

Figura 4.7: TMC para $F_{2}$ em $Q^{2}=2 G e V^{2}$ (acima) e $Q^{2}=5 G e V^{2}$ (abaixo) pela equação (4.8), mostrando a convergência com o aumento de $j$, comparada com o resultado da equação (3.29), em função de $x$ (esquerda) e $W$ (direita).

$W \geq 3.5 \mathrm{GeV}$ [14], GRV/GJR09 $W \geq 3.16 \mathrm{GeV}$ [15], MSTW08 $W \geq 3.87 \mathrm{GeV}$ [16], ABKM09 $W \geq 3.5 \mathrm{GeV}[17]$ e a mais recente CJ12 (e menos restritiva) $W \geq 1.73 \mathrm{GeV}$ $[18]$.

Tabela 4.3: Convergência da série para diferentes $Q^{2}$

\begin{tabular}{c||c|c|c}
\hline & $Q^{2}=1 \mathrm{GeV}^{2}$ & $Q^{2}=2 \mathrm{GeV}^{2}$ & $Q^{2}=5 \mathrm{GeV}^{2}$ \\
\hline \hline$F_{1}$ & $x \approx 0.45$ & $x \approx 0.65$ & $x \approx 0.85$ \\
& $W \approx 1.45 \mathrm{GeV}$ & $W \approx 1.4 \mathrm{GeV}$ & $W \approx 1.33 \mathrm{GeV}$ \\
\hline$F_{2}$ & $x \approx 0.50$ & $x \approx 0.54$ & $x \approx 0.75$ \\
& $W \approx 1.37 \mathrm{GeV}$ & $W \approx 1.61 \mathrm{GeV}$ & $W \approx 1.60 \mathrm{GeV}$ \\
\hline$F_{3}$ & $x \approx 0.50$ & $x \approx 0.70$ & $x \approx 0.75$ \\
& $W \approx 1.37 \mathrm{GeV}$ & $W \approx 1.32 \mathrm{GeV}$ & $W \approx 1.60 \mathrm{GeV}$ \\
\hline
\end{tabular}




\section{Capítulo 5}

\section{Conclusões}

Neste trabalho nos propusemos a estudar o problema das TMC nas funções de estrutura, que é tão antigo quanto a própria teoria da QCD. Como o foco principal das análises das funções de estrutura é a região perturbativa, onde os efeitos de correções proporcionais a $\frac{1}{Q^{2}}$ são desprezados, o estudo das TMC foi durante muito tempo ignorado. Com o surgimento de dados na região de transição entre scaling e ressonância, alto $x$ e pequeno $Q^{2}$, o problema das TMC se tornou relevante novamente, dando uma certa urgência para a resolução desta questão.

Nesta dissertação, dedicamos o Capítulo 1 para mostrar a evolução do conceito da estrutura interna do nucleon: partindo do espalhamento simples $e+\mu \rightarrow e^{\prime}+\mu^{\prime}$, passando pelos espalhamentos $e+p$ elástico e inelástico, modelo de pártons e QCD, e mostrando que o nucleon possui uma estrutura interna complexa e em certo grau desconhecida. O Capítulo 2 revisou a OPE que foi largamente utilizada nos capítulos seguintes.

Na Seção 3.1 deduzimos detalhadamente as funções de estrutura com TMC utilizando o formalismo de Georgi e Politzer e, nas Seções subsequentes, realizamos uma análise do problema inerente a nova variável de scaling $\xi$, na região em que $x \rightarrow 1$, e a inversão das funções de estrutura para seus momentos. Foi feito um estudo crítico na definição de PDFs na presença das TMC e discutimos a violação da conservação de energia e momento na abordagem usual, além de mostrar algumas tentativas frus- 
tradas de se resolver este problema. Historicamente é argumentado que este problema existe porque na região de pequeno $Q^{2}$ é preciso considerar os efeitos twists maiores. Porém, acreditamos que é preciso se ter uma formulação consistente twist por twist.

Para evitarmos os problemas encontrados ao se utilizar a variável $\xi$, nos propusemos a não utilizá-la. A derivação detalhada está no Capítulo 4 para a $F_{2}$ e no Apêndice B para todas as outras funções de estrutura, onde as obtivemos como uma série em $\mu=\frac{m_{p}^{2}}{Q^{2}}$ e com as PDFs dependendo somente de $x$. O mérito desta abordagem é que se tem consistência na inversão de seus momentos, sem a necessidade de incluir regiões fisicamente proibidas, onde se tem violação da conservação de energia e momento. Ademais, nos permite estudar sistematicamente as regiões de $x$ e $Q^{2}$ onde as TMC podem ser utilizadas com segurança. Utilizando a PDF MSTW 2008 estudamos a convergência das funções de estrutura $F_{2}$ (Seção 4.2), $F_{1}$ (Subseção B.1) e $F_{3}$ (Subseção B.3), verificando que com apenas os primeiros três termos obtemos uma boa convergência para valores de $x$ ou $W$ sempre na região de ressonância do nucleon, $W \lesssim 2 G e V$, e nas proximidades do pico da ressonância $\Delta(1232)$. Nossa análise sugere que as PDFs não podem ser definidas consistentemente em qualquer $Q^{2}$ quando se incorpora a massa do alvo, mas que é possível calcular a dependência em $x$ das funções de estrutura com TMC como uma série cuja sua convergência pode ser estudada como função de $x$ e $Q^{2}$.

É observado, quando se inclui as TMC nas funções de estrutura, que para grandes valores de $x$ (ou pequenos valores de $W$ ) em um $Q^{2}$ fixo, tem-se grandes oscilações próximo do limite elástico $\left(x=1\right.$ ou $\left.W=m_{p}\right)$, onde se torna muito difícil o controle da série para um número finito de termos. Como exemplo, mostramos o caso para $Q^{2}=2 \mathrm{GeV}^{2}$, somando os primeiros 300 termos obtivemos uma convergência até $x \approx 0.744$ ou $W \approx 1.253 \mathrm{GeV}$. Esta análise nos leva a acreditar na existência de um $x$ máximo, sendo este valor entre $0.74 \lesssim x \lesssim 0.75$ para $Q^{2}=2 G e V^{2}$ e devido a forma complexa da série utilizada, este valor aparenta poder ser calculado somente numericamente. Como mostramos no final da Seção $4.2 \mathrm{um} W$ tão pequeno não é utilizado nas análises globais para o DIS e em prática não impõe sérias restrições nessas 
análises. Nossos resultados fornecem um grande suporte às análises que incorporam dados em $W$ pequeno, com planos de se estender para regiões de ressonância, como exemplo a análise CJ12 [18] que considera dados a partir de $W \geq 1.73 \mathrm{GeV}$.

Como perspectiva pretende-se dar continuidade a esse trabalho fazendo os cálculos das séries com outras PDFs para verificarmos como é o comportamento da convergência em relação a distribuição usada, implementar as TMC nas funções de estrutura com spin e o cálculo das TMC considerando twists maiores.

A compreensão da região de grande $x$ em conjunto com os dados experimentais implicará em restrições nas modelagens para as funções de estrutura. Como é apontado em [94], onde o autor apresenta três modelos $[95,96,97]$ para a razão entre a $F_{2}$ do nêutron com a do próton que divergem para grande $x$. Assim, com os dados futuros espera-se refinar os modelos e eliminar este problema, que precisará considerar as TMC.

Existe uma grande incerteza nas distribuições de glúons para grande $x(x \gtrsim 0.1)$ entre as análises globais, essa discrepância é resultado das diferentes escolhas de dados entre os grupos [22] e que sua produção vem dos quarks de valência que possuem muita incerteza, contribuindo na dubiedade desses glúons. Espera-se que com os dados produzidos pelos aceleradores de última geração reduzam essas incertezas [98].

A região de grande $x$ e pequeno $Q^{2}$ é de suma importância para o estudo da região de pequeno $x$ e grande $Q^{2}$ quando se usa as equações de evolução da QCD, as equações DGLAP [13], pois uma grande incerteza na região de grande $x$ e pequeno $Q^{2}$ afeta, por evolução, a região de pequeno $x$ e grande $Q^{2}[22,21]$.

Vemos que o estudo dessa região ainda necessita de muito esforço teórico e com os projetos experimentais que já estão em andamento e os que estão por vir, nos dará uma visão melhor de como a estrutura interna do nucleon está organizada. 


\section{Apêndice A}

\section{Funções de Estrutura com}

\section{Abordagem de Georgi e Politzer}

Neste apêndice será realizada a derivação das funções de estrutura $F_{i}^{T M C}\left(x, Q^{2}\right)$, com $i=1, L, 3,4,5$, seguindo os procedimentos adotados para a derivação na Seção 3.1 , onde foi feito o cálculo para $F_{2}^{T M C}\left(x, Q^{2}\right)$.

\section{A.1 $F_{1}^{T M C}\left(x, Q^{2}\right)$, Georgi e Politzer}

Primeiro é preciso encontrar $T_{1}$, que é o coeficiente de $-g^{\mu \nu}$ em (3.8), que terá contribuição integral do termo $C_{1}^{2 k}$ e parcial do termo $C_{2}^{2 k}$, devido a identidade algébrica $\left(g_{\mu_{1}}^{\mu} g_{\mu_{2}}^{\nu} Q^{2} C_{2}^{2 k}\right)\left(g^{\mu_{1} \mu_{2}}\right)=g^{\mu \nu} Q^{2} C_{2}^{2 k}$.

Para $C_{1}^{2 k}$, fixando $k \in \mathcal{N}$ e $j \in\{0, \ldots, k\}$, com cada termo de $\{g \ldots g\}\{p \ldots p\}$, encontra-se que $j q_{\mu_{i}}$ terão seus índices levantados por $j g^{\mu_{i} \mu_{l}}$, que vão se contrair com $j q_{\mu_{l}}$ resultando em $\left(q^{2}\right)^{j}$. Os $(2 k-2 j) q_{\mu_{i}}$ irão contrair com os $(2 k-2 j) p^{\mu_{i}}$ dando $(p \cdot q)^{2 k-2 j}$ :

$$
\sum_{k=1}^{\infty}\left(-g^{\mu \nu} q_{\mu_{1}} q_{\mu_{2}} C_{1}^{2 k}\right) q_{\mu_{3}} \ldots q_{\mu_{2 k}} \frac{2^{2 k}}{\left(Q^{2}\right)^{2 k}} \mathcal{A}_{2 k} \Pi^{\mu_{1} \ldots \mu_{2 k}}=
$$




$$
\begin{aligned}
& =-g^{\mu \nu} \sum_{k=1}^{\infty} \frac{2^{2 k}}{\left(Q^{2}\right)^{2 k}} \sum_{j=0}^{k}(-1)^{j} \frac{(2 k-j) !}{2^{j}(2 k) !} \frac{(2 k) !}{2^{j} j !(2 k-2 j) !}\left(p^{2} q^{2}\right)^{j} \\
& \times(p \cdot q)^{2 k-2 j} C_{1}^{2 k} \mathcal{A}_{2 k}
\end{aligned}
$$

Para $C_{2}^{2 k}$, fixando $k \in \mathcal{N}$ e $j \in\{0, \ldots, k\}$, com os termos de $\{g \ldots g\}\{p \ldots p\}$ que possuem $g^{\mu_{1} \mu_{2}}$, assim é preciso fazer $j \rightarrow j-1$ e $k \rightarrow k-1$, com o mesmo raciocínio usado em $C_{1}^{2 k}$, chega-se:

$$
\begin{aligned}
& \sum_{k=1}^{\infty}\left(g_{\mu_{1}}^{\mu} g_{\mu_{2}}^{\nu} Q^{2} C_{2}^{2 k}\right) q_{\mu_{3}} \ldots q_{\mu_{2 k}} \frac{2^{2 k}}{\left(Q^{2}\right)^{2 k}} \mathcal{A}_{2 k} \Pi^{\mu_{1} \ldots \mu_{2 k}}= \\
& =\quad g^{\mu \nu} \sum_{k=1}^{\infty} \frac{2^{2 k}}{\left(Q^{2}\right)^{2 k}} \sum_{j=1}^{k}(-1)^{j} \frac{(2 k-j) !}{2^{j}(2 k) !} \frac{(2 k-2) !}{2^{j-1}(j-1) !(2 k-2 j) !}\left(p^{2}\right)^{j}\left(q^{2}\right)^{j-1} \\
& \times \quad(p \cdot q)^{2 k-2 j} Q^{2} C_{2}^{2 k} \mathcal{A}_{2 k}+\text { termos sem } g^{\mu_{1} \mu_{2}}
\end{aligned}
$$

Então com (A.1) e (A.2):

$$
\begin{aligned}
T_{1}\left(x, Q^{2}\right) & =\sum_{k=1}^{\infty} \frac{2^{2 k}}{\left(Q^{2}\right)^{2 k}} \sum_{j=0}^{k}(-1)^{j} \frac{(2 k-j) !}{2^{j}(2 k) !} \frac{(2 k) !}{2^{j} j !(2 k-2 j) !}\left(p^{2} q^{2}\right)^{j}(p \cdot q)^{2 k-2 j} C_{1}^{2 k} \mathcal{A}_{2 k} \\
& -\sum_{k=1}^{\infty} \frac{2^{2 k}}{\left(Q^{2}\right)^{2 k}} \sum_{j=1}^{k}(-1)^{j} \frac{(2 k-j) !}{2^{j}(2 k) !} \frac{(2 k-2) !}{2^{j-1}(j-1) !(2 k-2 j) !} \\
& \times\left(p^{2}\right)^{j}\left(q^{2}\right)^{j-1}(p \cdot q)^{2 k-2 j} Q^{2} C_{2}^{2 k} \mathcal{A}_{2 k}
\end{aligned}
$$

Substituindo $-q^{2}=Q^{2}, x=\frac{Q^{2}}{2 p \cdot q}$ e $p^{2}=m_{p}^{2}$ em (A.3):

$$
\begin{aligned}
T_{1}\left(x, Q^{2}\right) & =\sum_{k=1}^{\infty} \frac{2^{2 k}}{\left(Q^{2}\right)^{2 k}} \sum_{j=0}^{k}(-1)^{j} \frac{(2 k-j) !}{2^{j}(2 k) !} \frac{(2 k) !\left(m_{p}^{2}\right)^{j}\left(-Q^{2}\right)^{j}}{2^{j} j !(2 k-2 j) !}\left(\frac{Q^{2}}{2 x}\right)^{2 k-2 j} C_{1}^{2 k} \mathcal{A}_{2 k} \\
& -\sum_{k=1}^{\infty} \frac{2^{2 k}}{\left(Q^{2}\right)^{2 k}} \sum_{j=1}^{k}(-1)^{j} \frac{(2 k-j) !}{2^{j}(2 k) !} \frac{(2 k-2) !}{2^{j-1}(j-1) !(2 k-2 j) !} \\
& \times\left(m_{p}^{2}\right)^{j}\left(-Q^{2}\right)^{j-1}\left(\frac{Q^{2}}{2 x}\right)^{2 k-2 j} Q^{2} C_{2}^{2 k} \mathcal{A}_{2 k}
\end{aligned}
$$




$$
=\sum_{k=1}^{\infty} \sum_{j=0}^{k} \frac{(2 k-j) !}{j !(2 k-2 j) !}\left(\frac{m_{p}^{2}}{Q^{2}}\right)^{j}\left[C_{1}^{2 k}+\frac{j}{k(2 k-1)} C_{2}^{2 k}\right] \frac{\mathcal{A}_{2 k}}{x^{2 k-2 j}}
$$

Utilizando o binômio $\left(\begin{array}{l}n \\ k\end{array}\right)=\frac{n !}{k !(n-k) !}$ e fazendo a troca, nos índices mudos, $k=l+j$ em (A.4) tem-se:

$$
T_{1}\left(x, Q^{2}\right)=\sum_{l=0}^{\infty} \sum_{j=0}^{\infty}\left(\begin{array}{c}
2 l+j \\
j
\end{array}\right) \mu^{j}\left[C_{1}^{2 l+2 j}+\frac{j}{(l+j)(2 l+2 j-1)} C_{2}^{2 l+2 j}\right] \frac{\mathcal{A}_{2 l+2 j}}{x^{2 l}}
$$

Lembrando que $x=\frac{1}{\omega}$ e usando as equações (2.18), (2.19), (2.35), (3.3) em (A.5) encontramos o momento de Cornwall-Norton (3.18a):

$$
\begin{aligned}
M_{1}^{(n)}\left(Q^{2}\right) & =\int_{0}^{1} x^{n-1} F_{1}^{T M C}\left(x, Q^{2}\right) d x=\frac{1}{2} \frac{1}{2 \pi i} \oint_{C} \frac{1}{\omega^{n+1}} T_{1}\left(1 / \omega, Q^{2}\right) d \omega \\
& =\frac{1}{2} \sum_{l=0}^{\infty} \sum_{j=0}^{\infty}\left(\begin{array}{c}
2 l+j \\
j
\end{array}\right) \mu^{j}\left[C_{1}^{2 l+2 j}+\frac{j}{(l+j)(2 l+2 j-1)} C_{2}^{2 l+2 j}\right] \mathcal{A}_{2 l+2 j} \\
& \times\left(\frac{1}{2 \pi i} \oint_{C} \omega^{2 l-n-1} d \omega\right) \\
& =\frac{1}{2} \sum_{l=0}^{\infty} \sum_{j=0}^{\infty}\left(\begin{array}{c}
2 l+j \\
j
\end{array}\right) \mu^{j}\left[C_{1}^{2 l+2 j}+\frac{j}{(l+j)(2 l+2 j-1)} C_{2}^{2 l+2 j}\right] \mathcal{A}_{2 l+2 j} \delta_{2 l, n} \\
M_{1}^{(n)}\left(Q^{2}\right) & =\sum_{j=0}^{\infty}\left(\begin{array}{c}
n+j \\
j
\end{array}\right) \mu^{j}\left[\frac{C_{1}^{n+2 j}}{2}+\frac{j}{(n+2 j)(n+2 j-1)} C_{2}^{n+2 j}\right] \mathcal{A}_{n+2 j} \quad(\mathrm{~A} .6)
\end{aligned}
$$

Utilizando a transformada inversa de Mellin, (3.19) para $i=1$, (3.20) e (3.22), na equação (A.6), tem-se:

$$
\begin{array}{r}
F_{1}^{T M C}\left(x, Q^{2}\right)=\frac{1}{2 \pi i} \int_{0}^{1} d y \int_{-i \infty}^{+i \infty} d n x^{-n} \sum_{j=0}^{\infty}\left(\begin{array}{c}
n+j \\
j
\end{array}\right) \mu^{j}\left[y^{n+2 j-1} \frac{f_{1}(y)}{2}\right. \\
\left.+j y^{n+2 j-2} g_{2}(y)\right]
\end{array}
$$




$$
\begin{array}{r}
=\frac{1}{2 \pi i} \int_{0}^{1} d y \int_{-i \infty}^{+i \infty} d n x^{-n} y^{n}\left[\sum_{j=0}^{\infty}\left(\begin{array}{c}
n+j \\
j
\end{array}\right)\left(\mu y^{2}\right)^{j} \frac{f_{1}(y)}{2 y}\right. \\
\left.+\sum_{j=1}^{\infty}\left(\begin{array}{c}
n+j \\
j
\end{array}\right)\left(\mu y^{2}\right)^{j} j \frac{g_{2}(y)}{y^{2}}\right]
\end{array}
$$

Para eliminar a soma em $j$ utiliza-se o teorema binomial (3.25) e a relação:

$$
\sum_{j=1}^{\infty}\left(\begin{array}{c}
n+j \\
j
\end{array}\right)\left(\mu y^{2}\right)^{j} j=\sum_{j=1}^{\infty}(n+1)\left(\begin{array}{c}
n+j \\
j-1
\end{array}\right)\left(\mu y^{2}\right)^{j}=\frac{\mu y^{2}(n+1)}{\left(1-\mu y^{2}\right)^{n+2}}
$$

Então (A.7) simplifica e encontramos a equação (3.30a):

$$
\begin{aligned}
& F_{1}^{T M C}\left(x, Q^{2}\right)=\frac{1}{2 \pi i} \int_{0}^{1} d y \int_{-i \infty}^{+i \infty} d n x^{-n} y^{n} \frac{f_{1}(y)}{2 y\left(1-\mu y^{2}\right)^{n+1}} \\
& +\frac{1}{2 \pi i} \int_{0}^{1} d y \int_{-i \infty}^{+i \infty} d n x^{-n} y^{n}(n+1) \frac{\mu g_{2}(y)}{\left(1-\mu y^{2}\right)^{n+2}} \\
& =\left\{\int_{0}^{1} d y \frac{f_{1}(y)}{2 y\left(1-\mu y^{2}\right)}-\mu x^{2} \frac{\partial}{\partial x}\left[\int_{0}^{1} d y \frac{g_{2}(y)}{x\left(1-\mu y^{2}\right)^{2}}\right]\right\} \\
& \times\left[\frac{1}{2 \pi i} \int_{-i \infty}^{+i \infty} d n\left(\frac{y}{x\left(1-\mu y^{2}\right)}\right)^{n}\right] \\
& =\left\{\int_{0}^{1} d y \frac{f_{1}(y)}{2 y\left(1-\mu y^{2}\right)}-\mu x^{2} \frac{\partial}{\partial x}\left[\int_{0}^{1} d y \frac{g_{2}(y)}{x\left(1-\mu y^{2}\right)^{2}}\right]\right\} \\
& \times \delta\left[\ln \left(\frac{y}{x\left(1-\mu y^{2}\right)}\right)\right] \\
& =\left\{\int_{0}^{1} d y \frac{f_{1}(y)}{2 y\left(1-\mu y^{2}\right)}-\mu x^{2} \frac{\partial}{\partial x}\left[\int_{0}^{1} d y \frac{g_{2}(y)}{x\left(1-\mu y^{2}\right)^{2}}\right]\right\} \\
& \times \delta(y-\xi) \frac{y\left(1-\mu y^{2}\right)}{1+\mu y^{2}} \\
& =\frac{f_{1}(\xi)}{2\left(1+\mu \xi^{2}\right)}-\mu x^{2} \frac{\partial}{\partial x}\left[\frac{\xi g_{2}(\xi)}{\left(1-\mu y^{2}\right)\left(1+\mu y^{2}\right)} \frac{\left(1-\mu y^{2}\right)}{\xi}\right]
\end{aligned}
$$




$$
F_{1}^{T M C}\left(x, Q^{2}\right)=\frac{f_{1}(\xi)}{2\left(1+\mu \xi^{2}\right)}-\mu x^{2} \frac{\partial}{\partial x}\left[\frac{g_{2}(\xi)}{1+\mu y^{2}}\right]
$$

onde foi usado que $(n+1) x^{-n}=-x^{2} \frac{\partial}{\partial x} x^{-n-1}$, a propriedade (3.27), a relação (3.28) e $x=\frac{\xi}{\left(1-\mu \xi^{2}\right)}$.

\section{A.2 $\quad F_{L}^{T M C}\left(x, Q^{2}\right)$, Georgi e Politzer}

Para encontrar a $F_{L}^{T M C}\left(x, Q^{2}\right)$ basta usar a relação:

$$
F_{L}^{T M C}\left(x, Q^{2}\right)=\left(1+4 \mu x^{2}\right) F_{2}^{T M C}\left(x, Q^{2}\right)-2 x F_{1}^{T M C}\left(x, Q^{2}\right)
$$

Assim, pode-se encontrar o momento de Cornwall-Norton:

$$
\begin{aligned}
M_{L}^{(n)}\left(Q^{2}\right) & =\int_{0}^{1} d x x^{n-2} F_{L}^{T M C}\left(x, Q^{2}\right) \\
& =\int_{0}^{1} d x x^{n-2} F_{2}^{T M C}\left(x, Q^{2}\right)+4 \mu \int_{0}^{1} d x x^{(n+2)-2} F_{2}^{T M C}\left(x, Q^{2}\right) \\
& -2 \int_{0}^{1} d x x^{n-1} F_{1}^{T M C}\left(x, Q^{2}\right) \\
& \left.=M_{2}^{(n)}\left(Q^{2}\right)+4 \mu M_{2}^{(n+2)}\left(Q^{2}\right)-2 M_{1}^{(n)}\left(Q^{2}\right)\right) \\
& =\sum_{j=0}^{\infty}\left(\begin{array}{c}
n+j \\
j
\end{array}\right) \mu^{j}\left[-C_{1}^{n+2 j}+\frac{n(n-1)-2 j}{(n+2 j)(n+2 j-1)} C_{2}^{n+2 j}\right] \mathcal{A}_{n+2 j} \\
& +4 \sum_{j=0}^{\infty}\left(\begin{array}{c}
n+j+2 \\
j
\end{array}\right) \mu^{j+1} \frac{(n+2)(n+1) C_{2}^{n+2 j+2}}{(n+2 j+2)(n+2 j+1)} \mathcal{A}_{n+2 j+2}
\end{aligned}
$$

onde foi usado as equações (3.17) e (3.18a). Fazendo a mudança $j+1 \rightarrow j$ nos índices 
mudos do segundo termo de (A.11), chega-se:

$$
\begin{aligned}
M_{L}^{(n)}\left(Q^{2}\right)=\sum_{j=0}^{\infty}\left(\begin{array}{c}
n+j \\
j
\end{array}\right) \mu^{j}\left[-C_{1}^{n+2 j}\right. & \\
& \left.+\frac{n(n-1)-2 j+4 j(n+j+1)}{(n+2 j)(n+2 j-1)} C_{2}^{n+2 j}\right] \mathcal{A}_{n+2 j}
\end{aligned}
$$

como $C_{L}^{n+2 j}=C_{2}^{n+2 j}-C_{1}^{n+2 j}$, chegamos na equação (3.18b):

$$
M_{L}^{(n)}\left(Q^{2}\right)=\sum_{j=0}^{\infty}\left(\begin{array}{c}
n+j \\
j
\end{array}\right) \mu^{j}\left[C_{L}^{n+2 j}+\frac{4 j}{(n+2 j)(n+2 j-1)} C_{2}^{n+2 j}\right] \mathcal{A}_{n+2 j}
$$

Utilizando as equações (3.29) e (A.9) em (A.10), encontra-se a equação (3.30b):

$$
\begin{aligned}
F_{L}^{T M C}\left(x, Q^{2}\right)= & \left(1+4 \mu x^{2}\right) x^{2} \frac{\partial^{2}}{\partial x^{2}}\left[\frac{x g_{2}(\xi)}{\xi\left(1+\mu \xi^{2}\right)}\right] \\
- & 2 x\left\{\frac{f_{1}(\xi)}{2\left(1+\mu \xi^{2}\right)}-\mu x^{2} \frac{\partial}{\partial x}\left[\frac{g_{2}(\xi)}{1+\mu y^{2}}\right]\right\} \\
F_{L}^{T M C}\left(x, Q^{2}\right)= & -\frac{x f_{1}(\xi)}{1+\mu \xi^{2}}+2 \mu x^{3} \frac{\partial}{\partial x}\left[\frac{g_{2}(\xi)}{1+\mu y^{2}}\right] \\
& +\left(1+4 \mu x^{2}\right) x^{2} \frac{\partial^{2}}{\partial x^{2}}\left[\frac{x g_{2}(\xi)}{\xi\left(1+\mu \xi^{2}\right)}\right]
\end{aligned}
$$

\section{A.3 $F_{3}^{T M C}\left(x, Q^{2}\right)$, Georgi e Politzer}

Primeiro é preciso encontrar $\frac{T_{3}}{m_{p}^{2}}$, que é o coeficiente de $-i \epsilon^{\mu \nu \lambda \rho} p_{\lambda} q_{\rho}$ em (3.8), que terá contribuição parcial do termo $C_{3}^{2 k}$, pois $\left(-i \epsilon^{\mu \nu \lambda \rho} g_{\lambda \mu_{1}} q_{\rho} q_{\mu_{2}} C_{3}^{2 k}\right)\left(p^{\mu_{1}} p^{\mu_{2}}\right)=$ $-i \epsilon^{\mu \nu \lambda \rho} p_{\lambda} q_{\rho} p \cdot q C_{3}^{2 k}$.

Assim, fixando $k \in \mathcal{N}$ e $j \in\{0, \ldots, k\}$, utilizando os termos de $\{g \ldots g\}\{p \ldots p\}$ que contenham $p^{\mu_{i}}$ e distribuindo os índices $\mu_{2}, \ldots, \mu_{2 k}$ pelos $j g^{\mu_{i} \mu_{l}}$ e $(2 k-2 j-1) p^{\mu_{i}}$ (sem criar produtos duplicados) precisa-se subtrair este $p^{\mu_{1}}$, que foi contraído da soma 
simétrica:

$$
\begin{aligned}
& \sum_{k=1}^{\infty}\left(-i \epsilon^{\mu \nu \lambda \rho} g_{\lambda \mu_{1}} q_{\rho} q_{\mu_{2}} C_{3}^{2 k}\right) q_{\mu_{3}} \ldots q_{\mu_{2 k}} \frac{2^{2 k}}{\left(Q^{2}\right)^{2 k}} \mathcal{A}_{2 k} \Pi^{\mu_{1} \ldots \mu_{2 k}}= \\
& =\quad-i \epsilon^{\mu \nu \lambda \rho} p_{\lambda} q_{\rho} \sum_{k=1}^{\infty} \frac{2^{2 k}}{\left(Q^{2}\right)^{2 k}} \sum_{j=0}^{k-1}(-1)^{j} \frac{(2 k-j) !}{2^{j}(2 k) !} \frac{(2 k-1) !}{2^{j} j !(2 k-2 j-1) !}\left(p^{2} q^{2}\right)^{j} \\
& \times \quad(p \cdot q)^{2 k-2 j-1} C_{3}^{2 k} \mathcal{A}_{2 k}+\text { termos sem } p^{\mu_{i}}
\end{aligned}
$$

Então:

$$
\begin{aligned}
\frac{T_{3}\left(x, Q^{2}\right)}{m_{p}^{2}}=\sum_{k=1}^{\infty} \frac{2^{2 k}}{\left(Q^{2}\right)^{2 k}} \sum_{j=0}^{k-1}(-1)^{j} \frac{(2 k-j) !}{2^{j}(2 k) !} \frac{(2 k-1) !}{2^{j} j !(2 k-2 j-1) !}\left(p^{2} q^{2}\right)^{j} \\
\times(p \cdot q)^{2 k-2 j-1} C_{3}^{2 k} \mathcal{A}_{2 k}
\end{aligned}
$$

Substituindo $-q^{2}=Q^{2}, x=\frac{Q^{2}}{2 p \cdot q}$ e $p^{2}=m_{p}^{2}$ em (A.16):

$$
\begin{aligned}
\frac{T_{3}\left(x, Q^{2}\right)}{m_{p}^{2}}= & \sum_{k=1}^{\infty} \frac{2^{2 k}}{\left(Q^{2}\right)^{2 k}} \sum_{j=0}^{k-1}(-1)^{j} \frac{(2 k-j) !}{2^{j}(2 k) !} \frac{(2 k-1) !}{2^{j} j !(2 k-2 j-1) !} \\
& \times\left(m_{p}^{2}\right)^{j}\left(-Q^{2}\right)^{j}\left(\frac{Q^{2}}{2 x}\right)^{2 k-2 j-1} C_{3}^{2 k} \mathcal{A}_{2 k} \\
= & \frac{2}{Q^{2}} \sum_{k=1}^{\infty} \sum_{j=0}^{k-1} \frac{(2 k-j) !}{j !(2 k-2 j) !} \frac{(2 k-2 j) !}{(2 k-2 j-1) !} \frac{(2 k-1) !}{(2 k) !}\left(\frac{m_{p}^{2}}{Q^{2}}\right)^{j} C_{3}^{2 k} \frac{\mathcal{A}_{2 k}}{x^{2 k-2 j-1}} \\
= & \frac{2}{Q^{2}} \sum_{k=1}^{\infty} \sum_{j=0}^{k-1} \frac{(2 k-j) !}{j !(2 k-2 j) !} \frac{(k-j)}{k}\left(\frac{m_{p}^{2}}{Q^{2}}\right)^{j} C_{3}^{2 k} \frac{\mathcal{A}_{2 k}}{x^{2 k-2 j-1}}
\end{aligned}
$$

Utilizando o binômio $\left(\begin{array}{l}n \\ k\end{array}\right)=\frac{n !}{k !(n-k) !}$ e fazendo a troca, nos índices mudos, $k=l+j$ em (A.17) tem-se:

$$
T_{3}\left(x, Q^{2}\right)=2 \sum_{l=1}^{\infty} \sum_{j=0}^{\infty}\left(\begin{array}{c}
2 l+j \\
j
\end{array}\right) \mu^{j+1} \frac{l}{(l+j)} \frac{C_{3}^{2 l+2 j} \mathcal{A}_{2 l+2 j}}{x^{2 l-1}}
$$


Lembrando que $x=\frac{1}{\omega}$ e usando as equações (2.18), (2.19), (2.35), (3.3) em (A.18) encontramos o momento de Cornwall-Norton (3.18c):

$$
\begin{aligned}
M_{3}^{(n)}\left(Q^{2}\right) & =\int_{0}^{1} x^{n-1} F_{3}^{T M C}\left(x, Q^{2}\right) d x=\frac{1}{2} \frac{1}{2 \pi i} \oint_{C} \frac{1}{\omega^{n+1}} \frac{\omega}{\mu} T_{3}\left(1 / \omega, Q^{2}\right) d \omega \\
& =\sum_{l=1}^{\infty} \sum_{j=0}^{\infty}\left(\begin{array}{c}
2 l+j \\
j
\end{array}\right) \mu^{j} \frac{l}{(l+j)} C_{3}^{2 l+2 j} \mathcal{A}_{2 l+2 j}\left(\frac{1}{2 \pi i} \oint_{C} \omega^{2 l-n-1} d \omega\right) \\
& =\sum_{l=1}^{\infty} \sum_{j=0}^{\infty}\left(\begin{array}{c}
2 l+j \\
j
\end{array}\right) \mu^{j} \frac{l}{(l+j)} C_{3}^{2 l+2 j} \mathcal{A}_{2 l+2 j} \delta_{2 l, n} \\
M_{3}^{(n)}\left(Q^{2}\right) & =\sum_{j=0}^{\infty}\left(\begin{array}{c}
n+j \\
j
\end{array}\right) \mu^{j} \frac{n}{(n+2 j)} C_{3}^{n+2 j} \mathcal{A}_{n+2 j}
\end{aligned}
$$

Utilizando a transformada inversa de Mellin, (3.19) para $i=3$ e a substituição:

$$
\frac{C_{3}^{n+2 j} \mathcal{A}_{n+2 j}}{(n+2 j)}=\int_{0}^{1} d y y^{n+2 j-1} h_{3}(y)
$$

a equação (A.19) simplifica e com um pouco de álgebra chegamos em (3.30c):

$$
\begin{aligned}
F_{3}^{T M C}\left(x, Q^{2}\right) & =\frac{1}{2 \pi i} \int_{0}^{1} d y \int_{-i \infty}^{+i \infty} d n x^{-n} \sum_{j=0}^{\infty}\left(\begin{array}{c}
n+j \\
j
\end{array}\right) \mu^{j} n y^{n+2 j-1} h_{3}(y) \\
& =-x \frac{\partial}{\partial x}\left\{\int_{0}^{1} d y \frac{h_{3}(y)}{y\left(1-\mu y^{2}\right)}\left[\frac{1}{2 \pi i} \int_{-i \infty}^{+i \infty} d n\left(\frac{y}{x\left(1-\mu y^{2}\right)}\right)^{n}\right]\right\} \\
& =-x \frac{\partial}{\partial x}\left\{\int_{0}^{1} d y \frac{h_{3}(y)}{y\left(1-\mu y^{2}\right)} \delta\left[\ln \left(\frac{y}{x\left(1-\mu y^{2}\right)}\right)\right]\right\} \\
& =-x \frac{\partial}{\partial x}\left\{\int_{0}^{1} d y \frac{h_{3}(y)}{y\left(1-\mu y^{2}\right)} \delta(y-\xi) \frac{y\left(1-\mu y^{2}\right)}{1+\mu y^{2}}\right\} \\
F_{3}^{T M C}\left(x, Q^{2}\right) & =-x \frac{\partial}{\partial x}\left[\frac{h_{3}(\xi)}{1+\mu \xi^{2}}\right]
\end{aligned}
$$


onde foi usado que $n x^{-n}=-x \frac{\partial}{\partial x} x^{-n}$, o teorema binomial (3.25), a propriedade (3.27) e a relação (3.28).

\section{A.4 $F_{4}^{T M C}\left(x, Q^{2}\right)$, Georgi e Politzer}

Primeiro é preciso encontrar $\frac{T_{4}}{m_{p}^{2}}$, que é o coeficiente de $q^{\mu} q^{\nu}$ em (3.8), que terá contribuição parcial do termo $C_{2}^{2 k}$, pois $\left(g_{\mu_{1}}^{\mu} g_{\mu_{2}}^{\nu} Q^{2} C_{2}^{2 k}\right)\left(g^{\mu_{1} \mu_{i}} g^{\mu_{2} \mu_{l}}\right)=q^{\mu} q^{\nu} Q^{2} C_{2}^{2 k}$, integral de $C_{4}^{2 k}$ e parcial de $C_{5}^{2 k}$, através de $\left[\left(g_{\mu_{1}}^{\mu} q^{\nu} q_{\mu_{2}}+g_{\mu_{1}}^{\nu} q^{\mu} q_{\mu_{2}}\right) C_{5}^{2 k}\right]\left(g^{\mu_{1} \mu_{2}}\right)=2 q^{\mu} q^{\nu} C_{5}^{2 k}$.

Para $C_{2}^{2 k}$, fixando $k \in \mathcal{N}$ e $j \in\{2, \ldots, k\}$, com os termos de $\{g \ldots g\}\{p \ldots p\}$ que contenham $g^{\mu_{1} \mu_{i}} g^{\mu_{2} \mu_{l}}$ para $i, l \in\{3, \ldots, 2 k\}, i \neq l$ e distribuindo os índices restantes $\mu_{3}, \ldots, \mu_{2 k}$ pelos $(j-2) g^{\mu_{i} \mu_{l}}$ e $(2 k-2 j) p^{\mu_{i}}$ (sem criar produtos duplicados) precisa-se subtrair os $g^{\mu_{1} \mu_{i}} g^{\mu_{2} \mu_{l}}$, que foram contraídos da soma simétrica:

$$
\begin{aligned}
& \sum_{k=1}^{\infty}\left(g_{\mu_{1}}^{\mu} g_{\mu_{2}}^{\nu} Q^{2} C_{2}^{2 k}\right) q_{\mu_{3}} \ldots q_{\mu_{2 k}} \frac{2^{2 k}}{\left(Q^{2}\right)^{2 k}} \mathcal{A}_{2 k} \Pi^{\mu_{1} \ldots \mu_{2 k}}= \\
& =\quad q^{\mu} q^{\nu} Q^{2} \sum_{k=1}^{\infty} \frac{2^{2 k}}{\left(Q^{2}\right)^{2 k}} \sum_{j=2}^{k}(-1)^{j} \frac{(2 k-j) !}{2^{j}(2 k) !} \frac{(2 k-2) !}{2^{j-2}(j-2) !(2 k-2 j) !}\left(p^{2}\right)^{j}\left(q^{2}\right)^{j-2} \\
& \times \quad(p \cdot q)^{2 k-2 j} C_{2}^{2 k} \mathcal{A}_{2 k}+\text { termos sem } g^{\mu_{n} \mu_{m}}
\end{aligned}
$$

Para $C_{4}^{2 k}$, fixando $k \in \mathcal{N}$ e $j \in\{0, \ldots, k\}$, com cada termo de $\{g \ldots g\}\{p \ldots p\}$, encontra-se que $j q_{\mu_{i}}$ terão seus índices levantados por $j g^{\mu_{i} \mu_{l}}$, que vão se contrair com $j q_{\mu_{l}}$ resultando em $\left(q^{2}\right)^{j}$. Os $(2 k-2 j) q_{\mu_{i}}$ irão contrair com os $(2 k-2 j) p^{\mu_{i}}$ dando $(p \cdot q)^{2 k-2 j}$ :

$$
\begin{aligned}
& \sum_{k=1}^{\infty}\left(\frac{q^{\mu} q^{\nu}}{Q^{2}} q_{\mu_{1}} q_{\mu_{2}} C_{4}^{2 k}\right) q_{\mu_{3}} \ldots q_{\mu_{2 k}} \frac{2^{2 k}}{\left(Q^{2}\right)^{2 k}} \mathcal{A}_{2 k} \Pi^{\mu_{1} \ldots \mu_{2 k}}= \\
& =\frac{q^{\mu} q^{\nu}}{Q^{2}} \sum_{k=1}^{\infty} \frac{2^{2 k}}{\left(Q^{2}\right)^{2 k}} \sum_{j=0}^{k}(-1)^{j} \frac{(2 k-j) !}{2^{j}(2 k) !} \frac{(2 k) !}{2^{j} j !(2 k-2 j) !}\left(p^{2} q^{2}\right)^{j} \\
& \times \quad(p \cdot q)^{2 k-2 j} C_{4}^{2 k} \mathcal{A}_{2 k}
\end{aligned}
$$


Para $C_{5}^{2 k}$, fixando $k \in \mathcal{N}$ e $j \in\{1, \ldots, k\}$, com cada termo de $\{g \ldots g\}\{p \ldots p\}$ que contenham $g^{\mu_{1} \mu_{2}}$ e distribuindo os índices restantes $\mu_{3}, \ldots, \mu_{2 k}$ pelos $(j-1) g^{\mu_{i} \mu_{l}}$ e $(2 k-2 j) p^{\mu_{i}}$ (sem criar produtos duplicados) precisa-se fazer $j \rightarrow j-1$ e $k \rightarrow k-1$ :

$$
\begin{aligned}
& \sum_{k=1}^{\infty}\left(\left(g_{\mu_{1}}^{\mu} q^{\nu} q_{\mu_{2}}+g_{\mu_{1}}^{\nu} q^{\mu} q_{\mu_{2}}\right) C_{5}^{2 k}\right) q_{\mu_{3}} \ldots q_{\mu_{2 k}} \frac{2^{2 k}}{\left(Q^{2}\right)^{2 k}} \mathcal{A}_{2 k} \Pi^{\mu_{1} \ldots \mu_{2 k}}= \\
& =2 q^{\mu} q^{\nu} \sum_{k=1}^{\infty} \frac{2^{2 k}}{\left(Q^{2}\right)^{2 k}} \sum_{j=1}^{k}(-1)^{j} \frac{(2 k-j) !}{2^{j}(2 k) !} \frac{(2 k-2) !}{2^{j-1}(j-1) !(2 k-2 j) !}\left(p^{2}\right)^{j}\left(q^{2}\right)^{j-1} \\
& \times \quad(p \cdot q)^{2 k-2 j} C_{5}^{2 k} \mathcal{A}_{2 k}+\text { termos sem } g^{\mu_{n} \mu_{m}}
\end{aligned}
$$

Então com (A.22), (A.23) e (A.24):

$$
\begin{aligned}
\frac{T_{4}\left(x, Q^{2}\right)}{m_{p}^{2}} & =Q^{2} \sum_{k=1}^{\infty} \frac{2^{2 k}}{\left(Q^{2}\right)^{2 k}} \sum_{j=2}^{k}(-1)^{j} \frac{(2 k-j) !}{2^{j}(2 k) !} \frac{(2 k-2) !}{2^{j-2}(j-2) !(2 k-2 j) !}\left(p^{2}\right)^{j}\left(q^{2}\right)^{j-2} \\
& \times(p \cdot q)^{2 k-2 j} C_{2}^{2 k} \mathcal{A}_{2 k} \\
& +\frac{1}{Q^{2}} \sum_{k=1}^{\infty} \frac{2^{2 k}}{\left(Q^{2}\right)^{2 k}} \sum_{j=0}^{k}(-1)^{j} \frac{(2 k-j) !}{2^{j}(2 k) !} \frac{(2 k) !}{2^{j} j !(2 k-2 j) !}\left(p^{2} q^{2}\right)^{j} \\
& \times(p \cdot q)^{2 k-2 j} C_{4}^{2 k} \mathcal{A}_{2 k} \\
& +2 \sum_{k=1}^{\infty} \frac{2^{2 k}}{\left(Q^{2}\right)^{2 k}} \sum_{j=1}^{k}(-1)^{j} \frac{(2 k-j) !}{2^{j}(2 k) !} \frac{(2 k-2) !}{2^{j-1}(j-1) !(2 k-2 j) !}\left(p^{2}\right)^{j}\left(q^{2}\right)^{j-1} \\
& \times(p \cdot q)^{2 k-2 j} C_{5}^{2 k} \mathcal{A}_{2 k}
\end{aligned}
$$

Substituindo $-q^{2}=Q^{2}, x=\frac{Q^{2}}{2 p \cdot q}$ e $p^{2}=m_{p}^{2}$ em (A.25):

$$
\begin{aligned}
\frac{T_{4}\left(x, Q^{2}\right)}{m_{p}^{2}} & =Q^{2} \sum_{k=1}^{\infty} \frac{2^{2 k}}{\left(Q^{2}\right)^{2 k}} \sum_{j=2}^{k}(-1)^{j} \frac{(2 k-j) !}{2^{j}(2 k) !} \frac{(2 k-2) !}{2^{j-2}(j-2) !(2 k-2 j) !}\left(m_{p}^{2}\right)^{j}\left(-Q^{2}\right)^{j-2} \\
& \times\left(\frac{Q^{2}}{2 x}\right)^{2 k-2 j} C_{2}^{2 k} \mathcal{A}_{2 k} \\
& +\frac{1}{Q^{2}} \sum_{k=1}^{\infty} \frac{2^{2 k}}{\left(Q^{2}\right)^{2 k}} \sum_{j=0}^{k}(-1)^{j} \frac{(2 k-j) !}{2^{j}(2 k) !} \frac{(2 k) !}{2^{j} j !(2 k-2 j) !}\left(m_{p}^{2}\right)^{j}\left(-Q^{2}\right)^{j} \\
& \times\left(\frac{Q^{2}}{2 x}\right)^{2 k-2 j} C_{4}^{2 k} \mathcal{A}_{2 k}
\end{aligned}
$$




$$
\begin{gathered}
+2 \sum_{k=1}^{\infty} \frac{2^{2 k}}{\left(Q^{2}\right)^{2 k}} \sum_{j=1}^{k}(-1)^{j} \frac{(2 k-j) !}{2^{j}(2 k) !} \frac{(2 k-2) !}{2^{j-1}(j-1) !(2 k-2 j) !}\left(m_{p}^{2}\right)^{j}\left(-Q^{2}\right)^{j-1} \\
\times\left(\frac{Q^{2}}{2 x}\right)^{2 k-2 j} C_{5}^{2 k} \mathcal{A}_{2 k} \\
=\sum_{k=1}^{\infty} \sum_{j=0}^{k} \frac{(2 k-j) !}{j !(2 k-2 j) !}\left(\frac{m_{p}^{2}}{Q^{2}}\right)^{j} \frac{1}{Q^{2}}\left[\frac{2 j(j-1)}{k(2 k-1)} C_{2}^{2 k}+C_{4}^{2 k}\right. \\
\left.\quad-\frac{2 j}{k(2 k-1)} C_{5}^{2 k}\right] \frac{\mathcal{A}_{2 k}}{x^{2 k-2 j}}
\end{gathered}
$$

Utilizando o binômio $\left(\begin{array}{l}n \\ k\end{array}\right)=\frac{n !}{k !(n-k) !}$ e fazendo a troca, nos índices mudos, $k=l+j$ em (A.26) tem-se:

$$
\begin{array}{r}
T_{4}\left(x, Q^{2}\right)=\sum_{l=0}^{\infty} \sum_{j=0}^{\infty}\left(\begin{array}{c}
2 l+j \\
j
\end{array}\right) \mu^{j+1}\left[\frac{2 j(j-1)}{(l+j)(2 l+2 j-1)} C_{2}^{2 l+2 j}+C_{4}^{2 l+2 j}\right. \\
\left.-\frac{2 j}{(l+j)(2 l+2 j-1)} C_{5}^{2 l+2 j}\right] \frac{\mathcal{A}_{2 l+2 j}}{x^{2 l}}
\end{array}
$$

Lembrando que $x=\frac{1}{\omega}$ e usando as equações (2.18), (2.19), (2.35), (3.3) em (A.27) encontramos o momento de Cornwall-Norton (3.18d):

$$
\begin{aligned}
M_{4}^{(n)}\left(Q^{2}\right)= & \int_{0}^{1} x^{n-1} F_{4}^{T M C}\left(x, Q^{2}\right) d x=\frac{1}{2} \frac{1}{2 \pi i} \oint_{C} \frac{1}{\omega^{n+1}} \frac{1}{2 \mu} T_{4}\left(1 / \omega, Q^{2}\right) d \omega \\
= & \sum_{l=1}^{\infty} \sum_{j=0}^{\infty}\left(\begin{array}{c}
2 l+j \\
j
\end{array}\right) \mu^{j}\left[\frac{j(j-1)}{2(l+j)(2 l+2 j-1)} C_{2}^{2 l+2 j}+\frac{C_{4}^{2 l+2 j}}{4}\right. \\
= & \sum_{l=1}^{\infty} \sum_{j=0}^{\infty}\left(\begin{array}{c}
2 l+j \\
j
\end{array}\right) \mu^{j}\left[\frac{j}{2(l+j)(2 l+2 j-1)} C_{5}^{2 l+2 j}\right] \mathcal{A}_{2 l+2 j}\left(\oint_{C} \omega^{2 l-n-1} d \omega\right) \\
& -\frac{j(j-1)}{2(l+j)(2 l+2 j-1)} C_{2}^{2 l+2 j}+\frac{C_{4}^{2 l+2 j}}{4}
\end{aligned}
$$




$$
\begin{array}{r}
M_{4}^{(n)}\left(Q^{2}\right)=\sum_{j=0}^{\infty}\left(\begin{array}{c}
n+j \\
j
\end{array}\right) \mu^{j}\left[\frac{j(j-1)}{(n+2 j)(n+2 j-1)} C_{2}^{n+2 j}+\frac{C_{4}^{n+2 j}}{4}\right. \\
\left.-\frac{j}{(n+2 j)(n+2 j-1)} C_{5}^{n+2 j}\right] \mathcal{A}_{n+2 j}
\end{array}
$$

Utilizando a transformada inversa de Mellin, (3.19) para $i=4,(3.20),(3.22)$ e a substituição:

$$
\frac{C_{5}^{n+2 j} \mathcal{A}_{n+2 j}}{(n+2 j)(n+2 j-1)}=\int_{0}^{1} d y y^{n+2 j-2} g_{5}(y)
$$

a equação (A.28) simplifica e com um pouco de álgebra chegamos em (3.30d):

$$
\begin{aligned}
F_{4}^{T M C}\left(x, Q^{2}\right)= & \frac{1}{2 \pi i} \int_{0}^{1} d y \int_{-i \infty}^{+i \infty} d n x^{-n} \sum_{j=0}^{\infty}\left(\begin{array}{c}
n+j \\
j
\end{array}\right) \mu^{j}\left[y^{n+2 j-2} j(j-1) g_{2}(y)\right. \\
= & \left\{\mu^{2} x^{3} \frac{\partial^{2}}{\partial x^{2}}\left[\int_{0}^{1} d y \frac{y^{2} g_{2}(y)}{x\left(1-\mu y^{2}\right)^{3}}\right]+\int_{0}^{1} d y \frac{f_{4}(y)}{4 y\left(1-\mu y^{2}\right)}\right. \\
& \left.+\mu x^{2} \frac{\partial}{\partial x}\left[\int_{0}^{1} d y \frac{g_{5}(y)}{x\left(1-\mu y^{2}\right)^{2}}\right]\right\}\left[\frac{1}{2 \pi i} \int_{-i \infty}^{+i \infty} d n\left(\frac{y}{x\left(1-\mu y^{2}\right)}\right)^{n}\right] \\
= & \left\{\mu^{2} x^{3} \frac{\partial^{2}}{\partial x^{2}}\left[\int_{0}^{1} d y \frac{y^{2} g_{2}(y)}{x\left(1-\mu y^{2}\right)^{3}}\right]+\int_{0}^{1} d y \frac{f_{4}(y)}{4 y\left(1-\mu y^{2}\right)}\right. \\
& \left.\left.+\mu x^{2} \frac{\partial}{\partial x}\left[\int_{0}^{1} d y \frac{g_{5}(y)}{x\left(1-\mu y^{2}\right)^{2}}\right]\right\} \delta \ln \left(\frac{y}{x\left(1-\mu y^{2}\right)}\right)\right] \\
= & \left\{\mu^{2} x^{3} \frac{\partial^{2}}{\partial x^{2}}\left[\int_{0}^{1} d y \frac{y^{2} g_{2}(y)}{\left(1-\mu y^{2}\right)^{3}} \frac{\left(1-\mu y^{2}\right)}{\xi}\right]+\int_{0}^{1} d y \frac{f_{4}(y)}{4 y\left(1-\mu y^{2}\right)}\right. \\
& +\mu x^{2} \frac{\partial}{\partial x}\left[\int_{0}^{1} d y \frac{g_{5}(y)}{\left(1-\mu y^{2}\right)^{2}} \frac{\left(1-\mu y^{2}\right)}{\xi}\right] \delta(y-\xi) \frac{y\left(1-\mu y^{2}\right)}{1+\mu y^{2}} \\
F_{4}^{T M C}\left(x, Q^{2}\right)= & \mu^{2} x^{3} \frac{\partial^{2}}{\partial x^{2}}\left[\frac{\xi^{2} g_{2}(\xi)}{1-\mu^{2} \xi^{4}}\right]+\frac{f_{4}(\xi)}{4\left(1+\mu \xi^{2}\right)}+\mu x^{2} \frac{\partial}{\partial x}\left[\frac{g_{5}(\xi)}{1+\mu \xi^{2}}\right] \quad(\mathrm{A} .30)
\end{aligned}
$$


onde foi usado que $(n+2)(n+1) x^{-n}=x^{3} \frac{\partial^{2}}{\partial x^{2}} x^{-n-1},(n+1) x^{-n}=-x^{2} \frac{\partial}{\partial x} x^{-n-1}$, o teorema binomial (3.25), a relação (A.8), a propriedade (3.27), a relação (3.28), $x=\frac{\xi}{\left(1-\mu \xi^{2}\right)} \mathrm{e}:$

$$
\sum_{j=1}^{\infty}\left(\begin{array}{c}
n+j \\
j
\end{array}\right)\left(\mu y^{2}\right)^{j} j(j-1)=\left(\mu y^{2}\right)^{2} \frac{(n+2)(n+1)}{\left(1-\mu y^{2}\right)^{n+3}}
$$

\section{A.5 $F_{5}^{T M C}\left(x, Q^{2}\right)$, Georgi e Politzer}

Primeiro é preciso encontrar $\frac{T_{5}}{m_{p}^{2}}$, que é o coeficiente de $\left(p^{\mu} q^{\nu}+p^{\nu} q^{\mu}\right)$ em (3.8), que terá contribuição parcial do termo $C_{2}^{2 k}$, pois $\left(g_{\mu_{1}}^{\mu} g_{\mu_{2}}^{\nu} Q^{2} C_{2}^{2 k}\right)\left(p^{\mu_{1}} q_{\mu_{i}} g^{\mu_{2} \mu_{i}}\right)=p^{\mu} q^{\nu} Q^{2} C_{2}^{2 k}$ e $\left(g_{\mu_{1}}^{\mu} g_{\mu_{2}}^{\nu} Q^{2} C_{2}^{2 k}\right)\left(p^{\mu_{2}} q_{\mu_{l}} g^{\mu_{1} \mu_{l}}\right)=p^{\nu} q^{\mu} Q^{2} C_{2}^{2 k}$ e também parcial de $C_{5}^{2 k}$, através de $\left[\left(g_{\mu_{1}}^{\mu} q^{\nu} q_{\mu_{2}}+g_{\mu_{1}}^{\nu} q^{\mu} q_{\mu_{2}}\right) C_{5}^{2 k}\right]\left(p^{\mu_{1}}\right)=\left(p^{\mu} q^{\nu}+p^{\nu} q^{\mu}\right) C_{5}^{2 k}$.

Para $C_{2}^{2 k}$, fixando $k \in \mathcal{N}$ e $j \in\{2, \ldots, k-1\}$, com os termos de $\{g \ldots g\}\{p \ldots p\}$ que contenham $p^{\mu_{1}} q_{\mu_{i}} g^{\mu_{2} \mu_{i}}$ e distribuindo os índices restantes $\mu_{3}, \ldots, \mu_{2 k}$ pelos $(j-1) g^{\mu_{i} \mu_{l}}$ e $(2 k-2 j-1) p^{\mu_{i}}$ (sem criar produtos duplicados) precisa-se subtrair o $g^{\mu_{i} \mu_{l}}$ e $p^{\mu_{i}}$, que foram contraídos da soma simétrica:

$$
\begin{aligned}
& \sum_{k=1}^{\infty}\left(g_{\mu_{1}}^{\mu} g_{\mu_{2}}^{\nu} Q^{2} C_{2}^{2 k}\right) q_{\mu_{3}} \ldots q_{\mu_{2 k}} \frac{2^{2 k}}{\left(Q^{2}\right)^{2 k}} \mathcal{A}_{2 k} \Pi^{\mu_{1} \ldots \mu_{2 k}}= \\
& =\quad\left(p^{\mu} q^{\nu}+p^{\nu} q^{\mu}\right) Q^{2} \sum_{k=1}^{\infty} \frac{2^{2 k}}{\left(Q^{2}\right)^{2 k}} \sum_{j=1}^{k-1}(-1)^{j} \frac{(2 k-j) !}{2^{j}(2 k) !} \frac{(2 k-2) !}{2^{j-1}(j-1) !(2 k-2 j-1) !} \\
& \times \quad\left(p^{2}\right)^{j}\left(q^{2}\right)^{j-1}(p \cdot q)^{2 k-2 j-1} C_{2}^{2 k} \mathcal{A}_{2 k}+\text { termos com somente } g^{\mu_{n} \mu_{m}} \\
& +\quad \text { termos com somente } p^{\mu_{m}}
\end{aligned}
$$

Para $C_{5}^{2 k}$, fixando $k \in \mathcal{N}$ e $j \in\{1, \ldots, k-1\}$, com cada termo de $\{g \ldots g\}\{p \ldots p\}$ que contenham $p^{\mu_{1}}$ e distribuindo os índices restantes $\mu_{2}, \ldots, \mu_{2 k}$ pelos $j g^{\mu_{i} \mu_{l}}$ e $(2 k-$ $2 j-1) p^{\mu_{i}}$ (sem criar produtos duplicados) precisa-se fazer subtrair os termos utilizados 
da soma simétrica:

$$
\begin{aligned}
& \sum_{k=1}^{\infty}\left(\left(g_{\mu_{1}}^{\mu} q^{\nu} q_{\mu_{2}}+g_{\mu_{1}}^{\nu} q^{\mu} q_{\mu_{2}}\right) C_{5}^{2 k}\right) q_{\mu_{3}} \ldots q_{\mu_{2 k}} \frac{2^{2 k}}{\left(Q^{2}\right)^{2 k}} \mathcal{A}_{2 k} \Pi^{\mu_{1} \ldots \mu_{2 k}}= \\
& =\quad\left(p^{\mu} q^{\nu}+p^{\nu} q^{\mu}\right) \sum_{k=1}^{\infty} \frac{2^{2 k}}{\left(Q^{2}\right)^{2 k}} \sum_{j=1}^{k-1}(-1)^{j} \frac{(2 k-j) !}{2^{j}(2 k) !} \frac{(2 k-1) !}{2^{j} j !(2 k-2 j-1) !}\left(p^{2} q^{2}\right)^{j} \\
& \times \quad(p \cdot q)^{2 k-2 j-1} C_{5}^{2 k} \mathcal{A}_{2 k}+\text { termos com somente } g^{\mu_{n} \mu_{m}}
\end{aligned}
$$

Então com (A.32) e (A.33):

$$
\begin{aligned}
\frac{T_{5}\left(x, Q^{2}\right)}{m_{p}^{2}} & =Q^{2} \sum_{k=1}^{\infty} \frac{2^{2 k}}{\left(Q^{2}\right)^{2 k}} \sum_{j=1}^{k-1}(-1)^{j} \frac{(2 k-j) !}{2^{j}(2 k) !} \frac{(2 k-2) !}{2^{j-1}(j-1) !(2 k-2 j-1) !}\left(p^{2}\right)^{j}\left(q^{2}\right)^{j-1} \\
& \times(p \cdot q)^{2 k-2 j-1} C_{2}^{2 k} \mathcal{A}_{2 k} \\
& +\sum_{k=1}^{\infty} \frac{2^{2 k}}{\left(Q^{2}\right)^{2 k}} \sum_{j=0}^{k-1}(-1)^{j} \frac{(2 k-j) !}{2^{j}(2 k) !} \frac{(2 k-1) !}{2^{j} j !(2 k-2 j-1) !}\left(p^{2} q^{2}\right)^{j} \\
& \times(p \cdot q)^{2 k-2 j-1} C_{5}^{2 k} \mathcal{A}_{2 k}
\end{aligned}
$$

Substituindo $-q^{2}=Q^{2}, x=\frac{Q^{2}}{2 p \cdot q}$ e $p^{2}=m_{p}^{2}$ em (A.34):

$$
\begin{aligned}
\frac{T_{5}\left(x, Q^{2}\right)}{m_{p}^{2}} & =Q^{2} \sum_{k=1}^{\infty} \frac{2^{2 k}}{\left(Q^{2}\right)^{2 k}} \sum_{j=1}^{k-1}(-1)^{j} \frac{(2 k-j) !}{2^{j}(2 k) !} \frac{(2 k-2) !}{2^{j-1}(j-1) !(2 k-2 j-1) !} \\
& \times\left(m_{p}^{2}\right)^{j}\left(-Q^{2}\right)^{j-1}\left(\frac{Q^{2}}{2 x}\right)^{2 k-2 j-1} C_{2}^{2 k} \mathcal{A}_{2 k} \\
& +\sum_{k=1}^{\infty} \frac{2^{2 k}}{\left(Q^{2}\right)^{2 k}} \sum_{j=0}^{k-1}(-1)^{j} \frac{(2 k-j) !}{2^{j}(2 k) !} \frac{(2 k-1) !}{2^{j} j !(2 k-2 j-1) !}\left(m_{p}^{2}\right)^{j}\left(-Q^{2}\right)^{j} \\
& \times\left(\frac{Q^{2}}{2 x}\right)^{2 k-2 j-1} C_{5}^{2 k} \mathcal{A}_{2 k} \\
& =\frac{1}{Q^{2}} \sum_{k=1}^{\infty} \sum_{j=1}^{k-1} \frac{(2 k-j) !}{j !(2 k-2 j) !}\left(\frac{m_{p}^{2}}{Q^{2}}\right)^{j}\left[-\frac{2 j(2 k-2 j)}{k(2 k-1)} C_{2}^{2 k}\right. \\
& \left.+\frac{(2 k-2 j)}{k} C_{5}^{2 k}\right] \frac{\mathcal{A}_{2 k}}{x^{2 k-2 j-1}}
\end{aligned}
$$


Utilizando o binômio $\left(\begin{array}{l}n \\ k\end{array}\right)=\frac{n !}{k !(n-k) !}$ e fazendo a troca, nos índices mudos, $k=l+j$ em (A.35) tem-se:

$$
T_{5}\left(x, Q^{2}\right)=\sum_{l=1}^{\infty} \sum_{j=0}^{\infty}\left(\begin{array}{c}
2 l+j \\
j
\end{array}\right) \mu^{j+1} \frac{2 l}{l+j}\left[-2 j \frac{C_{2}^{2 l+2 j}}{2 l+2 j-1}+C_{5}^{2 l+2 j}\right] \frac{\mathcal{A}_{2 l+2 j}}{x^{2 l-1}}
$$

Lembrando que $x=\frac{1}{\omega}$ e usando as equações (2.18), (2.19), (2.35), (3.3) em (A.36) encontramos o momento de Cornwall-Norton (3.18e):

$$
\begin{aligned}
M_{5}^{(n)}\left(Q^{2}\right) & =\int_{0}^{1} x^{n-1} F_{5}^{T M C}\left(x, Q^{2}\right) d x=\frac{1}{2} \frac{1}{2 \pi i} \oint_{C} \frac{1}{\omega^{n+1}} \frac{\omega}{2 \mu} T_{5}\left(1 / \omega, Q^{2}\right) d \omega \\
& =\frac{1}{2} \sum_{l=1}^{\infty} \sum_{j=0}^{\infty}\left(\begin{array}{c}
2 l+j \\
j
\end{array}\right) \mu^{j} \frac{l}{l+j}\left[-2 j \frac{C_{2}^{2 l+2 j}}{2 l+2 j-1}+C_{5}^{2 l+2 j}\right] \mathcal{A}_{2 l+2 j} \\
& \left.=\frac{1}{2} \sum_{l=1}^{\infty} \sum_{j=0}^{\infty} \omega^{2 l-n-1} d \omega\right) \\
M_{5}^{(n)}\left(Q^{2}\right) & =\sum_{j=0}^{\infty}\left(\begin{array}{c}
n+j \\
j
\end{array}\right) \mu^{j} \frac{l}{l+j}\left[-2 j \frac{C_{2}^{2 l+2 j}}{2 l+2 j-1}+C_{5}^{2 l+2 j}\right] \mathcal{A}_{2 l+2 j} \delta_{2 l, n} \\
j & {\left[\begin{array}{c}
j \\
n+2 j
\end{array}\right] }
\end{aligned}
$$

Utilizando a transformada inversa de Mellin, (3.19) para $i=5,(3.22)$ e a substituição:

$$
\frac{C_{5}^{n+2 j} \mathcal{A}_{n+2 j}}{n+2 j}=\int_{0}^{1} d y y^{n+2 j-1} h_{5}(y)
$$

a equação (A.37) simplifica e com um pouco de álgebra chegamos em (3.30e):

$$
\begin{array}{r}
F_{5}^{T M C}\left(x, Q^{2}\right)=\frac{1}{2 \pi i} \int_{0}^{1} d y \int_{-i \infty}^{+i \infty} d n x^{-n} \sum_{j=0}^{\infty}\left(\begin{array}{c}
n+j \\
j
\end{array}\right) \mu^{j} n\left[-y^{n+2 j-2} j g_{2}(y)\right. \\
\left.+y^{n+2 j-1} \frac{h_{5}(y)}{2}\right]
\end{array}
$$




$$
\begin{aligned}
= & \left\{-\mu x^{2} \frac{\partial^{2}}{\partial x^{2}}\left[\int_{0}^{1} d y \frac{g_{2}(y)}{\left(1-\mu y^{2}\right)^{2}}\right]-\frac{x}{2} \frac{\partial}{\partial x}\left[\int_{0}^{1} d y \frac{h_{5}(y)}{y\left(1-\mu y^{2}\right)}\right]\right\} \\
& \times\left[\frac{1}{2 \pi i} \int_{-i \infty}^{+i \infty} d n\left(\frac{y}{x\left(1-\mu y^{2}\right)}\right)^{n}\right] \\
= & \left\{-\mu x^{2} \frac{\partial^{2}}{\partial x^{2}}\left[\int_{0}^{1} d y \frac{g_{2}(y)}{\left(1-\mu y^{2}\right)^{2}}\right]-\frac{x}{2} \frac{\partial}{\partial x}\left[\int_{0}^{1} d y \frac{h_{5}(y)}{y\left(1-\mu y^{2}\right)}\right]\right\} \\
& \left.\times \delta\left[\ln \left(\frac{y}{x\left(1-\mu y^{2}\right)}\right)\right]-\frac{x}{2} \frac{\partial}{\partial x}\left[\int_{0}^{1} d y \frac{h_{5}(y)}{y\left(1-\mu y^{2}\right)}\right]\right\} \\
& \left\{\delta(y-\xi) \frac{y\left(1-\mu y^{2}\right)}{1+\mu y^{2}}\right. \\
& -\mu x^{2} \frac{\partial^{2}}{\partial x^{2}}\left[\frac{\xi x_{2}(\xi)}{1-\mu^{2} \xi^{4}}\right]-\frac{x}{2} \frac{\partial}{\partial x}\left[\frac{g_{2}(y)}{1+\mu \xi^{2}}\right] \\
F_{5}^{T M C}\left(x, Q^{2}\right)= & (\mathrm{A} .39)
\end{aligned}
$$

onde foi usado que $n(n+1) x^{-n}=x^{2} \frac{\partial^{2}}{\partial x^{2}} x^{-n}, n x^{-n}=-x \frac{\partial}{\partial x} x^{-n}$, o teorema binomial (3.25), a relação (A.8), a propriedade (3.27) e a relação (3.28). 


\section{Apêndice B}

\section{Funções de Estrutura com Inversão}

\section{Termo a Termo}

Neste apêndice será realizada a derivação das funções de estrutura $F_{i}^{T M C}\left(x, Q^{2}\right)$, com $i=1, L, 3,4,5$, seguindo os procedimentos adotados para a derivação na Seção 4.1 , onde foi feito o cálculo para $F_{2}^{T M C}\left(x, Q^{2}\right)$.

\section{B.1 $F_{1}^{T M C}\left(x, Q^{2}\right)$, série em $\mu$}

Partindo da transformada inversa de Mellin, equação (3.19), para $i=1$, com o momento (3.18a), (3.20) e (3.22), tem-se:

$$
\begin{aligned}
F_{1}^{T M C}\left(x, Q^{2}\right)= & \frac{1}{2 \pi i} \int_{-i \infty}^{i \infty} d n x^{-n} M_{1}^{(n)}\left(Q^{2}\right) \\
= & \frac{1}{2 \pi i} \sum_{j=0}^{\infty} \mu^{j} \int_{-i \infty}^{i \infty} d n \int_{0}^{1} d y\left(\begin{array}{c}
n+j \\
j
\end{array}\right) x^{-n}\left[y^{n+2 j-1} \frac{f_{1}(y)}{2}\right. \\
& \left.+j y^{n+2 j-2} g_{2}(y)\right]
\end{aligned}
$$




$$
\begin{array}{r}
=\frac{1}{2 \pi i} \sum_{j=0}^{\infty} \mu^{j} \int_{-i \infty}^{i \infty} d n \int_{0}^{1} d y \frac{(n+j) !}{j ! n !} x^{-n}\left[y^{n+2 j-1} \frac{f_{1}(y)}{2}\right. \\
\left.+j y^{n+2 j-2} g_{2}(y)\right]
\end{array}
$$

Pode-se utilizar a relação:

$$
\frac{(n+j) !}{n !} x^{-n}=x(-x)^{j} \frac{\partial^{j}}{\partial x^{j}} x^{-n-1}
$$

para todo $n$ no eixo imaginário exceto a origem, substituindo (B.2) em (B.1) e com um pouco de álgebra chegamos em (4.9a):

$$
\begin{aligned}
F_{1}^{T M C}\left(x, Q^{2}\right)= & x \sum_{j=0}^{\infty} \mu^{j} \frac{(-x)^{j}}{j !} \frac{\partial^{j}}{\partial x^{j}}\left[\frac{1}{2 \pi i} \int_{-i \infty}^{i \infty} d n \int_{0}^{1} d y x^{-n-1}\left(y^{n+2 j-1} \frac{f_{1}(y)}{2}+j y^{n+2 j-2} g_{2}(y)\right)\right] \\
= & x \sum_{j=0}^{\infty} \mu^{j} \frac{(-x)^{j}}{j !} \frac{\partial^{j}}{\partial x^{j}}\left[\int_{0}^{1} d y \frac{y^{2 j-2}}{x}\left(\frac{y}{2} f_{1}(y)+j g_{2}(y)\right)\right] \\
& \times\left[\frac{1}{2 \pi i} \int_{-i \infty}^{i \infty} d n\left(\frac{y}{x}\right)^{n}\right] \\
= & x \sum_{j=0}^{\infty} \mu^{j} \frac{(-x)^{j}}{j !} \frac{\partial^{j}}{\partial x^{j}}\left[\int_{0}^{1} d y \frac{y^{2 j-2}}{x}\left(\frac{y}{2} f_{1}(y)+j g_{2}(y)\right)\right] \delta\left[\ln \left(\frac{y}{x}\right)\right] \\
= & x \sum_{j=0}^{\infty} \mu^{j} \frac{(-x)^{j}}{j !} \frac{\partial^{j}}{\partial x^{j}}\left[\int_{0}^{1} d y \frac{y^{2 j-2}}{x}\left(\frac{y}{2} f_{1}(y)+j g_{2}(y)\right) x \delta(y-x)\right] \\
F_{1}^{T M C}\left(x, Q^{2}\right)= & x \sum_{j=0}^{\infty} \mu^{j} \frac{(-x)^{j}}{j !} \frac{\partial^{j}}{\partial x^{j}}\left[x^{2 j-2}\left(\frac{x}{2} f_{1}(x)+j g_{2}(x)\right)\right]
\end{aligned}
$$

onde foi usada a propriedade (3.27) e a relação (3.28). 


\section{B.1.1 Convergência da série para $F_{1}\left(x, Q^{2}\right)$}

Na Figura (B.1) tem-se, $F_{1}$, equação (B.3), em função de $x$ e $W$ para $Q^{2}=1 G e V^{2}$, $Q^{2}=2 \mathrm{GeV}^{2}$ e $Q^{2}=5 \mathrm{GeV}^{2}$, desde o primeiro termo $(j=0)$ até o quarto termo $(j<5)$, juntamente com a equação (A.9), em todos os cálculos foi utilizada a PDF MSTW 2008 [16]. Para $Q^{2}=1 \mathrm{GeV}^{2}$ tem-se convergência com os primeiros três primeiros termos até $x \lesssim 0.45$ ou $W \gtrsim 1.45 \mathrm{GeV}$, para $Q^{2}=2 \mathrm{GeV}^{2}$ até $x \lesssim 0.65$ ou $W \gtrsim 1.4 \mathrm{GeV}$ e para $Q^{2}=5 \mathrm{GeV}^{2}$ até $x \lesssim 0.85$ ou $W \gtrsim 1.33 \mathrm{GeV}$.
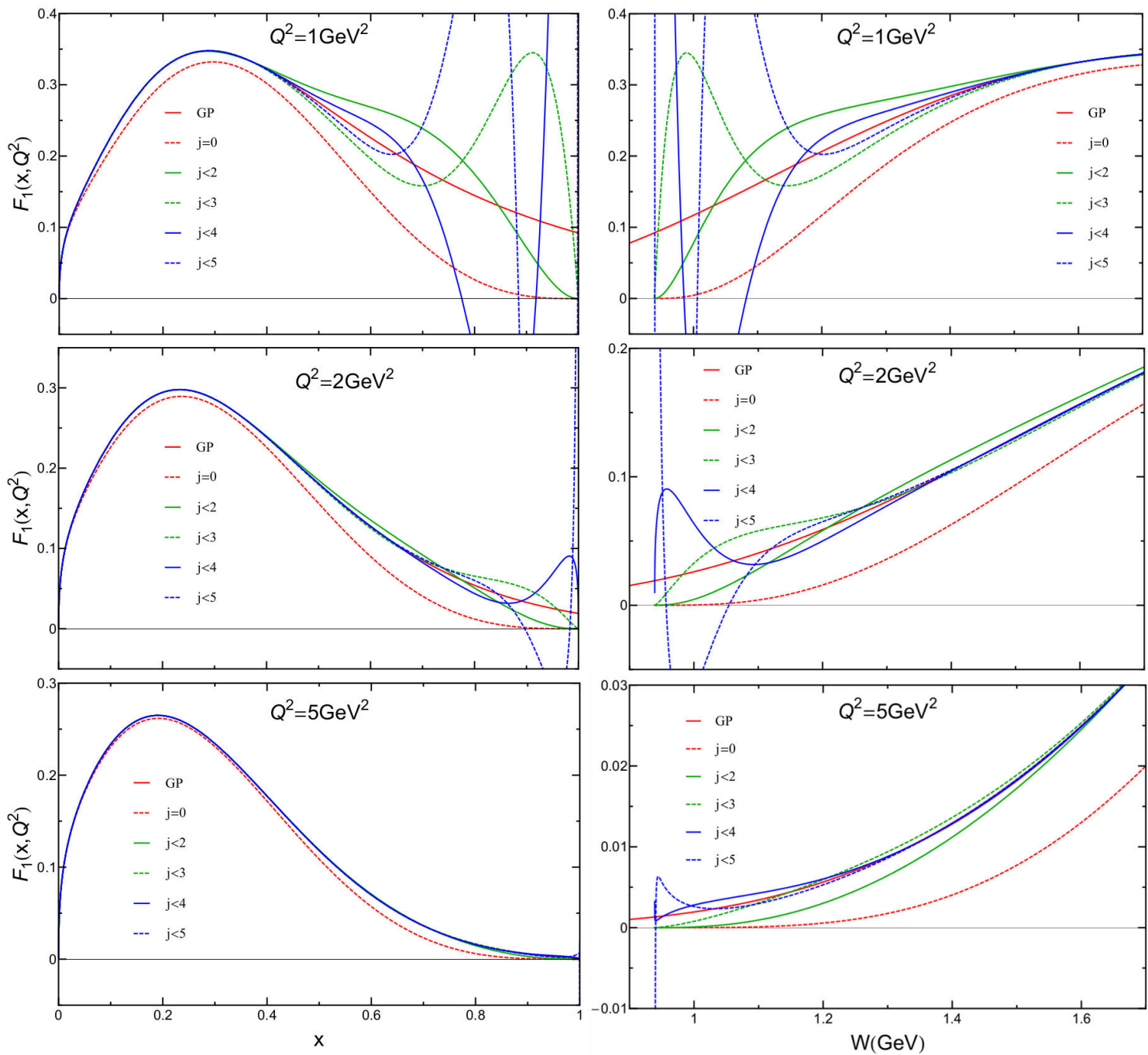

Figura B.1: TMC para $F_{1}$ em $Q^{2}=1 \mathrm{GeV}^{2}$ (acima), $Q^{2}=2 \mathrm{GeV}^{2}$ (meio) e $Q^{2}=5 \mathrm{GeV}^{2}$ (abaixo) pela equação (B.3), mostrando a convergência com o aumento de $j$, comparada com o resultado da equação (A.9), em função de $x$ (esquerda) e $W$ (direita). 
Na Figura (B.2) tem-se a razão entre a série para $F_{1}$, equação (B.3), e a TMC dada pela equação (A.9) em função de $x$ e $W$ para $Q^{2}=1 G e V^{2}, Q^{2}=2 G e V^{2}$ e $Q^{2}=5 \mathrm{GeV}^{2}$, em todos os cálculos foi utilizada a PDF MSTW 2008 [16].
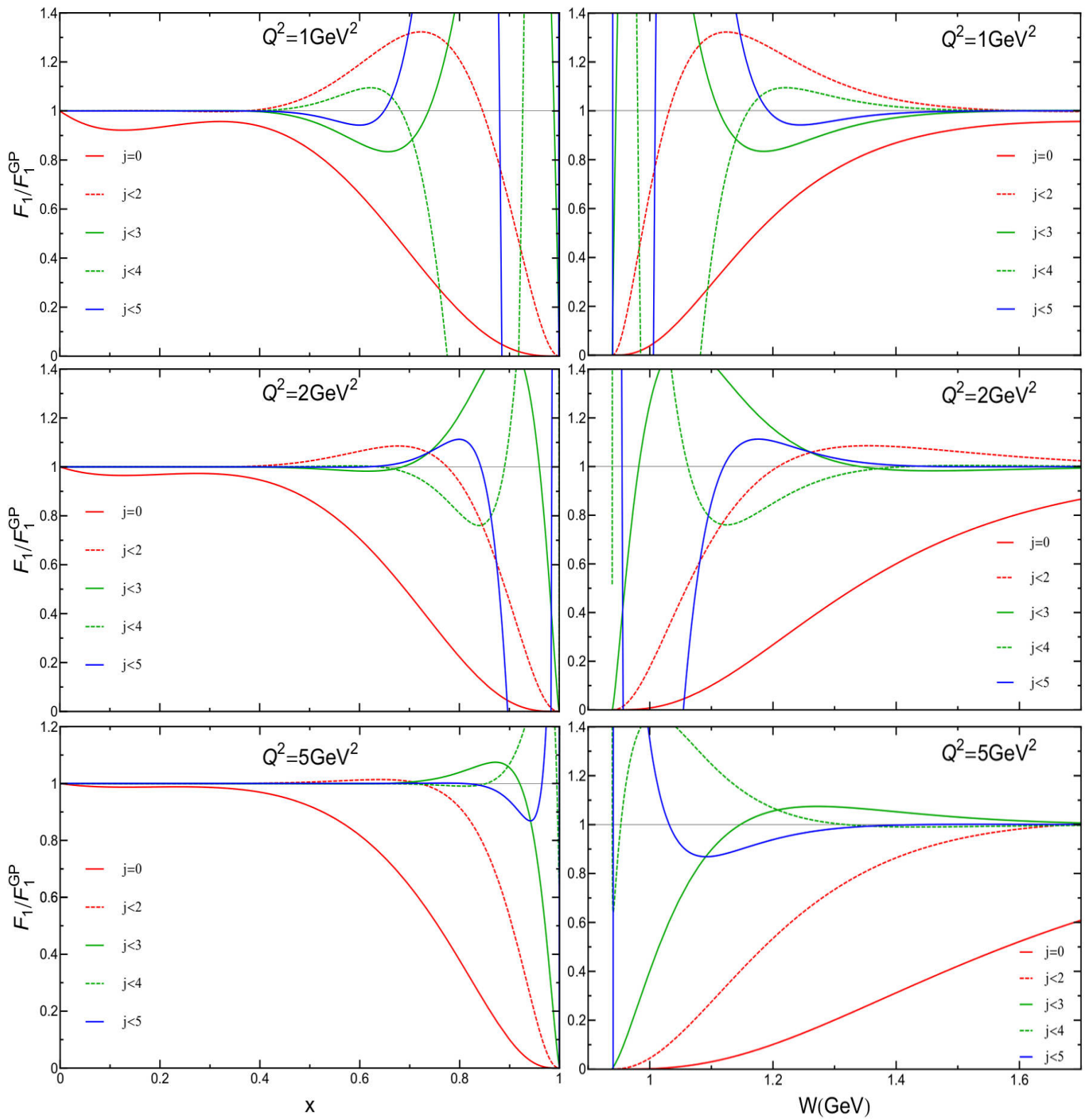

Figura B.2: Razão entre a TMC $F_{1}$, equação (B.3), em $Q^{2}=1 G e V^{2}$ (acima), $Q^{2}=2 \mathrm{GeV}^{2}$ (meio) e $Q^{2}=5 \mathrm{GeV}^{2}$ (abaixo) pela TMC dada pela equação (A.9) em função de $x$ (esquerda) e $W$ (direita), mostrando a convergência com o aumento de $j$. 


\section{B.2 $F_{L}^{T M C}\left(x, Q^{2}\right)$, série em $\mu$}

Partindo da transformada inversa de Mellin, equação (3.19), para $i=L$, com o momento (3.18b), (3.20) e (3.22), tem-se:

$$
\begin{aligned}
& F_{L}^{T M C}\left(x, Q^{2}\right)= \frac{1}{2 \pi i} \int_{-i \infty}^{i \infty} d n x^{-n+1} M_{L}^{(n)}\left(Q^{2}\right) \\
&= \frac{1}{2 \pi i} \sum_{j=0}^{\infty} \mu^{j} \int_{-i \infty}^{i \infty} d n \int_{0}^{1} d y\left(\begin{array}{c}
n+j \\
j
\end{array}\right) x^{-n+1}\left[y^{n+2 j-1}\left(f_{2}(y)-f_{1}(y)\right)\right. \\
&+x \frac{1}{2 \pi i} \sum_{j=0}^{\infty} \mu^{j} \int_{-i \infty}^{i \infty} d n \int_{0}^{1} d y \frac{(n+j) !}{j ! n !} x^{-n}\left[y^{n+2 j-1}\left(f_{2}(y)-f_{1}(y)\right)\right. \\
&\left.+4 j y^{n+2 j-2} g_{2}(y)\right]
\end{aligned}
$$

Substituindo (B.2) em (B.4) e com um pouco de álgebra chegamos em (4.9b):

$$
\begin{aligned}
F_{L}^{T M C}\left(x, Q^{2}\right)= & x^{2} \sum_{j=0}^{\infty} \mu^{j} \frac{(-x)^{j}}{j !} \frac{\partial^{j}}{\partial x^{j}}\left\{\frac { 1 } { 2 \pi i } \int _ { - i \infty } ^ { i \infty } d n \int _ { 0 } ^ { 1 } d y x ^ { - n - 1 } \left[y ^ { n + 2 j - 1 } \left(f_{2}(y)\right.\right.\right. \\
= & x^{2} \sum_{j=0}^{\infty} \mu^{j} \frac{(-x)^{j}}{j !} \frac{\partial^{j}}{\partial x^{j}}\left[\int_{0}^{1} d y \frac{y^{2 j-2}}{x}\left(y f_{2}(y)-y f_{1}(y)+4 j y_{2}(y)\right)\right] \\
& \times\left[\frac{1}{2 \pi i} \int_{-i \infty}^{i \infty} d n\left(\frac{y}{x}\right)^{n}\right] \\
= & x^{2} \sum_{j=0}^{\infty} \mu^{j} \frac{(-x)^{j}}{j !} \frac{\partial^{j}}{\partial x^{j}}\left[\int_{0}^{1} d y \frac{y^{2 j-2}}{x}\left(y f_{2}(y)-y f_{1}(y)+4 j g_{2}(y)\right)\right] \\
& \times \delta\left[\ln \left(\frac{y}{x}\right)\right]
\end{aligned}
$$




$$
\begin{aligned}
= & x^{2} \sum_{j=0}^{\infty} \mu^{j} \frac{(-x)^{j}}{j !} \frac{\partial^{j}}{\partial x^{j}}\left[\int_{0}^{1} d y \frac{y^{2 j-2}}{x}\left(y f_{2}(y)-y f_{1}(y)+4 j g_{2}(y)\right)\right] \\
& \times x \delta(y-x) \\
F_{L}^{T M C}\left(x, Q^{2}\right)= & x^{2} \sum_{j=0}^{\infty} \mu^{j} \frac{(-x)^{j}}{j !} \frac{\partial^{j}}{\partial x^{j}}\left[x^{2 j-2}\left(x f_{2}(x)-x f_{1}(x)+4 j g_{2}(x)\right)\right]
\end{aligned}
$$

onde foi usada a propriedade (3.27) e a relação (3.28).

\section{B.3 $F_{3}^{T M C}\left(x, Q^{2}\right)$, série em $\mu$}

Partindo da transformada inversa de Mellin, equação (3.19), para $i=3$, com o momento (3.18c) e (A.20), tem-se:

$$
\begin{aligned}
F_{3}^{T M C}\left(x, Q^{2}\right) & =\frac{1}{2 \pi i} \int_{-i \infty}^{i \infty} d n x^{-n} M_{3}^{(n)}\left(Q^{2}\right) \\
& =\frac{1}{2 \pi i} \sum_{j=0}^{\infty} \mu^{j} \int_{-i \infty}^{i \infty} d n \int_{0}^{1} d y\left(\begin{array}{c}
n+j \\
j
\end{array}\right) n x^{-n} y^{n+2 j-1} h_{3}(y) \\
& =\frac{1}{2 \pi i} \sum_{j=0}^{\infty} \mu^{j} \int_{-i \infty}^{i \infty} d n \int_{0}^{1} d y \frac{(n+j) !}{j !(n-1) !} x^{-n} y^{n+2 j-1} h_{3}(y)
\end{aligned}
$$

Pode-se utilizar a relação:

$$
\frac{(n+j) !}{(n-1) !} x^{-n}=(-x)^{1+j} \frac{\partial^{1+j}}{\partial x^{1+j}} x^{-n}
$$

para todo $n$ no eixo imaginário exceto a origem, substituindo (B.7) em (B.6) e com um pouco de álgebra chegamos em (4.9c):

$$
F_{3}^{T M C}\left(x, Q^{2}\right)=\sum_{j=0}^{\infty} \mu^{j} \frac{(-x)^{1+j}}{j !} \frac{\partial^{1+j}}{\partial x^{1+j}}\left[\frac{1}{2 \pi i} \int_{-i \infty}^{i \infty} d n \int_{0}^{1} d y x^{-n} y^{n+2 j-1} h_{3}(y)\right]
$$




$$
\begin{aligned}
& =\sum_{j=0}^{\infty} \mu^{j} \frac{(-x)^{1+j}}{j !} \frac{\partial^{1+j}}{\partial x^{1+j}}\left[\int_{0}^{1} d y y^{2 j-1} h_{3}(y)\right]\left[\frac{1}{2 \pi i} \int_{-i \infty}^{i \infty} d n\left(\frac{y}{x}\right)^{n}\right] \\
& =\sum_{j=0}^{\infty} \mu^{j} \frac{(-x)^{1+j}}{j !} \frac{\partial^{1+j}}{\partial x^{1+j}}\left[\int_{0}^{1} d y y^{2 j-1} h_{3}(y)\right] \delta\left[\ln \left(\frac{y}{x}\right)\right] \\
& =\sum_{j=0}^{\infty} \mu^{j} \frac{(-x)^{1+j}}{j !} \frac{\partial^{1+j}}{\partial x^{1+j}}\left[\int_{0}^{1} d y y^{2 j-1} h_{3}(y) x \delta(y-x)\right] \\
F_{3}^{T M C}\left(x, Q^{2}\right) & =\sum_{j=0}^{\infty} \mu^{j} \frac{(-x)^{1+j}}{j !} \frac{\partial^{1+j}}{\partial x^{1+j}}\left[x^{2 j} h_{3}(x)\right]
\end{aligned}
$$

onde foi usada a propriedade (3.27) e a relação (3.28).

\section{B.3.1 Convergência da série para $F_{3}\left(x, Q^{2}\right)$}

Na Figura (B.3) tem-se, $F_{3}$, equação (B.8), em função de $x$ e $W$ para $Q^{2}=1 G e V^{2}$, $Q^{2}=2 \mathrm{GeV}^{2}$ e $Q^{2}=5 \mathrm{GeV}^{2}$, desde o primeiro termo $(j=0)$ até o quarto termo $(j<5)$, juntamente com a equação (A.21), em todos os cálculos foi utilizada a PDF MSTW 2008 [16]. Para $Q^{2}=1 \mathrm{GeV}^{2}$ tem-se convergência com os primeiros três primeiros termos até $x \lesssim 0.5$ ou $W \gtrsim 1.37 \mathrm{GeV}$, para $Q^{2}=2 \mathrm{GeV}^{2}$ até $x \lesssim 0.7$ ou $W \gtrsim 1.32 \mathrm{GeV}$ e para $Q^{2}=5 \mathrm{GeV}^{2}$ até $x \lesssim 0.75$ ou $W \gtrsim 1.6 \mathrm{GeV}$.

Na Figura (B.4) tem-se a razão entre a série para $F_{3}$, equação (B.8), e a TMC dada por equação (A.21) em função de $x$ e $W$ para $Q^{2}=1 G e V^{2}, Q^{2}=2 G e V^{2}$ e $Q^{2}=5 \mathrm{GeV}^{2}$, em todos os cálculos foi utilizada a PDF MSTW 2008 [16]. 

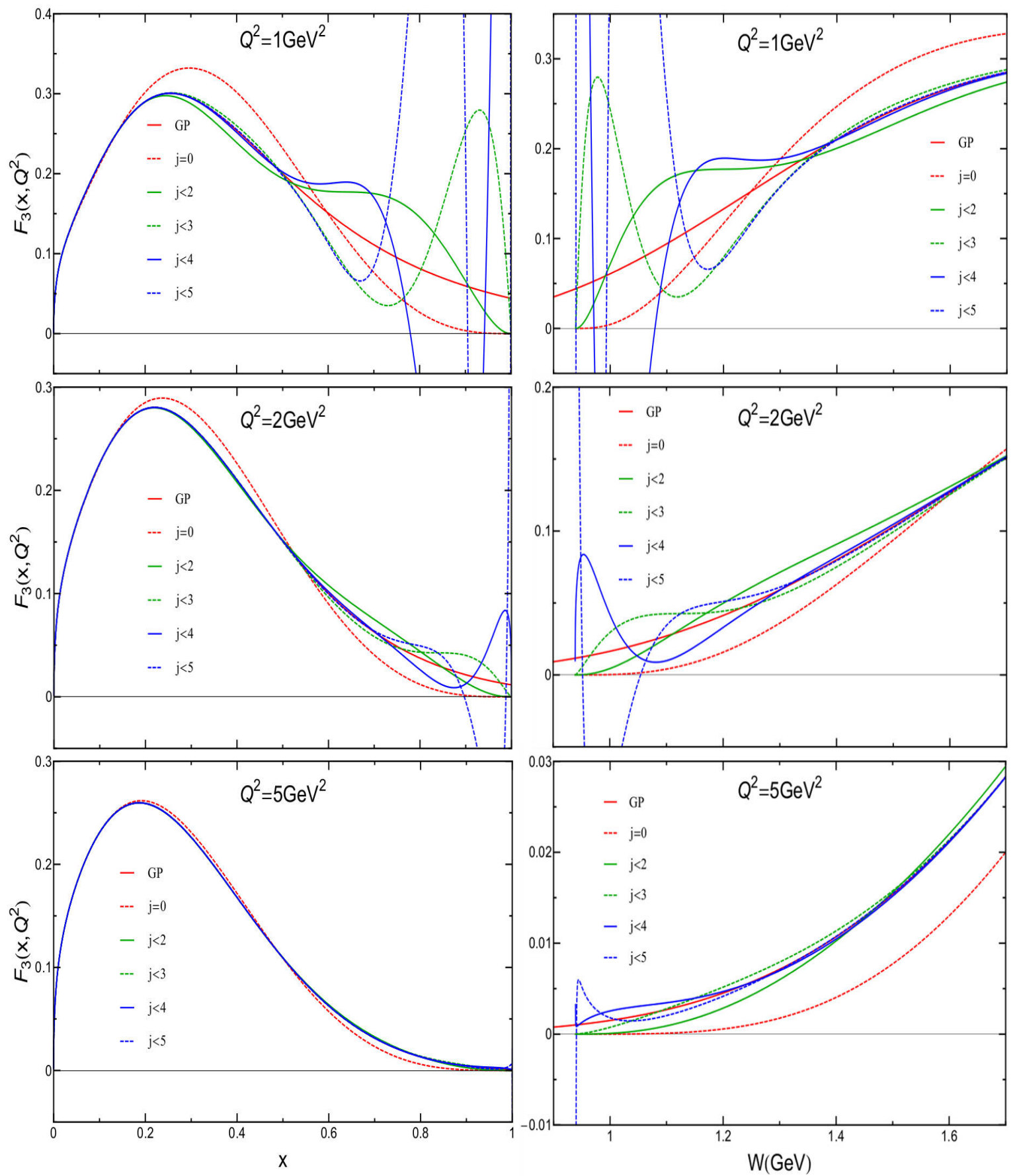

Figura B.3: TMC para $F_{3}$ em $Q^{2}=1 \mathrm{GeV}^{2}$ (acima), $Q^{2}=2 \mathrm{GeV}^{2}$ (meio) e $Q^{2}=5 \mathrm{GeV}^{2}$ (abaixo) pela equação (B.8), mostrando a convergência com o aumento de $j$, comparada com o resultado da equação (A.21), em função de $x$ (esquerda) e $W$ (direita). 

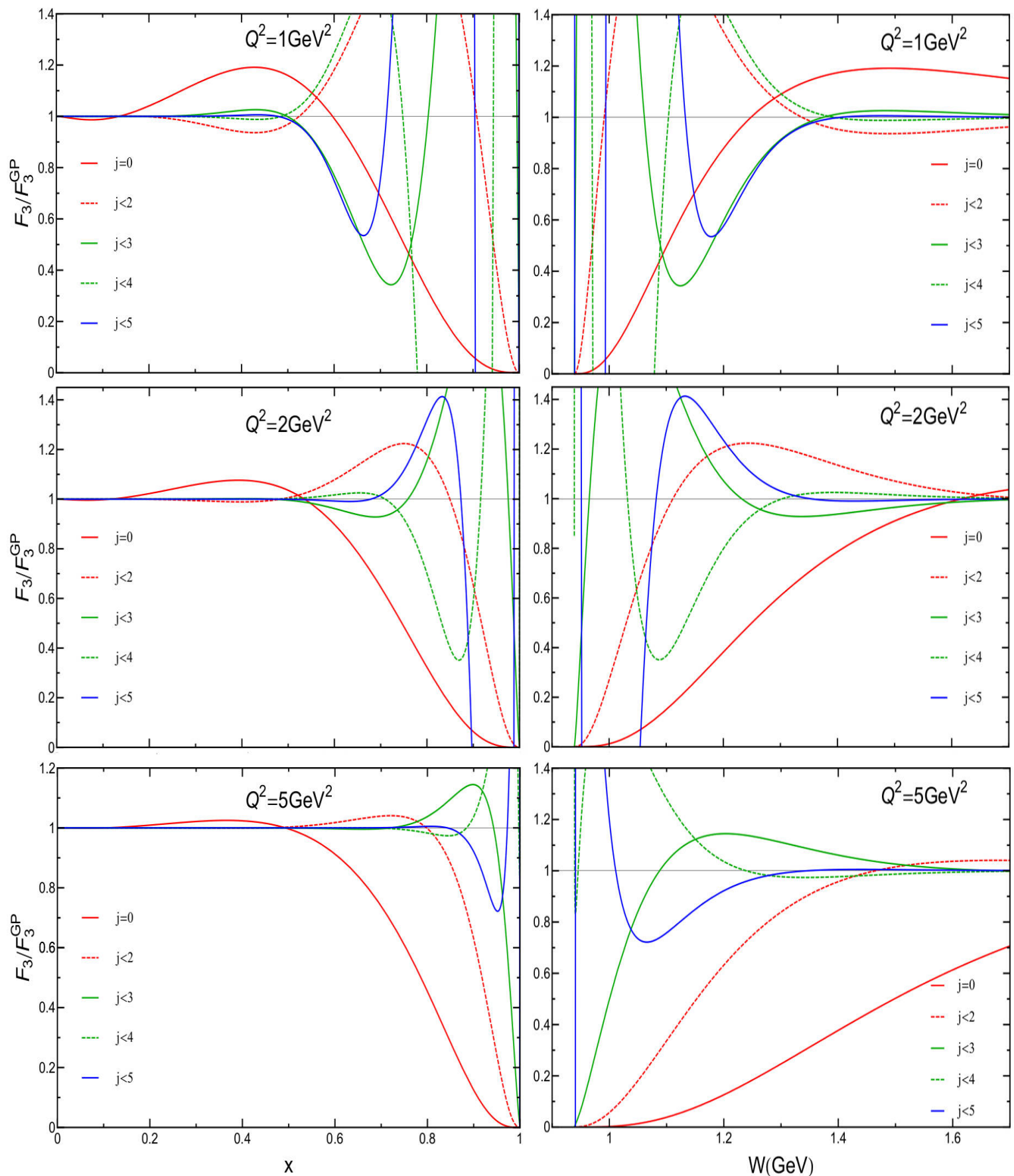

Figura B.4: Razão entre a TMC $F_{3}$, equação (B.8), em $Q^{2}=1 \mathrm{GeV}^{2}$ (acima), $Q^{2}=2 \mathrm{GeV}^{2}$ (meio) e $Q^{2}=5 \mathrm{GeV}^{2}$ (abaixo) pela TMC dada pela equação (A.21) em função de $x$ (esquerda) e $W$ (direita), mostrando a convergência com o aumento de $j$. 


\section{B.4 $F_{4}^{T M C}\left(x, Q^{2}\right)$, série em $\mu$}

Partindo da transformada inversa de Mellin, equação (3.19), para $i=4$, com o momento (3.18d), (3.20), (3.22) e (A.29), tem-se:

$$
\begin{aligned}
F_{4}^{T M C}\left(x, Q^{2}\right) & =\frac{1}{2 \pi i} \int_{-i \infty}^{i \infty} d n x^{-n} M_{4}^{(n)}\left(Q^{2}\right) \\
& =\frac{1}{2 \pi i} \sum_{j=0}^{\infty} \mu^{j} \int_{-i \infty}^{i \infty} d n \int_{0}^{1} d y\left(\begin{array}{c}
n+j \\
j
\end{array}\right) x^{-n}\left[j(j-1) y^{n+2 j-2} g_{2}(y)\right. \\
& \left.=\frac{1}{2 \pi i} \sum_{j=0}^{\infty} \mu^{j} \int_{-i \infty}^{i \infty} d n \int_{0}^{1} d y \frac{(n+2 j-1}{j ! n !} \frac{f_{4}(y)}{4}-j y^{n+2 j-2} g_{5}(y)\right] \\
& \left.+y^{n+2 j-1} \frac{f_{4}(y)}{4}-j y^{n+2 j-2} g_{5}(y)\right]
\end{aligned}
$$

Substituindo (B.2) em (B.9) e com um pouco de álgebra chegamos em (4.9d):

$$
\begin{aligned}
F_{4}^{T M C}\left(x, Q^{2}\right)= & x \sum_{j=0}^{\infty} \mu^{j} \frac{(-x)^{j}}{j !} \frac{\partial^{j}}{\partial x^{j}}\left[\frac { 1 } { 2 \pi i } \int _ { - i \infty } ^ { i \infty } d n \int _ { 0 } ^ { 1 } d y x ^ { - n - 1 } \left(j(j-1) y^{n+2 j-2} g_{2}(y)\right.\right. \\
& \left.\left.+y^{n+2 j-1} \frac{f_{4}(y)}{4}-j y^{n+2 j-2} g_{5}(y)\right)\right] \\
= & x \sum_{j=0}^{\infty} \mu^{j} \frac{(-x)^{j}}{j !} \frac{\partial^{j}}{\partial x^{j}}\left[\int _ { 0 } ^ { 1 } d y \frac { y ^ { 2 j - 2 } } { x } \left(j(j-1) g_{2}(y)+\frac{y}{4} f_{4}(y)\right.\right. \\
= & x \sum_{j=0}^{\infty} \mu^{j} \frac{(-x)^{j}}{j !} \frac{\partial^{j}}{\partial x^{j}}\left[\int _ { 0 } ^ { 1 } d y \frac { y ^ { 2 j - 2 } } { x } \left(j(j-1) g_{2}(y)+\frac{y}{4} f_{4}(y)\right.\right. \\
\left.-j g_{5}(y)\right) & \left.d n\left(\frac{1}{x}\right)^{n}\right] \\
-i \infty i \infty & \left.\left(\frac{y}{x}\right)\right]
\end{aligned}
$$




$$
\begin{array}{r}
=x \sum_{j=0}^{\infty} \mu^{j} \frac{(-x)^{j}}{j !} \frac{\partial^{j}}{\partial x^{j}}\left[\int _ { 0 } ^ { 1 } d y \frac { y ^ { 2 j - 2 } } { x } \left(j(j-1) g_{2}(y)+\frac{y}{4} f_{4}(y)\right.\right. \\
\left.\left.-j g_{5}(y)\right) x \delta(y-x)\right] \\
F_{4}^{T M C}\left(x, Q^{2}\right)=x \sum_{j=0}^{\infty} \mu^{j} \frac{(-x)^{j}}{j !} \frac{\partial^{j}}{\partial x^{j}}\left[\begin{array}{r}
x^{2 j-2}(j-1) g_{2}(x) \\
\left.+\frac{x}{4} f_{4}(x)-j g_{5}(x)\right)
\end{array}\right)
\end{array}
$$

onde foi usada a propriedade (3.27) e a relação (3.28).

\section{B.5 $F_{5}^{T M C}\left(x, Q^{2}\right)$, série em $\mu$}

Partindo da transformada inversa de Mellin, equação (3.19), para $i=5$, com o momento (3.18e), (3.22) e (A.38), tem-se:

$$
\begin{aligned}
F_{5}^{T M C}\left(x, Q^{2}\right)= & \frac{1}{2 \pi i} \int_{-i \infty}^{i \infty} d n x^{-n} M_{5}^{(n)}\left(Q^{2}\right) \\
= & \frac{1}{2 \pi i} \sum_{j=0}^{\infty} \mu^{j} \int_{-i \infty}^{i \infty} d n \int_{0}^{1} d y\left(\begin{array}{c}
n+j \\
j
\end{array}\right) n x^{-n}\left[-j y^{n+2 j-2} g_{2}(y)\right. \\
& \left.+y^{n+2 j-1} \frac{h_{5}(y)}{2}\right] \\
= & \frac{1}{2 \pi i} \sum_{j=0}^{\infty} \mu^{j} \int_{-i \infty}^{i \infty} d n \int_{0}^{1} d y \frac{(n+j) !}{j !(n-1) !} x^{-n}\left[\begin{array}{c}
-j y^{n+2 j-2} g_{2}(y) \\
\left.+y^{n+2 j-1} \frac{h_{5}(y)}{2}\right](\mathrm{B} .1
\end{array}\right.
\end{aligned}
$$


Substituindo (B.7) em (B.11) e com um pouco de álgebra chegamos em (4.9e):

$$
\begin{aligned}
& F_{5}^{T M C}\left(x, Q^{2}\right)=\sum_{j=0}^{\infty} \mu^{j} \frac{(-x)^{1+j}}{j !} \frac{\partial^{1+j}}{\partial x^{1+j}}\left[\frac { 1 } { 2 \pi i } \int _ { - i \infty } ^ { i \infty } d n \int _ { 0 } ^ { 1 } d y x ^ { - n } \left(-j y^{n+2 j-2} g_{2}(y)\right.\right. \\
& \left.\left.+y^{n+2 j-1} \frac{h_{5}(y)}{2}\right)\right] \\
& =\sum_{j=0}^{\infty} \mu^{j} \frac{(-x)^{1+j}}{j !} \frac{\partial^{1+j}}{\partial x^{1+j}}\left[\int_{0}^{1} d y y^{2 j-2}\left(-j g_{2}(y)+\frac{y}{2} h_{5}(y)\right)\right] \\
& \times\left[\frac{1}{2 \pi i} \int_{-i \infty}^{i \infty} d n\left(\frac{y}{x}\right)^{n}\right] \\
& =\sum_{j=0}^{\infty} \mu^{j} \frac{(-x)^{1+j}}{j !} \frac{\partial^{1+j}}{\partial x^{1+j}}\left[\int_{0}^{1} d y y^{2 j-2}\left(-j g_{2}(y)+\frac{y}{2} h_{5}(y)\right)\right] \\
& \times \delta\left[\ln \left(\frac{y}{x}\right)\right] \\
& =\sum_{j=0}^{\infty} \mu^{j} \frac{(-x)^{1+j}}{j !} \frac{\partial^{1+j}}{\partial x^{1+j}}\left[\int_{0}^{1} d y y^{2 j-2}\left(-j g_{2}(y)+\frac{y}{2} h_{5}(y)\right)\right] \\
& \times x \delta(y-x) \\
& F_{5}^{T M C}\left(x, Q^{2}\right)=\sum_{j=0}^{\infty} \mu^{j} \frac{(-x)^{1+j}}{j !} \frac{\partial^{1+j}}{\partial x^{1+j}}\left[x^{2 j-1}\left(-j g_{2}(x)+\frac{x}{2} h_{5}(x)\right)\right]
\end{aligned}
$$

onde foi usada a propriedade (3.27) e a relação (3.28). 


\section{Bibliografia}

[1] J. D. Bjorken, Phys. Rev. 179, 1547 (1969).

[2] E. D. Bloom, D. H. Coward, H. C. DeStaebler, J. Drees, G. Miller, L. W. Mo, R. E. Taylor and M. Breidenbach et al., Phys. Rev. Lett. 23, 930 (1969); M. Breidenbach, J. I. Friedman, H. W. Kendall, E. D. Bloom, D. H. Coward, H. C. DeStaebler, J. Drees and L. W. Mo et al., Phys. Rev. Lett. 23, 935 (1969).

[3] R. P. Feynman, Conf. Proc. C 690905, 237 (1969).

[4] R. P. Feynman, Photon-hadron interactions, Reading 1972, 282p.

[5] M. Gell-Mann, Phys. Lett. 8, 214 (1964).

[6] G. Zweig, CERN-TH-412 (1964); G. Zweig, PRINT-64-170 (1964).

[7] G. Miller, E. D. Bloom, G. Buschhorn, D. H. Coward, H. C. DeStaebler, J. Drees, C. L. Jordan and L. W. Mo et al., Phys. Rev. D 5, 528 (1972).

[8] L. D. Faddeev and V. N. Popov, Phys. Lett. B 25, 29 (1967); G. 't Hooft, Nucl. Phys. B 33, 173 (1971); H. Fritzsch, M. Gell-Mann and H. Leutwyler, Phys. Lett. B 47, 365 (1973).

[9] C. Berger et al. [Pluto Collaboration], Phys. Lett. B 76, 243 (1978).

[10] J. Beringer et al. [Particle Data Group Collaboration], Phys. Rev. D 86, 010001 (2012). 
[11] H1 Collaboration: I. Abt et al. Nucl. Phys. B407 515 (1993); S. Aid et al., Nucl. Phys. B470 3 (1996); C. Adloff et al., ibid. B497 3 (1997). C. Adloff et al., Eur. Phys. J. C13 609 (2000) [hep-ex/9908059]; Eur. Phys. J. C19 269 (2001) [hepex/0012052]; Eur. Phys. J. C21 33 (2001) [hep-ex/0012053]. C. Adloff et al. Phys. Lett. B520 183 (2001);

[12] ZEUS Collaboration: M. Derrick et al. Phys. Lett. B316, 412 (1993); M. Derrick et al., Z. Phys. C72, 399 (1996); J. Breitweg et al. Eur. Phys. J. C7 609 (1999) S. Chekanov et al., Eur. Phys. J. C21 443 (2001) [hep-ex/0105090]; A.M. Cooper-Sarkar, Proceedings of International Europhysics Conference on HEP 2001, Budapest [hep-ph/0110386].

[13] V. N. Gribov and L. N. Lipatov, Sov. J. Nucl. Phys. 15 (1972) 438 [Yad. Fiz. 15 (1972) 781]; G. Altarelli and G. Parisi, Nucl. Phys. B 126, 298 (1977); Y. L. Dokshitzer, Sov. Phys. JETP 46, 641 (1977) [Zh. Eksp. Teor. Fiz. 73, $1216(1977)]$.

[14] J. Pumplin, D. R. Stump, J. Huston, H. L. Lai, P. M. Nadolsky and W. K. Tung, JHEP 0207, 012 (2002) [hep-ph/0201195].

[15] P. Jimenez-Delgado and E. Reya, Phys. Rev. D 79, 074023 (2009) [arXiv:0810.4274 [hep-ph]].

[16] A. D. Martin, W. J. Stirling, R. S. Thorne and G. Watt, Eur. Phys. J. C 63, 189 (2009).

[17] S. Alekhin, J. Blumlein, S. Klein and S. Moch, arXiv:0908.3128 [hep-ph].

[18] J. F. Owens, A. Accardi and W. Melnitchouk, arXiv:1212.1702 [hep-ph].

[19] A. Cooper-Sarkar, arXiv:1107.5170 [hep-ph].

[20] L. V. Gribov. E. M. Levin e M. G. Ryskin, Phys. Rep. 100, 1 (1983). 
[21] J. E. Cole, J. -W. Qiu and U. -k. Yang, AIP Conf. Proc. 792, 121 (2005) [hepex/0508003]; W. Melnitchouk, AIP Conf. Proc. 1261, 85 (2010) [arXiv:1006.4134 [nucl-th]]; L. T. Brady, A. Accardi, W. Melnitchouk and J. F. Owens, JHEP 1206, 019 (2012) [arXiv:1110.5398 [hep-ph]].

[22] S. Kuhlmann, J. Huston, J. Morfin, F. I. Olness, J. Pumplin, J. F. Owens, W. K. Tung and J. J. Whitmore, Phys. Lett. B 476, 291 (2000) [hep$\mathrm{ph} / 9912283]$.

[23] K. -P. O. Diener, S. Dittmaier and W. Hollik, Phys. Rev. D 72, 093002 (2005) [hep-ph/0509084]; A. D. Martin, R. G. Roberts, W. J. Stirling and R. S. Thorne, Eur. Phys. J. C 39, 155 (2005) [hep-ph/0411040].

[24] R. M. Barnett, Phys. Rev. Lett. 36, 1163 (1976); S. Kretzer and I. Schienbein, Phys. Rev. D 58, 094035 (1998) [hep-ph/9805233].

[25] O. Nachtmann, Nucl. Phys. B 63, 237 (1973).

[26] H. Georgi and H. D. Politzer, Phys. Rev. D 14, 1829 (1976).

[27] I. Schienbein et al., J. Phys. G 35, 053101 (2008) [arXiv:0709.1775 [hep-ph]].

[28] K. G. Wilson, Phys. Rev. 179, 1499 (1969).

[29] R. K. Ellis, R. Petronzio and G. Parisi, Phys. Lett. B 64, 97 (1976); R. Barbieri, J. R. Ellis, M. K. Gaillard and G. G. Ross, Phys. Lett. B 64, 171 (1976); R. Barbieri, J. R. Ellis, M. K. Gaillard and G. G. Ross, Nucl. Phys. B 117, 50 (1976); D. J. Gross, S. B. Treiman and F. A. Wilczek, Phys. Rev. D 15, 2486 (1977); W. R. Frazer and J. F. Gunion, Phys. Rev. Lett. 45, 1138 (1980).

[30] K. Bitar, P. W. Johnson and W. K. Tung, Phys. Lett. B 83, 114 (1979); P. W. Johnson and W. K. Tung, PRINT-79-1018 (ILLINOIS-TECH).

[31] F. Halzen and A. D. Martin, Quarks And Leptons: An Introductory Course In Modern Particle Physics, New York, Usa: Wiley (1984) 396p. 
[32] N. F. Mott, Proc. R. Soc. A124 365 (1929).

[33] R. Hofstadter, Ann. Rev. Nucl. Part. Sci. 7, 231 (1957).

[34] O. Stern, Nature 132, 103 (1950).

[35] M. N. Rosenbluth, Phys. Rev. 79, 615 (1950).

[36] E. Leader e E. Predazzi, An Introduction to gauge theories and modern particle physics. Vol. 1: Electroweak interactions, the new particles and the parton model, Camb. Monogr. Part. Phys. Nucl. Phys. Cosmol. 3, 1 (1996).

[37] J. Dudek, R. Ent, R. Essig, K. Kumar, C. Meyer, R. McKeown, Z. E. Meziani and G. A. Miller et al., Eur. Phys. J. A 48, 187 (2012) [arXiv:1208.1244 [hep-ex]].

[38] R. P. Feynman, Phys. Rev. Lett. 23, 1415 (1969).

[39] W. K. H. Panofsky, SLAC-PUB-0502.

[40] J. D. Bjorken, In *Bjorken, J.D.: A collection of summary talks in high energy physics* 41-51, SLAC-PUB-0571, (1969);

J. D. Bjorken and E. A. Paschos, Phys. Rev. 185, 1975 (1969).

[41] C. G. Callan, Jr. and D. J. Gross, Phys. Rev. Lett. 22, 156 (1969).

[42] R. Jackiw and G. Preparata, Phys. Rev. Lett. 22, 975 (1969); S. L. Adler and W. -K. Tung, Phys. Rev. Lett. 22, 978 (1969).

[43] D. J. Griffiths, Introduction To Elementary Particles, NEW YORK, USA: WILEY (1987) 392p.

[44] C. H. Llewellyn Smith, Phys. Rept. 3, 261 (1972).

[45] R. D. Field and R. P. Feynman, Phys. Rev. D 15, 2590 (1977).

[46] P. Amaudruz et al. [New Muon Collaboration], Phys. Rev. Lett. 66, 2712 (1991);

M. Arneodo et al. [New Muon Collaboration], Phys. Rev. D 50, 1 (1994). 
[47] A. Baldit et al. [NA51 Collaboration], Phys. Lett. B 332, 244 (1994).

[48] E. M. Henley and G. A. Miller, Phys. Lett. B 251, 453 (1990); A. I. Signal, A. W. Schreiber and A. W. Thomas, Mod. Phys. Lett. A 6, 271 (1991); W. Y. P. Hwang, J. Speth and G. E. Brown, Z. Phys. A 339, 383 (1991); W. Melnitchouk, A. W. Thomas and A. I. Signal, Z. Phys. A 340, 85 (1991); S. Kumano and J. T. Londergan, Phys. Rev. D 44, 717 (1991); E. J. Eichten, I. Hinchliffe and C. Quigg, Phys. Rev. D 45, 2269 (1992); V. R. Zoller, Z. Phys. C 54, 425 (1992); A. Szczurek and J. Speth, Nucl. Phys. A 555, 249 (1993).

[49] A. W. Thomas and W. Melnitchouk, In *Shimoda/Tokyo 1993, New frontiers in nuclear physics* 41-106; W. Koepf, L. L. Frankfurt and M. Strikman, Phys. Rev. D 53, 2586 (1996) [hep-ph/9507218].

[50] J. Ashman et al. [European Muon Collaboration], Phys. Lett. B 206, 364 (1988); J. Ashman et al. [European Muon Collaboration], Nucl. Phys. B 328, 1 (1989).

[51] A. Chodos, R. L. Jaffe, K. Johnson, C. B. Thorn and V. F. Weisskopf, Phys. Rev. D 9, 3471 (1974).

[52] R. L. Jaffe and A. Manohar, Nucl. Phys. B 337, 509 (1990); S. D. Bass and A. W. Thomas, J. Phys. G 19, 925 (1993); M. Anselmino, A. Efremov and E. Leader, Phys. Rept. 261, 1 (1995) [Erratum-ibid. 281, 399 (1997)] [hep$\mathrm{ph} / 9501369]$.

[53] B. Adeva et al. [Spin Muon Collaboration], Phys. Lett. B 302, 533 (1993); P. L. Anthony et al. [E142 Collaboration], Phys. Rev. Lett. 71, 959 (1993); D. Adams et al. [Spin Muon (SMC) Collaboration], Phys. Lett. B 329, 399 (1994) [hep-ph/9404270]; K. Abe et al. [E143 Collaboration], Phys. Rev. Lett. 75, 25 (1995); D. Adams et al. [Spin Muon Collaboration], Phys. Lett. B 357, 248 (1995); K. Abe et al. [E143 Collaboration], Phys. Rev. Lett. 74, 346 (1995). 
[54] A. Airapetian et al. [HERMES Collaboration], Phys. Rev. D 75, 012007 (2007) [hep-ex/0609039]; E. S. Ageev et al. [Compass Collaboration], Phys. Lett. B 647, 330 (2007) [hep-ex/0701014].

[55] A. W. Thomas, Phys. Rev. Lett. 101, 102003 (2008) [arXiv:0803.2775 [hep-ph]].

[56] E. Comay, Prog. Phys. 1, 75 (2011) [arXiv:1011.1610 [physics.gen-ph]].

[57] A. W. Thomas, Prog. Part. Nucl. Phys. 61, 219 (2008) [arXiv:0805.4437 [hep$\mathrm{ph}]$.

[58] W. Zimmermann, Annals Phys. 77, 570 (1973) [Lect. Notes Phys. 558, 278 $(2000)]$.

[59] G. C. Wick, Phys. Rev. 80, 268 (1950).

[60] T. Muta, Foundations of quantum chromodynamics. Third edition, World Sci. Lect. Notes Phys. 78, 1 (2010).

[61] S. Weinberg, The quantum theory of fields. Vol. 2: Modern applications, Cambridge, UK: Univ. Pr. (1996).

[62] W. Greiner and J. Reinhardt, Field quantization, Berlin, Germany: Springer (1996).

[63] S. G. Krantz, Handbook of Complex Variables, Boston, MA: Birkhuser (1999).

[64] N. H. Christ, B. Hasslacher and A. H. Mueller, Phys. Rev. D 6, 3543 (1972);

R. L. Jaffe, MIT-CTP-1261.

[65] R. A. Brandt and G. Preparata, Nucl. Phys. B 27, 541 (1972).

[66] D. J. Gross and S. B. Treiman, Phys. Rev. D 4, 1059 (1971).

[67] H. Georgi and H. D. Politzer, Phys. Rev. D 9, 416 (1974); D. J. Gross and F. Wilczek, Phys. Rev. D 9, 980 (1974); H. D. Politzer, Phys. Rept. 14, 129 (1974); A. Peterman, Phys. Rept. 53, 157 (1979). 
[68] A. J. G. Hey and J. E. Mandula, Phys. Rev. D 5, 2610 (1972).

[69] F. J. Yndurain, The theory of quark and gluon interactions, Berlin, Germany: Springer (2006) $474 \mathrm{p}$.

[70] J. M. Cornwall and R. E. Norton, Phys. Rev. 177, 2584 (1969); I. L. Solovtsov and D. V. Shirkov, Theor. Math. Phys. 120, 1220 (1999) [Teor. Mat. Fiz. 120, 482 (1999)] [hep-ph/9909305].

[71] A. Accardi, M. E. Christy, C. E. Keppel, P. Monaghan, W. Melnitchouk, J. G. Morfin and J. F. Owens, Phys. Rev. D 81, 034016 (2010) [arXiv:0911.2254 [hep-ph]].

[72] P. M. Nadolsky, H. -L. Lai, Q. -H. Cao, J. Huston, J. Pumplin, D. Stump, W. K. Tung and C. -P. Yuan, Phys. Rev. D 78, 013004 (2008) [arXiv:0802.0007 [hep-ph]]; S. Alekhin, J. Blumlein, S. Klein and S. Moch, Phys. Rev. D 81, 014032 (2010) [arXiv:0908.2766 [hep-ph]]; R. D. Ball, V. Bertone, F. Cerutti, L. Del Debbio, S. Forte, A. Guffanti, J. I. Latorre and J. Rojo et al., Nucl. Phys. B 849, 296 (2011) [arXiv:1101.1300 [hep-ph]].

[73] Y. Liang et al. [Jefferson Lab Hall C E94-110 Collaboration], nucl-ex/0410027; S. P. Malace et al. [Jefferson Lab E00-115 Collaboration], Phys. Rev. C 80, 035207 (2009) [arXiv:0905.2374 [nucl-ex]]; P. Monaghan, A. Accardi, M. E. Christy, C. E. Keppel, W. Melnitchouk and L. Zhu, arXiv:1209.4542 [nucl-ex].

[74] JLab Experiment E12-06-113; Jlab Experiment E12-10-103.

[75] S. Kretzer and M. H. Reno, Phys. Rev. D 69, 034002 (2004) [hep-ph/0307023].

[76] J. Blumlein, Prog. Part. Nucl. Phys. 69, 28 (2013) [arXiv:1208.6087 [hep-ph]].

[77] H. Mellin, Acta Soc. Sci. Fennica 21, 1 (1896); H. Mellin, Acta Math. 25, 139 (1902); H. J. Weber and G. B. Arfken, Essential Mathematical Methods for Physicists, Academic Press (2003) 932 p. 
[78] F. M. Steffens, M. D. Brown, W. Melnitchouk and S. Sanches, Phys. Rev. C 86, 065208 (2012) [arXiv:1210.4398 [hep-ph]].

[79] M. Tzanov et al. [NuTeV Collaboration], Phys. Rev. D 74, 012008 (2006) [hepex/0509010].

[80] S. Kretzer, H. L. Lai, F. I. Olness and W. K. Tung, Phys. Rev. D 69, 114005 (2004) [hep-ph/0307022].

[81] W. Melnitchouk, Nucl. Phys. A 782, 126 (2007).

[82] A. J. Buras, Rev. Mod. Phys. 52, 199 (1980).

[83] S. Wandzura, Nucl. Phys. B 122, 412 (1977).

[84] O. Nachtmann, Nucl. Phys. B 78, 455 (1974); V. Baluni and E. Eichten, Phys. Rev. D 14, 3045 (1976); V. Baluni and E. Eichten, Phys. Rev. Lett. 37, 1181 (1976); Y. Kitadono, K. Sasaki, T. Ueda and T. Uematsu, Phys. Rev. D 77, 054019 (2008) [arXiv:0801.0937 [hep-ph]].

[85] F. M. Steffens and W. Melnitchouk, Phys. Rev. C 73, 055202 (2006) [nuclth/0603014].

[86] E. D. Bloom and F. J. Gilman, Phys. Rev. Lett. 25, 1140 (1970); W. Melnitchouk, Phys. Rev. Lett. 86, 35 (2001) [Erratum-ibid. 93, 199901 (2004)] [hepph/0106073].

[87] A. De Rujula, H. Georgi and H. D. Politzer, Phys. Rev. D 15, 2495 (1977); A. De Rujula, H. Georgi and H. D. Politzer, Annals Phys. 103, 315 (1977).

[88] I. Niculescu et al., Phys. Rev. Lett. 85, 1186 (2000).

[89] R. K. Ellis, W. Furmanski and R. Petronzio, Nucl. Phys. B 212, 29 (1983). 
[90] M. A. G. Aivazis, F. I. Olness and W. -K. Tung, Phys. Rev. D 50, 3085 (1994) [hep-ph/9312318]; S. Kretzer and M. H. Reno, Phys. Rev. D 66, 113007 (2002) [hep-ph/0208187];

[91] A. Accardi and J. W. Qiu, JHEP 0807, 090 (2008) [arXiv:0805.1496 [hep-ph]].

[92] U. D'Alesio, E. Leader and F. Murgia, Phys. Rev. D 81, 036010 (2010) [arXiv:0909.5650 [hep-ph]].

[93] F. M. Steffens, AIP Conf. Proc. 1369, 189 (2011) [arXiv:1103.5710 [hep-ph]].

[94] R. J. Holt, AIP Conf. Proc. 1369, 5 (2011).

[95] U. -K. Yang and A. Bodek, Phys. Rev. Lett. 82, 2467 (1999) [hep-ph/9809480]; L. W. Whitlow, E. M. Riordan, S. Dasu, S. Rock and A. Bodek, Phys. Lett. B 282, 475 (1992); L. L. Frankfurt and M. I. Strikman, Phys. Rept. 160, 235 (1988).

[96] W. Melnitchouk and A. W. Thomas, Phys. Lett. B 377, 11 (1996) [nuclth/9602038].

[97] A. Bodek, M. Breidenbach, D. L. Dubin, J. E. Elias, J. I. Friedman, H. W. Kendall, J. S. Poucher and E. M. Riordan et al., Phys. Rev. D 20, 1471 (1979).

[98] D. Boer, M. Diehl, R. Milner, R. Venugopalan, W. Vogelsang, D. Kaplan, H. Montgomery and S. Vigdor et al., arXiv:1108.1713 [nucl-th]. 